\title{
Linear Dynamic Space Mapping Approach for Large-signal Statistical Modeling of

\author{
Microwave Devices
}

\author{
by \\ Kui Bo, B. Eng., \\ A thesis submitted to the Faculty of Graduate Studies and Research \\ in partial fulfillment of the requirement for the \\ degree of Master of Applied Science
}

Ottawa-Carleton Institute for Electrical and Computer Engineering Department of Electronics Carleton University

Ottawa, Ontario K1S 5B6

Canada

(C) Copyright August 2007, Kui Bo 


$\begin{array}{ll}\begin{array}{l}\text { Library and } \\ \text { Archives Canada }\end{array} & \begin{array}{l}\text { Bibliothèque et } \\ \text { Archives Canada }\end{array} \\ \begin{array}{l}\text { Published Heritage } \\ \text { Branch }\end{array} & \begin{array}{l}\text { Direction du } \\ \text { Patrimoine de l'édition }\end{array} \\ \begin{array}{l}\text { 395 Wellington Street } \\ \text { Ottawa ON K1A ON4 }\end{array} & \begin{array}{l}\text { 395, rue Wellington } \\ \text { Ottawa ON K1A ON4 } \\ \text { Canada }\end{array}\end{array}$

Your file Votre référence ISBN: 978-0-494-33638-0 Our file Notre référence ISBN: 978-0-494-33638-0

NOTICE:

The author has granted a nonexclusive license allowing Library and Archives Canada to reproduce, publish, archive, preserve, conserve, communicate to the public by telecommunication or on the Internet, loan, distribute and sell theses worldwide, for commercial or noncommercial purposes, in microform, paper, electronic and/or any other formats.

The author retains copyright ownership and moral rights in this thesis. Neither the thesis nor substantial extracts from it may be printed or otherwise reproduced without the author's permission.
AVIS:

L'auteur a accordé une licence non exclusive permettant à la Bibliothèque et Archives Canada de reproduire, publier, archiver, sauvegarder, conserver, transmettre au public par télécommunication ou par l'Internet, prêter, distribuer et vendre des thèses partout dans le monde, à des fins commerciales ou autres, sur support microforme, papier, électronique et/ou autres formats.

L'auteur conserve la propriété du droit d'auteur et des droits moraux qui protège cette thèse. $\mathrm{Ni}$ la thèse ni des extraits substantiels de celle-ci ne doivent être imprimés ou autrement reproduits sans son autorisation.
In compliance with the Canadian

Privacy Act some supporting forms may have been removed from this thesis.

While these forms may be included in the document page count, their removal does not represent any loss of content from the thesis.
Conformément à la loi canadienne sur la protection de la vie privée, quelques formulaires secondaires ont été enlevés de cette thèse.

Bien que ces formulaires aient inclus dans la pagination, il n'y aura aucun contenu manquant.

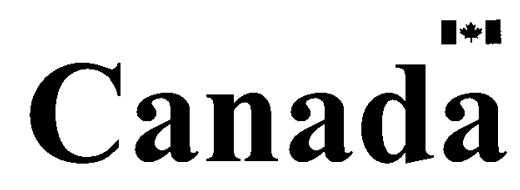




\begin{abstract}
This thesis presents a novel technique for large-signal statistical modeling of nonlinear microwave devices. A new statistical space mapping concept is introduced that can expand a large-signal nominal model into a large-signal statistical model. The nominal model is an accurate large-signal model developed from one complete large-signal measurement and it describes the nominal performance of the device population. The mapping contains the statistical parameters estimated by fitting the DC and biasdependent S-parameter data of the device population. In this way, the nominal model mainly represents the large-signal nonlinear behavior of the device population while the random variations around the nominal model are represented by the space mapping functions. It helps to efficiently develop large-signal statistical models while reducing the expense of otherwise massive large-signal measurements for many devices. Examples of MESFET and HEMT statistical modeling demonstrate that the technique can approximate the large-signal statistical characteristics using only one set of large-signal data. The use of such statistical model in amplifier yield design further demonstrates the capability of the technique in capturing the large-signal statistical properties.
\end{abstract}




\section{Acknowledgements}

First of all, I would like to express my profound gratitude to my thesis supervisor Professor Qi-Jun Zhang for his professional guidance, continued assistance, invaluable inspiration, encouragement, and patience throughout the research work and the preparation of this thesis. His moral support and continuous guidance enabled me to complete my work successfully. I am highly indebted to him for providing the chances to complete myself not only into a researcher with technical, but also a responsible person with active personalities. And I also want to say thanks to my colleague, Lei Zhang for her prompt answer for my questions and suggestion for practical applications.

My deep appreciation is to my colleague and best friend Yi Cao for his generous and helpful discussions. Humayun Kabir, Shang Wan, and Xin Zhang in my group are thanked for the help, friendship, and laugh with the entire collective working.

Many special thanks to Blazenka Power, Peggy Piccolo, Lorena Duncan, Nagui Mikhail, Jacques Lemieux, Scott Bruce and all other staff and faculty for providing the excellent lab facilities and friendly environment for study and research.

And, as always, the heartfelt thanks are given to my parents for their love and sweet smiles always being there for me.

Finally, I want to dedicate this work to my grand parents for their endless love, support, and encouragement for me to live my dream all the time. 


\section{Contents}

1 Introduction 1

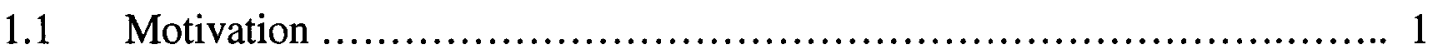

1.2 Thesis Objective.................................................... 3

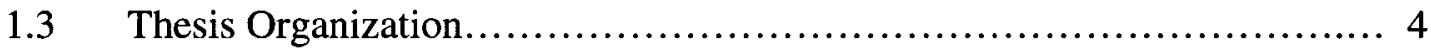

2 Literature Review 6

2.1 Artificial Neural Networks for Nonlinear Device Modeling................... 7

2.1.1 Neural Network Applications in Microwave/RF Circuit Design.......... 7

2.1.2 Neural Network Structures........................................... 9

2.2 Knowledge-Based Nonlinear Dynamic Modeling........................ 14

2.2.1 Knowledge-Based Nonlinear Dynamic Modeling................... 15

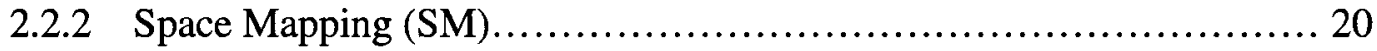

2.2.3 Difference Method (DM) for Nonlinear Modeling..................... 21

2.2.4 Neual-Space Mapping (Neural-SM) ............................ 24

2.3 Brief Overview of Conventional Statistical Modeling Techniques......... 30

2.4 Conclusions........................................................... 31

3 Proposed Linear Dynamic Space Mapping Technique 33

3.1 Linear Dynamic Space Mapping Technique.......................... 34

3.2 Detail in Proposed Linear Dynamic Space-mapped Technique.............. 40

3.3 Procedures of the Modeling......................................... 43 
3.4 Objective of the Optimization for Statistical Linear Dynamic Space Mapping Model 46

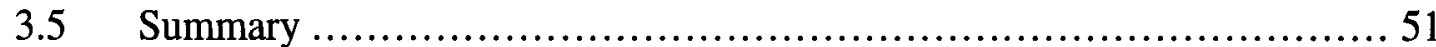

4 Application Examples of Linear Dynamic Space Mapping Technique 53

4.1 MESFET Statistical Modeling.............................................. 53

4.2 HEMT Statistical Modeling.............................................. 65

4.3 Statistical Model Used in Amplifier Simulation........................ 77

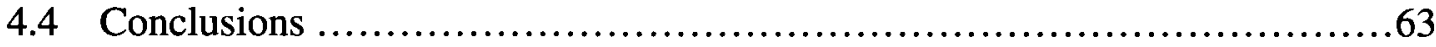

5 Conclusions and Future Research $\quad 85$

$\begin{array}{llr}6 & \text { Bibliography } & 87\end{array}$ 


\section{List of Figures}

Figure 2.1: Multilayer Perceptrons (MLP) neural network structure. Typically, a MLP network consists of an input layer, one or more hidden layers, and an output layer................................................................ 11

Figure 2.2: Commonly used nonlinear activation functions of hidden neurons in MLP network........................................................... 12

Figure 2.3: Knowledge Based Neural Network (KBNN) structure. Typically a KBNN

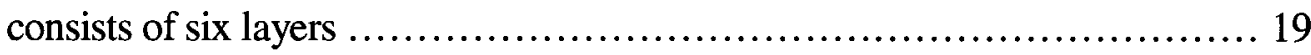

Figure 2.4: Structure of space mapping model.................................. 21

Figure 2.5: Knowledge-based structure for nonlinear modeling utilizing DM

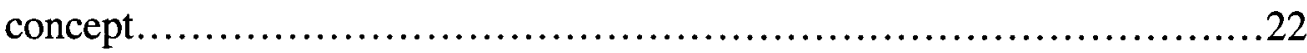

Figure 2.6: Circuit representation of the DNN model..............................23

Figure 2.7: General 2-port Neuro-SM nonlinear model...........................27

Figure 3.1: General large-signal statistical modeling (a) Device population. (b) Simulated model population.

Figure 3.2: Large-signal statistical modeling approach: Statistical space-mapped model with linear dynamic mapping...................................... 39

Figure 3.3: Two-port statistical space-mapped model...............................41

Figure 3.4: Flow chart of linear dynamic space mapping for large-signal statistical modeling.... .45

Figure 4.1: Figure 4.1 Comparison of mean value $(\mu)$ and standard deviation $(\sigma)$ of real and imaginary parts of S-parameters between 250 MESFET devices (-) and 
250 proposed statistical linear dynamic space mapping models (x). Error of mean is $0.62 \%$ and error of standard deviation is $67.44 \% \ldots \ldots \ldots \ldots \ldots \ldots . . .58$

Figure 4.2: Figure 4.2 Comparison of ECDF of DC and S-parameters in real and imaginary parts between 250 MESFET devices (dash line) and 250 proposed statistical linear dynamic space mapping models (solid line). Overall error is $6.47 \%$ 59

Figure 4.3: Power Gain Comparison between 100 statistical models and 100 devices: (a) Mean and standard deviation (b) Distribution (c) ECDF - device in dash line and model in solid line

Figure 4.4: TOI Comparison between 100 statistical models and 100 devices: (a) Mean and standard deviation (b) Distribution (c) ECDF - device in dash line and model in solid line

Figure 4.5: PAE Comparison between 100 statistical models and 100 devices: (a) Mean and standard deviation (b) Distribution (c) ECDF - device in dash line and model in solid line

Figure 4.6: HEMT structure in Medici............................................ 67

Figure 4.7: Comparison of mean value $(\mu)$ and standard deviation ( $\sigma)$ of real and imaginary parts of S-parameters between 250 MESFET devices (-) and 250 proposed statistical linear dynamic space mapping models (x). Error of mean is $1.64 \%$ and error of standard deviation is $51.02 \%$ 69

Figure 4.8: Comparison of ECDF of DC and S-parameters in real and imaginary parts between 250 MESFET devices (dash line) and 250 proposed statistical linear 
dynamic space mapping models (solid line). Overall error is

$6.60 \%$

Figure 4.9: Fundamental harmonic comparisons between 100 statistical models and 100 devices: (a) Mean and standard deviation device in (-) and model in (x). (b) Distribution (c) ECDF - device in dash line and model in solid line.

Figure 4.10: Second harmonic comparisons between 100 statistical models and 100 devices: (a) Mean and standard deviation device in (-) and model in (x). (b) Distribution (c) ECDF - device in dash line and model in solid line.

Figure 4.11: Third harmonic comparisons between 100 statistical models and 100 devices: (a) Mean and standard deviation device in (-) and model in (x). (b) Distribution (c) ECDF - device in dash line and model in solid line.

Figure 4.12: Example of output power (fundamental to third harmonics) vs. input power of Monte-Carlo simulations with 100 devices using (a) original ADS MESFET and (b) proposed statistical space-mapped model. 78

Figure 4.13: Example of output current of Monte-Carlo simulations with 100 devices using (a) original ADS MESFET and (b) proposed statistical space-mapped model. 79

Figure 4.14: Three-stage amplifier circuit 82

Figure 4.15: Gain comparison of 1000 amplifier circuits using (a) original ADS MESFET and (b) proposed statistical space-mapped model. The distribution of the 
amplifier responses using proposed statistical space-mapped model matches that of the original ADS results well, confirming the proposed method..... 83 


\section{List of Tables}

Table 4.1: Mean/Nominal Values and Equivalent parameter Standard Deviation Change for MESFET Example 54

Table 4.2: Mean of Statistical Parameters Before and After Parameter

Regeneration 55

Table 4.3: FET Standard Deviation of Statistical Parameters Before and After Parameter

Regeneration. 56

Table 4.4: Correlation Coefficients of Statistical Parameters Before Parameter

Regeneration. 56

Table 4.5: Correlation Coefficients of Statistical Parameters After Parameter

Regeneration. 56

Table 4.6: Comparison Between the Device Data and the Statistical Linear Dynamic Mapping Model. 64

Table 4.7: Mean Values and Physical/Geometrical Parameters Change for HEMT

Example 67

Table 4.8: Mean of Statistical Parameters Before and After Parameter

Regeneration. 68

Table 4.9: Standard Deviation of Statistical Parameters Before and After Parameter

Regeneration. 68

Table 4.10: Correlation Coefficients of Statistical Parameters Before Parameter

Regeneration 68 
Table 4.11: Correlation Coefficients of Statistical Parameters After Parameter

Regeneration..................................................... 68

Table 4.12: Comparison Between the Device Data and the Statistical Linear Dynamic

Mapping Model................................................. 76

Table 4.13: Means and Standard Deviations of the Statistical Space Mapping

Parameters.......................................................... 80

Table 4.14: Correlation Coefficients of the Statistical Space Mapping Parameters....... 81 


\section{Chapter 1}

\section{Introduction}

\subsection{Motivation}

In recent years, radio frequency $(\mathrm{RF})$ and microwave circuits are experiencing rapid growth and development on both the research and industrial sides leading to reduce device dimensions and increase complexities of circuit functionality. At the same time, statistical models of microwave devices and integrated circuits are essential to take account for the process parameter variations during circuit analysis and design. Hence, methods for developing accurate statistical models are getting attention in the research community now.

Most of the recent and practical microwave nonlinear statistical modeling approaches are based on DC and bias-dependent S-parameter measurements [1]-[4]. Usually, tedious measurements have to be performed on many devices in order to obtain the statistical information. Corresponding to each device, a set of seriously measured DC and S-parameter data is converted to the corresponding parameters in the equivalent circuit through a parameter extraction procedure. The statistical properties of the equivalent circuit parameters are then examined, thus the estimates of the means, the standard deviations, and the correlation coefficients are calculated. 
Finally, statistical models based on some multivariate or heuristic techniques capable of recreating these means, standard deviations, and correlations can be developed [5].

In the past several years, nonlinear device modeling directly using large-signal measurement data has gained recognition due to the increasing need of accuracy in characterizing nonlinear device behaviors. The advanced computer-aided design (CAD) tools now can handle extensive design, analysis, and optimization for modeling those high frequency nonlinear behavior; however, direct large-signal statistical modeling still remains prohibitive because complete nonlinear largesignal measurement for many modern complex circuits and devices is too expensive and time consuming.

This thesis investigates the potential of using large-signal data for nonlinear statistical modeling. A novel large-signal statistical modeling method is proposed that combines one large-signal nominal model and a linear dynamic space mapping functions that is evolved from Neuro-space Mapping network [6]. The large-signal nominal model is developed using one complete set of large-signal data. It describes the nominal performance of a given device population. A new statistical space mapping concept is introduced to account for the large-signal statistical properties. The mapping contains statistical parameters estimated by fitting many DC and bias dependent S-parameter data of the given device population. In this way, the nonlinear behavior of the large-signal model is described in a combination of the nominal model and the space mapping functions, which presents the random variations around the nominal model. Based on the assumption that the parameter 
variations of the given device population is usually a small percentage of their nominal values, such that a set of simple mapping functions could be extracted from small-signal data to approximate the large-signal statistical variations. This technique is demonstrated through modeling nonlinear MESFET and HEMT devices in DC and S-parameters, harmonic balance, and two-tone harmonic balance, and finally the use in the amplifier yield analysis.

\subsection{Thesis Objective}

The main objective of this thesis is to develop a novel nonlinear statistical modeling technique that not only enables the accurate and fast statistical modeling of small-signals, but also makes the technique applicable for large-signal modeling.

In this thesis, the proposed linear dynamic space mapping technique [7] for largesignal statistical modeling is presented and with evidence to prove the objectives:

1) First of all, a new statistical modeling technique is proposed in large-signal of the nonlinear devices and circuits. The technique combines a large-signal nominal model and linear dynamic space mapping functions to carefully characterize the large-signal statistical variation around the nominal. Compared to the existing and commonly used brute force large-signal statistical modeling techniques for nonlinear devices, which requires the large-signal measurements for many devices, the proposed technique requires only one set of large-signal measurement combined with small-signal information for the rest of the devices, which are much easier to measure. 
2) Based on the proposed mapping technique, a robust algorithm that facilitates efficient statistical modeling in large-signal of nonlinear devices is developed. The algorithm makes the statistical modeling more systematic and accurate by constructing a nominal model that represents the main nonlinear behavior of the whole statistical population while the random variation properties around the nominal model are represented by the space mapping [6] functions.

3) The proposed technique enables the statistical modeling concept to be applied to the nonlinear device modeling in large-signal circuit simulation. In this proposed technique, the voltage and current signals between the existing device model (namely, the nominal model) and the actual devices behavior (namely, the fine model) are mapped by a linear mapped function, such that the mapped models accurately match the behavior of the actual devices.

\subsection{Thesis Organization}

The thesis is organized as follows:

In Chapter 2, the Artificial Neural Networks (ANNs) modeling of nonlinear devices is first briefly reviewed because the proposed technique is evolved from a most up-to-date neural network modeling technique. Following is the review of different knowledge-based nonlinear dynamic modeling techniques, which are directly related to the proposed technique. In particular, the difference and NeuroSpace Mapping (Neuro-SM) techniques are reviewed in detail. Finally, a brief summary of conventional statistical modeling for nonlinear devices is presented. 
Chapter 3 introduces the new technique; Linear Dynamic Space Mapping technique for statistical modeling in large-signal of nonlinear devices, which followed the existing knowledge-based modeling techniques. It inherits all the advantages from the existing large-signal modeling methods and further directs the knowledge-based modeling techniques into the filed of statistical modeling in largesignal. With the knowledge-based structure, linear dynamic space mapping technique can be applied to various microwave modeling cases and to achieve accurate models.

In Chapter 4, the applications of linear dynamic space mapping for nonlinear statistical modeling are presented. A general structure for a nonlinear general 2-port network with input mapping neural network realized by controlled sources is proposed. Based on the new algorithm for DC, small-signal, and large-signal simulation and optimization, examples are applied. Detailed examples of GaAs MESFET and HEMT modeling are provided, which proves the proposed technique is an efficient and accurate approach in developing large-signal statistical model for nonlinear microwave devices. Also the use of the model in harmonic balance simulation and power amplifier demonstrates that the linear dynamic space mapping is a practical and systematic method that allows us to apply.

Finally, in Chapter 5, conclusions and suggestions for future research are discussed. 


\section{Chapter 2}

\section{Literature Review}

Nonlinear device modeling has always been the most important activity in RF and microwave CAD. Presently most popular nonlinear modeling approaches are based on the equivalent circuit models [6] - [8]. We all know that numbers of equivalent circuit models have been developed in the past because no single equivalent circuit model can represent all kinds of nonlinear device behaviors. In the modern society, new RF and microwave devices are constantly evolving appearing in smaller size and more functions, and existing nonlinear models are not able to fully represent the behaviors in the new devices. Developing new equivalent circuit models becomes RF and microwave scientist's major task; however, often a timeconsuming trial-and-error process is used for formulating new equivalent circuit topology and for creating formulas of nonlinear elements.

In recent years, several advanced neural networks based methods have been published to address the nonlinear modeling issues [9] [10]. These methods depend on the device data for neural network training, which leads to the model development without the use of device equivalent circuits or device equations.

In this chapter, we describe a recent method in details. This method combines the neural network learning with the existing device equivalent circuit models, 
which are used as prior knowledge. Thus, we can develop more reliable models with less data, and at the same time improve the extrapolation capability of the model.

Because of the rapid change in modern RF and microwave integrated circuit design, the statistical modeling of nonlinear devices gained the focus now. Many statistical modeling methods have been developed based on DC and small-signal parameters; however, very few methods could be applied to the statistical modeling in nonlinear large-signal. The development of the proposed technique is just based on the existing neural network and statistical modeling technique for the statistical modeling in large-signal.

\subsection{Artificial Neural Networks for Nonlinear}

\section{Device Modeling}

\subsubsection{Neural Network Applications in Microwave/RF Circuit Design}

Compared to the existing modeling techniques, neural network models are much faster than the detailed physical/EM models [9] [11], more accurate than the polynomial and empirical models [12], capable of more dimensions than the lookup table models 
[13], and are much easier to develop when new device/technology is invented [14]. Once the structure is constructed, these neural network models can be used in places of physical/EM models of active and passive components, which involved intensive computation. Also the neural networks can accelerate the microwave circuit design cycle.

The fast learning ability of neural networks is very useful because often it is not easy to create the analytical model for a new invented device. ANN has been successfully used in a variety of applications such as modeling and optimization of high-speed VLSI interconnects [15], microstrip interconnects [16], vias [17], spiral inductors [18], coplanar waveguide (CPW) circuit components [19], filter [20], mixers [21], antennas [22], embedded resistors [23] [24], microwave FETs and amplifiers [9][25], CMOS and HBTs [26] [27], HEMT devices [28], EM-optimization [29], yield optimization [9] and circuit synthesis [30] [31], etc. These achievements have set up the foundation of neural modeling technique in both device and circuit level of microwave applications.

In order to meet the demands of high manufacturability and fast design cycles, we need an efficient statistical design technique. Statistical analysis and yield optimization, which take into account of the manufacturing tolerance, model uncertainties, and variation in the process parameters, etc., are widely accepted as indispensable components of the circuit design methodology [32]. Detailed physical/ EM models of active/passive components can be an important step towards a design, but the models are computationally intensive. Since significant advances have been made in the exploitation of artificial neural networks as an unconventional alternative to modeling and design tasks in microwave/RF CAD, now the future use of neural network for statistical modeling becomes the goal of the researchers. 


\subsubsection{Neural Network Structures}

Over the years, a variety of neural network structures have been applied to RF and microwave modeling. A neural network is specifically defined by the activation functions in the hidden layer and the neurons are connected with each other. In this section, we describe several important structures of neural networks, including multilayer perceptrons (MLP) and knowledge-based neural network (KBNN).

Multilayer Perceptron (MLP) network is a class of feedforward neural networks that have been widely used in the microwave applications due to its simplicity of the structure and capability of modeling nonlinear functions [33]. In an MLP neural network, the neurons are grouped into layers. The first layer and the last layers are called input and output layers, respectively. The remaining middle layers are called hidden layers. Typically, an MLP neural network consists of an input layer, one or more hidden layers, and an output layer, as shown in Figure 2.1. Let $L$ be the total number of layers. The first layer is the input layer, the $L^{\text {th }}$ layer is the output layer, and layers 2 to $L-1$ are hidden layers. Let $N_{l}$ be the number of neurons in the $l^{\text {th }}$ layer is $l=1, \ldots, L$. Let $w_{i j}^{l}$ represent the weight of the link between the $j^{\text {th }}$ neuron of $(l-1)^{t h}$ hidden layer and the $i^{t h}$ neuron of $l^{\text {th }}$ hidden layer. There is an additional weight parameter for each neuron $w_{i 0}^{l}$ representing the bias for the $i^{\text {th }}$ neuron of the $l^{\text {th }}$ layer. We define a weight vector $\boldsymbol{w}$ containing all the trainable parameters of MLP as

$$
\boldsymbol{w}=\left[\begin{array}{lllll}
w_{10}^{2} & w_{11}^{2} & w_{12}^{2} & \ldots & w_{N_{L} N_{L-1}}^{L}
\end{array}\right]^{T}
$$


Let $x_{i}$ represent the $i^{\text {th }}$ input parameter to the MLP. Given the MLP input $x_{i} i=$ $1, \ldots, N_{1}$, The feedforward computation is a process starting from the input layer and proceeding the following hidden layers to produce the layer output

$$
\begin{gathered}
z_{i}^{1}=x_{i}, \quad i=1, \ldots, N_{1} \\
z_{i}^{l}=\sigma\left(\sum_{j=1}^{N_{l-1}} w_{i j}^{l} z_{i}^{l-1}+w_{i 0}^{l}\right), \quad i=1, \ldots, N_{l}, \quad l=2, \ldots, L-1
\end{gathered}
$$

where $\sigma$ is the activation function of hidden neurons and $z_{i}^{l}$ is the output of $i^{\text {th }}$ neuron of $l^{\text {th }}$ hidden layer. The most commonly used form of $\sigma$ is the logistic sigmoid function given by

$$
\sigma(\gamma)=\frac{1}{\left(1+e^{-\gamma}\right)}
$$

It is a smooth switch from 0 to 1 as $\gamma$ varies from negative infinity to positive infinity. Other possible candidates for $\sigma$ could be arctangent function

$$
\sigma(\gamma)=\left(\frac{2}{\pi}\right) \arctan (\gamma)
$$

or hyperbolic tangent function

$$
\sigma(\gamma)=\frac{\left(e^{\gamma}-e^{-\gamma}\right)}{\left(e^{\gamma}+e^{-\gamma}\right)}
$$

Fig. 2.2 shows the plots of these nonlinear activation functions for hidden neurons. It can be noted that all these activation functions are smooth switch functions that are bounded, continuous, monotonic and continuously differentiable.

Finally, The MLP output is calculated from the output layer (linear layer)

$$
y_{k}=\sum_{i=1}^{N_{L-1}} w_{k i} z_{i}^{L-1}+w_{k 0}^{L}, \quad k=1, \ldots, N_{L}
$$




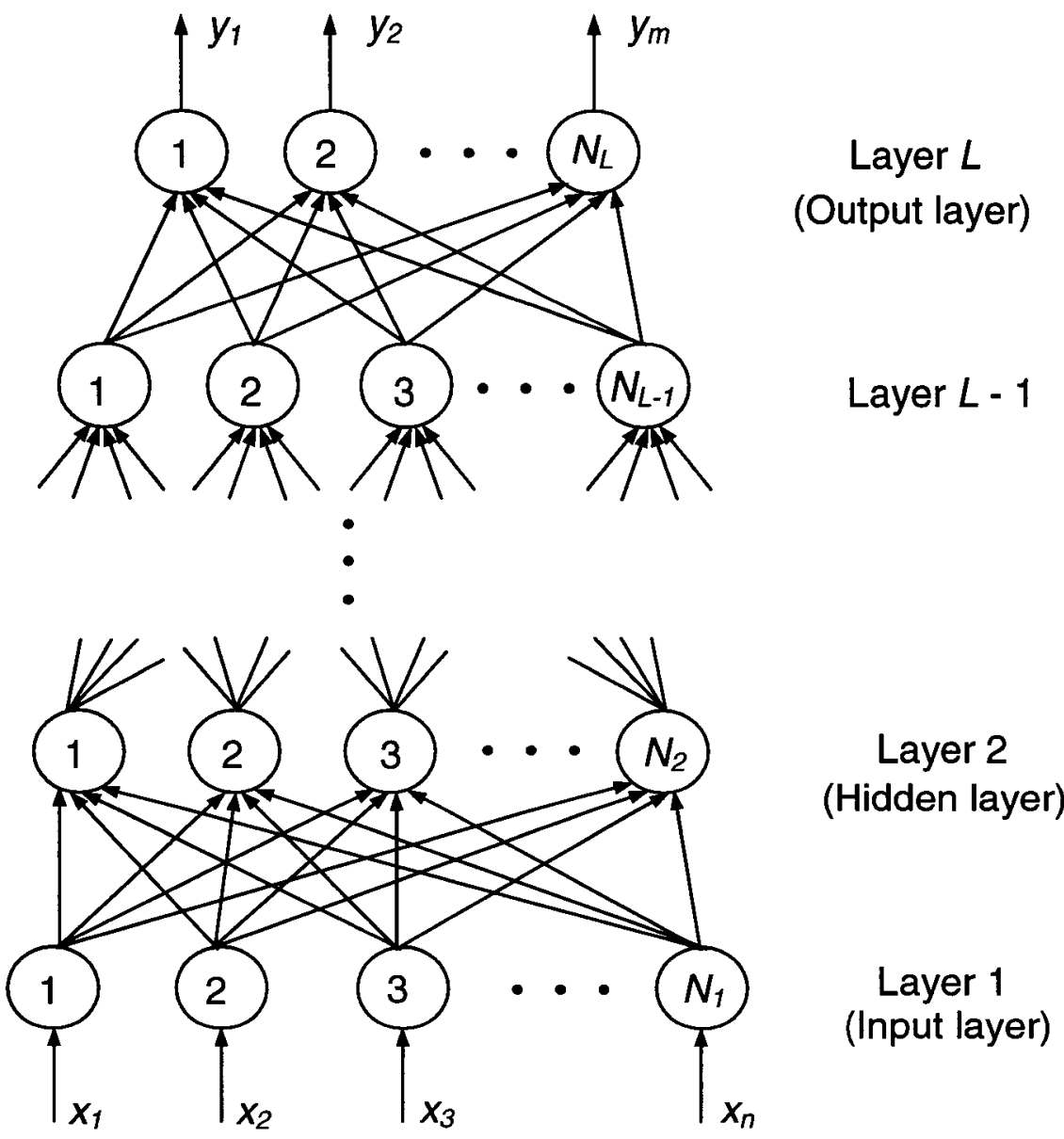

Figure 2.1 Multilayer Perceptrons (MLP) Neural Network Structure. Typically, a MLP network consists of an input layer, one or more hidden layers, and an output layer. 

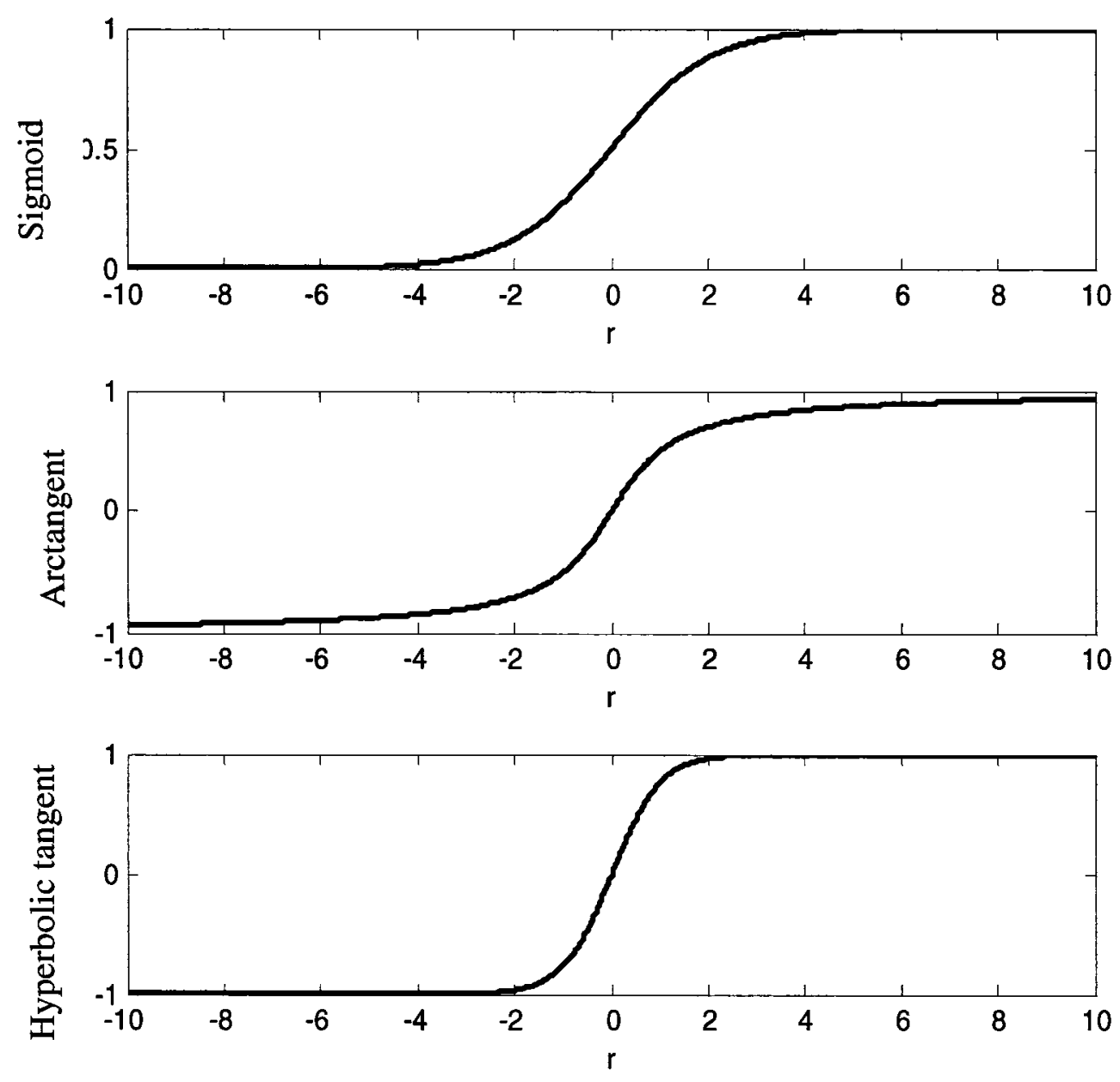

Figure 2.2 Commonly used nonlinear activation functions of hidden neurons in MLP network 
where $y_{k}$ represent the $k^{\text {th }}$ output of the MLP. The feedforword computation of MLP network, as shown in Eqs. (2.2), (2.3), and (2.7) is repetitively used during the training process of the MLP neural network as well as the final usage of the MLP model in microwave simulation and design. Through these simple computations that only consist of additions and multiplications, the MLP models can run much faster than the detailed EM/physics models of the microwave component.

One of the main reasons that make MLP a popular choice of microwave modeling is the universal approximation theorem of MLP proven by Cybenko [34] and Hornik et al. [35] in 1989. Essentially, according to the universal approximation theorem, provided sufficient hidden neurons, a 3-layer perceptron network is theoretically capable of approximating an arbitrary continuous, multi-dimensional real static nonlinear function to any desired accuracy. This theorem is the theoretical basis of the ANN modeling for microwave applications; however, the universal approximation theorem does not specify the number of hidden neurons required for a given problem. In theory, the number of hidden neurons depends on the degree of nonlinearities and the dimensions of the original problem. More nonlinear and high dimensional problems typically need more hidden neurons to get good model accuracy. But too many hidden neurons will result in too much freedom of the neural network weights that may cause the problem of over-learning [33]. Several algorithms have been introduced to find a proper size of MLP network such as constructive algorithm and network pruning [36]. Recently, the automatic model generation (AMG) algorithm was introduced to adaptively adjust the number of hidden neurons based on the observations of over-learning and under-learning 
during the automated model development [37].

In practical RF/Microwave applications, MLP networks with one or two hidden layers are commonly used. The choice between 3-layer and 4-layer MLP network depends on the patterns of the problem and model generalization capabilities. Intuitively, 4-layer perceptron network, i.e. the network with two hidden layers, is a better choice for tasks that have certain common localized behavior in different regions of the problem space. For the same task, a 3-layer perceptron network may need lots of hidden neurons to repeat the same behavior in different input regions. In [38], it is pointed out that 3-layer perceptron networks are preferred for function approximations where the generalization capability is a major concern.

\subsection{Knowledge-Based Nonlinear Dynamic}

\section{Modeling}

The knowledge-based methods have been mainly formulated to address the passive component modeling [33]. There are four existing knowledge-based methods introduced in this section. They are the milestones in the area of neural based circuit modeling. As we know, compared to detailed physical/EM models [39] - [41], equivalent circuit models are much faster but are less flexible, and their uses are often limited to the type of devices for which the model was developed. In order to develop a model, one has to firstly create a model structure and then do parameter extraction to determine parameters in the model. While parameter extractions can be done by computer-based optimizations, model structure creation is often mainly human based. The conventional process is to use 
human experience and skill to create an equivalent circuit topology and to create a nonlinear function for each of the nonlinear branches in the equivalent circuit. This process is trial-and-error based and is intensively rely on human calculation. To reduce the human laboring, several methods of nonlinear modeling by neural networks have been proposed [42]. Neural models are used to represent the DC characteristics of a physics-based MESFET [43], and small-signal behavior of an HBT [27]. It has been applied to large-signal transistor modeling [9] [10] and global modeling [44]. A recurrent neural network method using discrete time domain formulation was proposed in [27] to model nonlinear circuits and devices. These methods represent important steps towards automating the device modeling process. However because the neural networks learn the device behavior without using existing device formula, more training data is needed, or the reliability of the model will be poor. It would be more desirable to utilize the existing models and to use neural networks to complement what is missing in the existing models.

\subsubsection{Knowledge Based Neural Networks}

Microwave modeling using MLP neural networks is often referred as black box modeling due to the fact that MLP structure contains no microwave-dependant knowledge information of the original microwave device/circuit. Though the black box modeling is easy to implement, it usually requires a large amount of training data, which is often very expensive to generate for microwave applications [33]. The microwave knowledge complements the capability of learning and generalization of neural networks by providing additional information, which may not be adequately represented in a limited set of training data. Such knowledge 
becomes even more valuable when the neural model is used to extrapolate beyond training data region. By incorporating microwave empirical or semi-analytical information into the internal structure of neural networks, a Knowledge Based Neural Network (KBNN) was presented for RF/Microwave modeling and design for enhancing the neural model accuracy and reducing the need for massive training data [43].

In KBNN structure, as illustrated in Fig 2.3, the microwave knowledge in the form of empirical formulas is inserted as a part of the overall neural network structure. There are 6 layers in the structure that are not fully connected to each other. These 6 layers are denoted as input layer $\boldsymbol{X}$, knowledge layer $\boldsymbol{K}$, boundary layer $\boldsymbol{B}$, region layer $\boldsymbol{R}$, normalized layer $\overline{\boldsymbol{R}}$, and output layer $\boldsymbol{Y}$. The input layer $\boldsymbol{X}$ accepts parameters $\boldsymbol{x}$ from outside of the model. The knowledge layer $\boldsymbol{K}$ is the place where existing knowledge resides in the form of single or multidimensional functions $\psi$. Output of knowledge neuron $i$ in the $\boldsymbol{K}$ layer is given by [43]

$$
k_{i}=\psi_{i}\left(\boldsymbol{x}, \boldsymbol{w}_{i}\right), \quad i=1, \ldots, N_{k}
$$

where $\boldsymbol{x}$ is the vector including neural network inputs $x_{i}\left(i=1, \ldots, N_{x}\right), \quad N_{k}$ is the number of knowledge neurons, and $w_{i}$ is the vector of all the parameters in the knowledge formula. The knowledge function $\psi_{i}\left(\boldsymbol{x}, \boldsymbol{w}_{i}\right)$ is usually in the form of empirical or semi-analytical functions. As an example, the mutual inductance of a transmission line is a function of conductor width, separation between conductors and height of substrate [43]. The boundary layer $\boldsymbol{B}$ can incorporate knowledge in the form of problem dependent boundary functions $B$; or in the absence of boundary 
knowledge just as linear boundaries. Output of the $i^{\text {th }}$ neuron in this layer $b_{i}$ is described by

$$
b_{i}=B_{i}\left(x, v_{i}\right), \quad i=1, \ldots, N_{b}
$$

where $\boldsymbol{v}_{i}$ is a vector of all parameters in $B_{i}$ representing an open or closed boundary in the input space $\boldsymbol{x}$, and $N_{b}$ is the number of boundary neurons [43]. The region layer $\boldsymbol{R}$ contains neurons to construct regions from boundary neurons, whose outputs $r$ can be computed as

$$
r_{i}=\prod_{j=1}^{N_{b}} \sigma\left(\alpha_{i j} b_{j}+\theta_{i j}\right), \quad i=1, \ldots, N_{r}
$$

where $\alpha_{i j}$ and $\theta_{i j}$ are the scaling and bias parameters, respectively, and $N_{r}$ is the number of region neurons. Here $\sigma$ denotes a sigmoid function [33]. The normalized region layer $\overline{\boldsymbol{R}}$ contains rational function based neurons [74] to normalize the outputs of region layer,

$$
\bar{r}_{i}=\frac{r_{i}}{\sum_{j=1}^{N_{r}} r_{j}}, \quad i=1, \ldots, N_{\bar{r}}, \quad \text { where } N_{\bar{r}}=N_{r}
$$

The output layer $\boldsymbol{Y}$ contains second order neurons combining the outputs from both knowledge neurons and normalized region neurons [40]

$$
y_{i}=\sum_{i=1}^{N_{k}} \beta_{j i} k_{i}\left(\sum_{s=1}^{N_{\bar{T}}} \rho_{j i s} \bar{r}_{s}\right)+\beta_{j 0}, j=1, \ldots, N_{y}
$$


where $\beta_{j i}$ reflects the contribution of the $i^{\text {th }}$ knowledge neuron to output neuron $y_{j}$ and $\beta_{j 0}$ is the bias parameter. $\rho_{j i s}$ is a trainable region selector. If it is 1 it means that region $\bar{r}_{s}$ is the effective region of the $i^{\text {th }}$ knowledge neuron contributing to the $j^{t h}$ output. A total of $N_{\bar{r}}$ regions are shared by all the output neurons. As a special case, if we assume that each normalized region neuron selects a unique knowledge neuron for each output $j$, the function for output neurons can be simplified as

$$
y_{j}=\sum_{i=1}^{N_{k}} \beta_{j i} k_{i} \bar{r}_{i}+\beta_{j 0}, j=1, \ldots, N_{y}
$$

Training parameters $\psi$ for the entire KBNN model includes [43]

$$
\begin{aligned}
\psi= & {\left[\boldsymbol{w}_{i} i=1, \ldots, N_{k} ; \boldsymbol{v}_{i} i=1, \ldots, N_{b} ; \alpha_{i j}, \theta_{i j} i=1, \ldots, N_{r}, j=1, \ldots, N_{b} ;\right.} \\
& \left.\beta_{i j} j=1, \ldots, N_{y}, i=0, \ldots, N_{k} ; \rho_{j i k} j=1, \ldots, N_{y}, i=1, \ldots, N_{k}, k=1, \ldots, N_{\bar{r}}\right]
\end{aligned}
$$

The prior knowledge embedded in $\mathrm{KBNN}$ gives neural network additional information about the original microwave problem. Very often such information may not be well represented in the existing training data. As such, KBNN models have better reliability when only limited number of the training data is available. When used outside the model training range, the KBNN models also have better extrapolation capabilities as compared to the conventional black box neural models. The KBNN technique has been applied to model both passive and active microwave components with improved accuracy, reduced cost of model development and less need of training data over conventional neural models for microwave design [40]. 


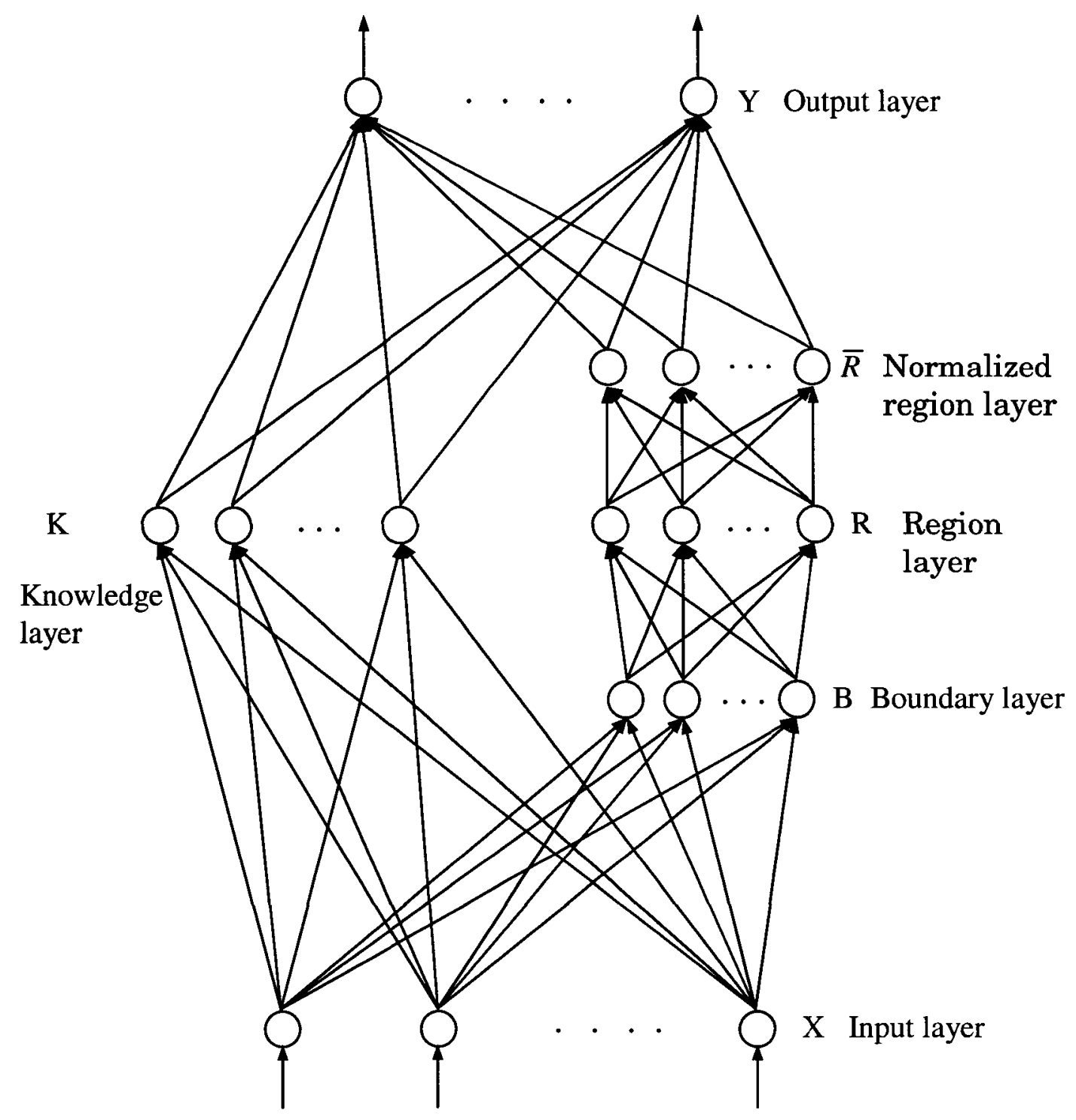

Figure 2.3 Knowledge Based Neural Network (KBNN) structure. Typically a KBNN consists of six layers. 


\subsubsection{Space Mapping (SM)}

Space mapping [29] is an advanced optimization concept and space mapping has successfully achieved substantial computation speedup in otherwise expensive optimizations of microwave components and circuits [9] [10]. Modeling techniques combining space mapping and neural networks have also been developed, demonstrating great efficiency in both passive modeling (small-signal device modeling) [29] and active modeling (large-signal device modeling) [6] problems.

SM combines the computational efficiency from the coarse models and the accuracy from the fine models. Usually the coarse models are typically empirical or equivalent circuit engineering models. They are fast and easy to model but often have a limited validity range for their parameters. Beyond the validity range, the results of coarse model simulation will become inaccurate. At the same time, physics/EM simulator or measurement can provide detailed or fine models, which are accurate but time consuming.

Space mapping technique establishes a mathematical link between the coarse and the fine models, and directs the bulk of CPU intensive evaluations to the coarse model, while preserving the accuracy and confidence offered by the fine model.

Let the vectors $x_{c}$ and $x_{f}$ represent the design parameters of the coarse and fine models, respectively, and $R_{c}\left(x_{c}\right)$ and $R_{f}\left(x_{f}\right)$ be the corresponding model responses. $R_{c}$ is much faster to calculate, but less accurate than $R_{f}$. The aim of SM optimization is to find an approximate mapping $P$ from the fine model parameter space $x_{f}$ to the coarse model parameter space $x_{c}$, i.e., $x_{c}=P\left(x_{f}\right)$ such that $R_{c}\left(P\left(x_{f}\right)\right) \approx R_{f}\left(x_{f}\right)$. As illustrated in Fig. 2.4, the mapping function $P\left(x_{f}\right)$ is realized by a neural network 
$\boldsymbol{x}_{c}=f_{A N N}\left(x_{f}, w\right)$. The coarse model then produces the overall output $y=R_{f}\left(x_{f}\right)$, which should match the training data.

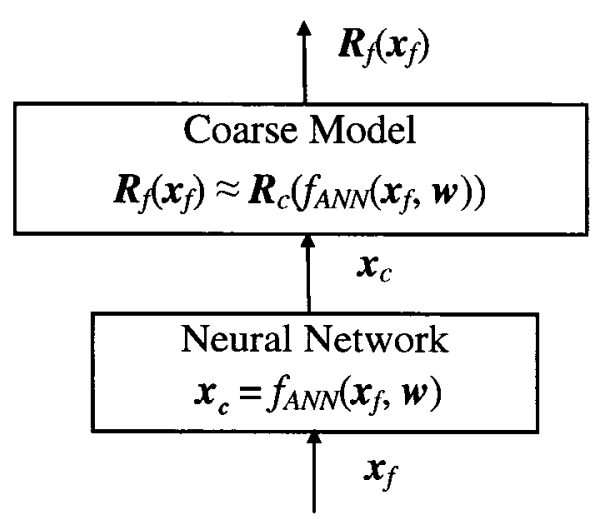

Figure 2.4 Structure of space mapping model.

\subsubsection{Difference Method (DM) for Nonlinear Modeling}

Generally in the DM method, empirical function part gives an approximation of the output and neural network will be trained to learn the difference between the accurate data and the approximate model. Let the 2 port functional relationship $\left[i_{\text {gequ }}, i_{\text {dequ }}\right]=$ $f_{\text {equ }}\left(v_{g}, v_{d}\right)$ represent an existing equivalent circuit model, where $v_{g}$ and $v_{d}$ are the terminal voltages, and $i_{g}$ and $i_{d}$ represent the terminal currents of the device. In typical FET modeling, $v_{g}$ and $v_{d}$ represent gate and drain voltages and $i_{g}$ and $i_{d}$ represent gate and drain currents. The relationship between these terminal voltages and currents are dynamic nonlinear relationships. The modeling structure combining existing models and neural network is proposed as shown in Fig. 2.5. 


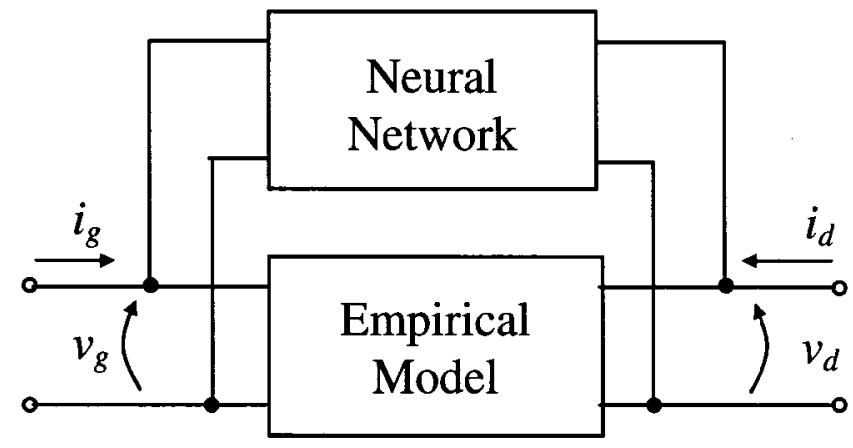

Figure 2.5 Knowledge-based structures for nonlinear modeling utilizing DM concept.

Since the neural network model should also be nonlinear dynamic model, direct use of MLP or any of the methods is not suitable. The method of constructing the neural network part is based on a recent dynamic neural network (DNN) [45] method and this model is the complete nonlinear large signal model that can be used for time- and frequency-domain purposes. The DNN can be developed without assuming any specific current-charge format, and has the potential to represent higher order dynamics. The format of the model is:

$$
\begin{aligned}
& \dot{v}_{1}(t)=v_{2}(t) \\
& \quad \vdots \\
& \dot{v}_{n-l}(t)=v_{n}(t) \\
& \dot{v}_{n}(t)=f_{A N N}\left(v_{n}(t), v_{n-I}(t), \cdots, v_{l}(t), u^{(n)}(t), u^{(n-1)}(t), \cdots, u(t)\right)
\end{aligned}
$$


where the inputs and outputs of the model is $\boldsymbol{u}(t)$ and $\boldsymbol{y}(t)=\boldsymbol{v}_{1}(t)$, respectively. And a circuit representation of the model is shown in Fig. 2.6.

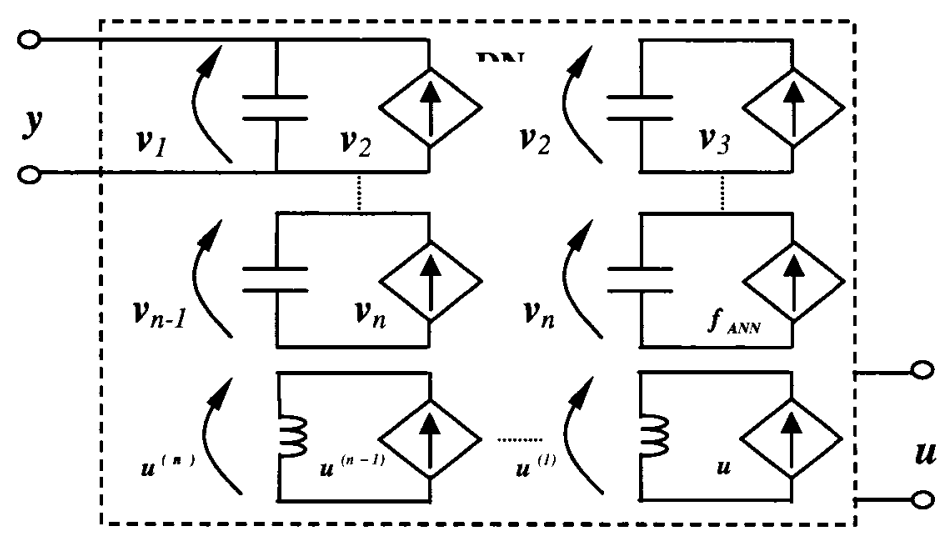

Figure 2.6 Circuit representation of the DNN model.

The model development includes 3 phases, data preprocessing of DC and bias dependent S-parameter data, neural network training, and the reconstruction of the overall model for the use in circuit design.

Phase 1: Compute the difference between the device data and those from the existing equivalent circuit model. Let the DC result data $\left(I_{g}\right.$ and $\left.I_{d}\right)$ be represented by $\Delta I_{g}$ and $\Delta I_{d}$, respectively. In order to train the $\mathrm{AC}$ behavior, we also use the S-parameter data at various bias points for training. To be able to do the direct computation, we convert the S-parameter into Y-parameter for both the device and model data. We then compute the difference between the Y-parameter of the device and that of the existing model. Let $\Delta R Y_{11}, \Delta I Y_{11}, \Delta R Y_{12}, \Delta I Y_{12}, \Delta R Y_{21}, \Delta I Y_{21}, \Delta R Y_{22}, \Delta I Y_{22}$ represent the result of such 
difference. Now we have $V_{g}, V_{d}$, frequency, $\Delta I_{g}, \Delta I_{d}, \Delta R Y_{11}, \Delta I Y_{11}, \Delta R Y_{12}, \Delta I Y_{12}, \Delta R Y_{21}$, $\Delta I Y_{21}, \Delta R Y_{22}$ and $\Delta I Y_{22}$ for each bias point. This can be used as training data for Phase 2.

Phase 2: Train the neural model to learn the difference data processed in Phase 1 . The objective of training is to minimize the least square difference of $\left[\Delta I_{g}, \Delta I_{d}, \Delta R Y_{11}, \Delta I Y_{11}\right.$, $\Delta R Y_{12}, \Delta I Y_{12}, \Delta R Y_{21}, \Delta I Y_{21}, \Delta R Y_{22,} \Delta I Y_{22}$ ] between the current-charge neural model (or DNN model) and that of the data from Phase 1. For the DNN based neural model, we use a combined time and frequency domain training technique [45] to train the DNN to learn the difference data.

Phase 3: Construct the overall model by connecting the equivalent circuit and the DNN models in parallel. When $v_{g}$ and $v_{d}$ signals are supplied to the model by user, the equivalent circuit model part will first produce a solution of $i_{g e q u}$ and $i_{\text {dequ }}$. In the DNN based approach, the DNN will produce a higher order correction of $\Delta i_{g}$ and $\Delta i_{d}$. With parallel connection, the total current for gate and drain terminals from the model will be $i_{g}=i_{g e q u}+\Delta i_{g}$ and $i_{d}=i_{d e q u}+\Delta i_{g}$, respectively. If the training in Phase 2 was done accurately, then this solution will be an accurate representation of the device behavior. This is achieved even though that the original equivalent circuit is not accurate.

\subsubsection{Neual-Space Mapping (Neural-SM)}

Artificial Neural Networks and Space Mapping [33] [29] are two recent developments in the microwave $\mathrm{CAD}$ area to address the growing challenges in today's microwave modeling, simulation and optimization. Neural networks can be trained to learn from microwave data, and the trained neural network can be used as microwave model providing fast solutions to the task it learned [33]. It has been applied to a variety of microwave modeling problems including both passive [29] 
and active [6] modeling. Because neural network computation is fast and it can generalize from data, it allows the model development even when component formulas are unavailable. Space mapping, on the other hand, is an attractive concept for circuit design and optimization, which is combining the computational efficiency of coarse models with the accuracy of fine models. The coarse models are typically empirical equivalent circuit engineering models, which are fast but have limited validity range for their parameters, beyond which the simulation results may become inaccurate. Meanwhile, detailed fine models can be provided by EM simulator or direct measurement. Space mapping establishes a mathematical link between coarse and fine models to achieve an optimization of fine accuracy without extensive use of fine model.

Recently, space mapped neuromodeling technique was proposed combining neural networks with space mapping [29] [46]. A neural network is trained to map the coarse model towards fine model data. The result is a model with accuracy near fine model and the speed of the coarse model. The technique has been applied to passive component modeling such as bends, high temperature superconductor filters [12] [46] and embedded passives in multilayer printed circuits [47]. Overall in the Neural-SM method, the neural network module maps the original problem input space into a coarse (empirical) model input space. The coarse model then produces the overall output with improved accuracy.

In this section, the neuro-space mapping technique for automatic modeling of large-signal nonlinear devices is presented. Let us firstly define a given existing device model as coarse model. The proposed technique then will automatically 
adjust and modify the coarse device model such that after space mapping, the mapped model will match the fine device data.

To achieve the large signal device modeling, the neural network mapping is formulated using voltage and current signals in the model. After the efficient training of the neuro-space mapping with DC and bias dependent S-parameter data, the trained model is then used in large-signal harmonic balance simulation.

Suppose that the existing or available equivalent circuit model gives out the rough approximation of our device, and let us call it the coarse model. The fine model in this case is only a fictitious model implied by actual device data from measurement or detailed/expensive device simulator. Let $\boldsymbol{v}_{c}=\left[v_{c 1}, v_{c 2}\right]^{\mathrm{T}}$ and $\boldsymbol{i}_{c}=\left[i_{c l}, i_{c 2}\right]^{\mathrm{T}}$ represent the terminal currents and voltage signals of the coarse device model, respectively. Let the terminal currents and voltages of the fine model be defined as $v_{f}=\left[v_{f 1}, v_{f 2}\right]^{\mathrm{T}}$, and $i_{f}=\left[i_{f 1}\right.$, $\left.i_{f 2}\right]^{\mathrm{T}}$, respectively. For simplicity, $v_{c}$ and $\boldsymbol{i}_{c}$ are called coarse signals and $v_{f}$ and $\boldsymbol{i}_{f}$ are called fine signals.

A 2-port formulation for a nonlinear Neuro-SM structure is shown in Fig. 2.7. The voltage signals for the overall model, i.e., $v_{f 1}$ and $v_{f 2}$ are mapped into the voltages in the coarse model such that the modified coarse model response $\boldsymbol{i}_{c}$ will match the fine signal $i_{f}$. A neural network has been exploited to represent the unknown mapping function in the Neuro-SM model:

$$
v_{c}=f_{A N N}\left(v_{f}, w\right)
$$

where $f_{A N N}$ represents a feedforward the neural network and $\boldsymbol{w}$ is a vector containing all internal weights of the neural network. In the neuro-space mapping formulation, the 
neural network function $f_{A N N}$ is implemented as voltage controlled voltage sources as shown in Fig. 2.7. Unit current controlled current sources are used to pass the coarse current $\boldsymbol{i}$ to the fine current $\boldsymbol{i}_{f}$, in order to make the neuro-space mapping model consistent with Kirchhoff's Laws for circuit simulation.

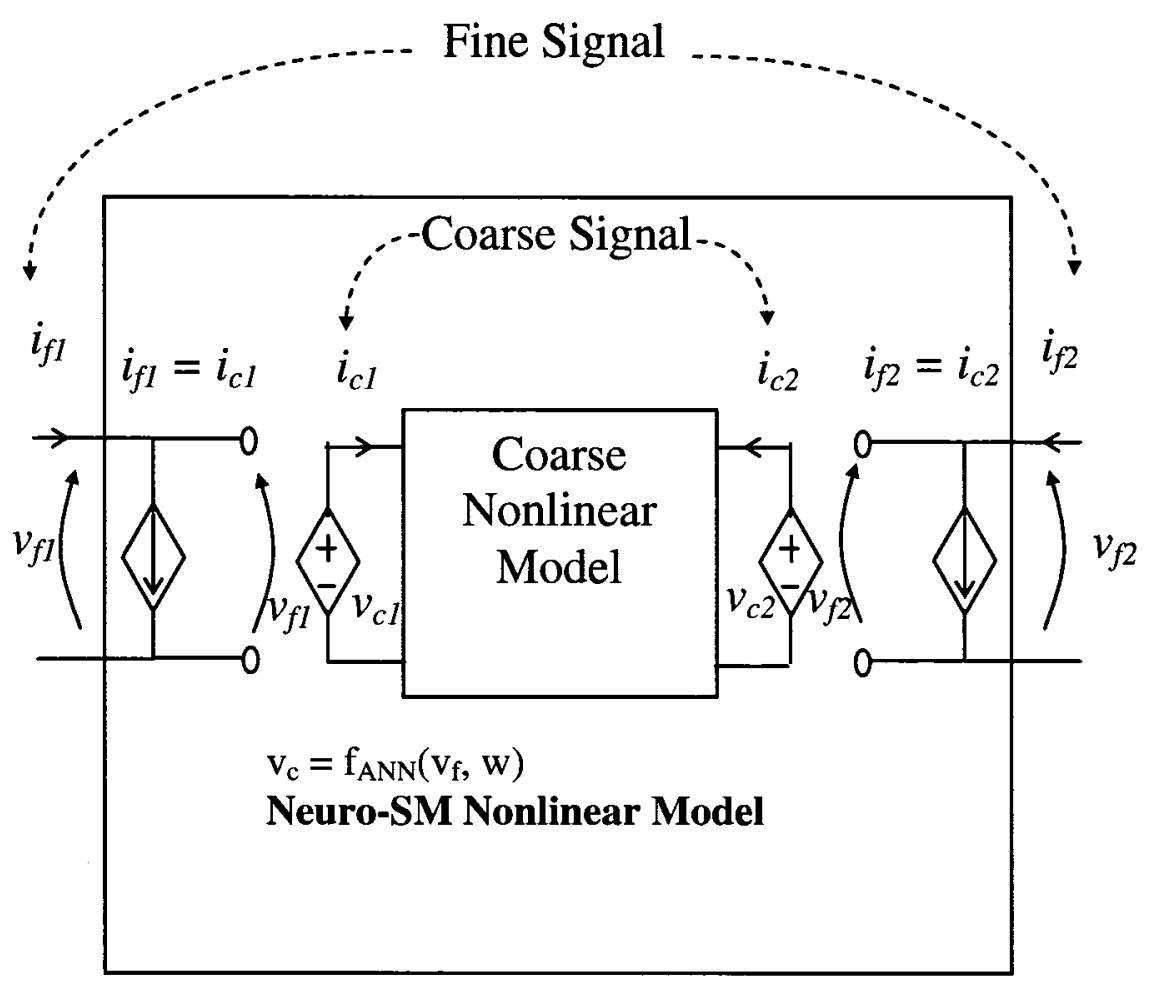

Figure 2.7 General 2-port Neuro-SM nonlinear model. 
The mapping for DC voltages $V_{c, D C}$ and $V_{f, D C}$ is directly achieved by the neural network as:

$$
\boldsymbol{V}_{c, D C}=f_{A N N}\left(V_{f, D C}, w\right)
$$

The small signal S-parameters are mapped via the mapping relationship of the $\mathrm{Y}$ matrices between the coarse model $\boldsymbol{Y}_{c}$ and fine model $\boldsymbol{Y}_{f}$, as

$$
\boldsymbol{Y}_{f}=\boldsymbol{Y}_{c} \cdot\left(\left.\frac{\partial \boldsymbol{f}_{A N N}^{T}\left(v_{f}, \boldsymbol{w}\right)}{\partial \boldsymbol{v}_{f}}\right|_{v_{f}=V_{f S B i s}}\right)^{T}
$$

where the derivative of $f_{A N N}$ is obtained at bias point $V_{f, B i a s}$ using the adjoint neural network. As for large-signal case, the mapping of harmonic signals between the coarse model $V_{c}(k \omega)$ and fine model $V_{f}(l \omega)$ is:

$$
\begin{gathered}
\boldsymbol{V}_{c}(k \omega)=\frac{1}{N_{T}} \sum_{n=0}^{N_{T}-1} f_{A N N}\left(\sum_{l=0}^{N_{H}} V_{f}(l \omega) \cdot W_{N}^{-(l \omega)(n T)}, \boldsymbol{w}\right) \cdot W_{N}^{(k \omega)(n T)} \\
k=0,1, \ldots, N_{H} .
\end{gathered}
$$

where $\omega$ is fundamental frequency, $N_{H}$ is number of harmonics, $T$ is time interval and $N_{T}$ is the number of time points. $W_{N}$ is defined as $e^{-j 2 \pi}$. 
The goal of the Neuro-SM model training is to find the optimal weights $\boldsymbol{w}$ such that the overall model can best match the fine data and the overall training has two phases, initialization and formal training. In the initialization phase, initialization the neural network by a preliminary training to learn unit mapping is needed, i.e., $\boldsymbol{v}_{\boldsymbol{c}}$ $=v_{f}$. Training data for unit mapping is generated by sampling $\left[v_{c l}, v_{c 2}\right]$ in a grid form to cover the entire operation region of the original device. The initialization phase ensures that the overall Neuro-SM model has equal accuracy as the coarse model, before the formal training of the neural network is carried out. In the formal training phase, the neural network internal weights $w$ are adjusted such that the Neuro-SM model can match device data. In this way, Neuro-SM model is guaranteed to exceed the performance of the given coarse model.

The overall training error is calculated based on the total difference between all available device data (such as DC and bias-dependent S-parameters) and the NeuroSM model. The derivatives of the training error required during the neural network training is obtained by differentiating the mapping relationships of (2.17)-(2.19). In particular, the derivatives for $f_{A N N}$ are achieved by using the efficient adjoint neural network sensitivity technique [48].

After training, the Neuro-SM model can be used by user or circuit simulator. The neural network internal weights $\boldsymbol{w}$ are fixed. The voltage/current relationship of the model required by user or circuit simulator is that between $\boldsymbol{v}_{f}$ and $\boldsymbol{i}_{f}$, which is obtained from Neuro-SM model through the mapping of coarse model signals. 


\subsection{Brief Overview of Conventional Statistical}

\section{Modeling Techniques}

In industry, the complicated and time-consuming $\mathrm{RF}$ and microwave circuit design requires reliable statistical models that are used to accurately represent the electrical performance variations of microwave devices because of the process parameter dispersions. The conventional statistical modeling techniques often use optimization-based parameter extraction [23] to obtain equivalent circuit parameters with the statistical properties correctly reflecting the physical variation effect of the actual devices [1].

The equivalent circuit parameters containing the statistical information are directly extracted from the measurement data in a given population of devices. Each set of measured data for each device, is converted to corresponding equivalent circuit parameters through a parameter extraction procedure. The statistical properties of the equivalent circuit parameters are then examined and the estimates of the statistics are calculated in mean, standard deviation for each parameter, and correlation among parameters. Finally, statistical models based on some multivariate or heuristic techniques [49] capable of recreating those statistics can be developed.

Usually the extracted equivalent circuit parameters cannot be considered as independent random variables and therefore the implementation of a multivariate distribution is required. The equivalent parameters often are correlated to each other [4] [8], so accurately reproducing means, standard deviations, and correlations 
among the responses of the equivalent circuit model is very important. However, the equivalent circuit parameters extracted from measurement data may not be unique solutions, which will lead to inaccurate representation of the actual distribution, even if the fit of the simulated responses to the corresponding measurements for individual device model is excellent. One solution to this has been discussed in [2] by fitting the cumulative probability distributions (CPDs) of the model responses to those of the measured data. Using the least and sufficient number of equivalent circuit parameters is the goal of the optimization, but this may not be easy to be achieved. Recently, artificial neural networks (ANNs) have been used as accurate and efficient statistical modeling methods [7] to overcome the uncertainty caused by optimization-based extraction.

Most of the statistical modeling approaches described above is based on DC and S-parameter measurements. With the increasing need of accurate characterization of nonlinear device behaviors, modeling techniques directly using large-signal measurement data have gained attentions. Reliable nonlinear statistical models are essential in circuit applications where bias point variations of active devices have a strong impact on overall yield; however, direct large-signal statistical modeling remains prohibitive because complete large-signal measurement for many devices is too expensive and time consuming. 


\subsection{Conclusions}

The existing neural network based modeling techniques for microwave/RF circuit design that are relevant to this thesis work, have been reviewed. Neural network based models can be used to achieve a significant speedup of microwave/RF simulation and optimization, by replacing electronic and microwave component models, which are represented by detailed EM equations. These neural models can be trained with the corresponding EM data; however, most of the existing neural network structures are of black box type without any physicaldependent information embedded, and need a large amount of training data to get an accurate model, which results in high cost model development. Knowledge-based neural network is one solution to such problem. Particularly, Neural-SM shows the excellence and potentials for large-signal modeling thus we developed the proposed technique for large-signal statistical modeling based on the concept of Neural-SM. 


\section{Chapter 3}

\section{Proposed Linear Dynamic Space}

\section{Mapping Technique}

The proposed linear dynamic space mapping technique uses the Neuro-Space Mapping [6][29] concept. Instead of combining a neural network structure to the nominal model, a simpler set of linear dynamic functions is inserted. In this way, it aims to model the accurate statistical large-signal with only one set of large-signal measurement and combining with many supplementary sets of DC and biasdependent S-parameter measurements.

Now the proposed statistical model is clearly composed of a coarse model, which is the large-signal nominal model, and a fine model, which is the linear dynamic space mapping. As we pointed out before, the large-signal nominal model is developed using one complete set of large-signal measurement data to represent the nominal performance of a given device population while the linear dynamic space mapping is formulated to take into account of the statistical properties. The coefficients of the linear dynamic mapping functions are the statistical parameters extracted by matching the DC and bias-dependent S-parameter data of the given 
device population. In other words, the coarse model is used to stand for the largesignal nonlinear behavior and at the same time the statistical variations around the coarse model are clarified by the linear dynamic mapping functions.

One assumption for the proposed linear dynamic space mapping technique is that the variations of the equivalent circuit parameters for the given device population should be a small percentage around their nominal values, then a meaningful simple set of linear dynamic space mapping functions can be extracted from the DC and small-signal data to approximate the large-signal statistical variations. Otherwise, if the above assumption is violated, a simple linear mapping will not be able to precisely represent the large-signal variations. Thus, a complicated and tedious linear mapping with much higher dynamic order is required. Usually when the order goes high, the optimization will take much longer CPU time and the coefficients of the statistical mapping from the extraction will be much harder to analysis or regenerate.

\subsection{Linear Dynamic Space Mapping Technique}

The following starts from the problem statement. Let's consider a device population and we can have $N$ sets of inputs and outputs information. Because of the manufacture process, we are also going to have $N$ sets of statistical varied physical or geometrical parameters around some mean values, such as the gate length, width, 
and oxide permittivity, etc. for a transistor. The illustration of the statistical modeling problem is shown in Fig 3.1.

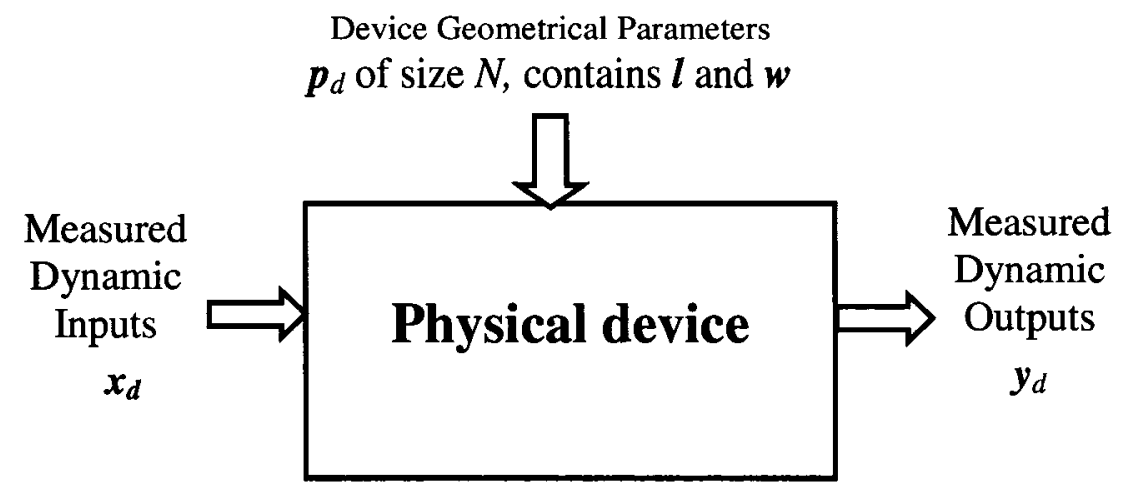

(a)

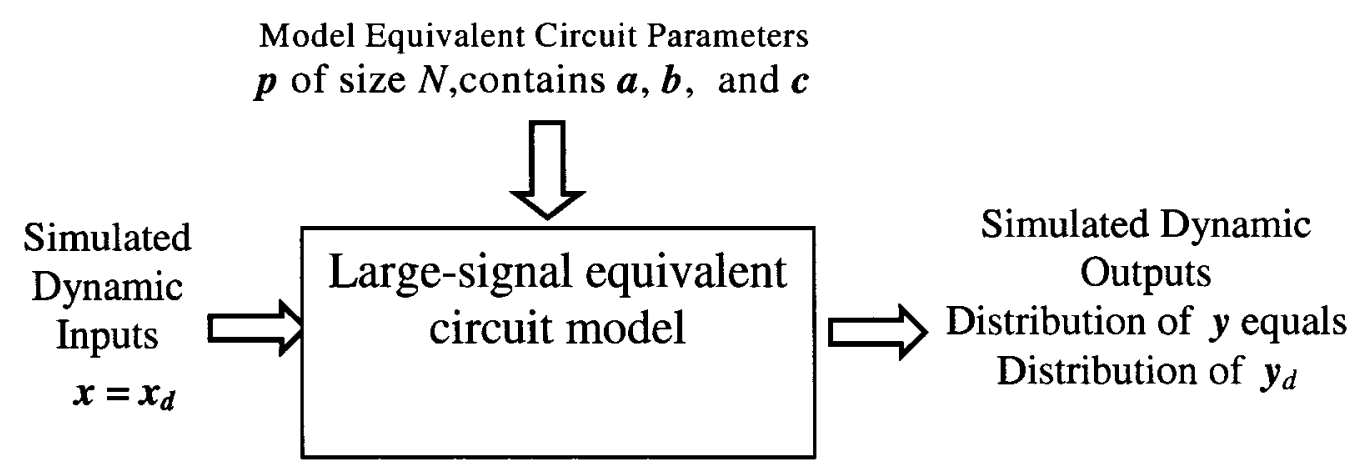

(b)

Figure 3.1 General large-signal statistical modeling. (a) Device population.

(b) Simulated model population. 
Let's use vector $\boldsymbol{x}_{d}$ to represent the actual dynamic inputs to the device population and a vector $\boldsymbol{y}_{d}$ represent the dynamic outputs corresponding to the input $\boldsymbol{x}_{d}$. Also let vector $\boldsymbol{p}_{d}$ be the physical or geometrical parameters that statistically vary among devices due to the foundry process. Assume in a general 2-port nonlinear device case of population size $N, \boldsymbol{x}_{d}$ represents the dynamic input to the device contains voltage inputs from port one and port two, i.e. $\boldsymbol{V}_{1}$ and $\boldsymbol{V}_{2}$. Use $\boldsymbol{y}_{d}$ to represent the dynamic output current signals, $\boldsymbol{I}_{2}$, from the port two. At the same time the physical or geometrical parameters $\boldsymbol{p}_{d}$ are said to be device length $\boldsymbol{l}$ and device width $\boldsymbol{w}$. To be able to model this set of device population, we need the same population size of models to match each single device in the general 2-port device population. Let's also define vector $\boldsymbol{x}$ and $\boldsymbol{y}$ as the dynamic inputs and outputs of the statistical models. Let $\boldsymbol{x}$ contain input voltage $v_{1}$ and $v_{2}$ while $y$ contain the output current $\boldsymbol{i}_{2}$. The equivalent circuit parameter $\boldsymbol{p}$ in the statistical model, used to represent the effect of the physical or geometrical variations, is to be an arbitrary function coefficients or equivalent circuit component values, $\boldsymbol{a}, \boldsymbol{b}$, and $\boldsymbol{c}$, with a multidimensional probability density function (PDF) $f_{p d f}(\boldsymbol{p})$ [50]. Now the statistical modeling problem is to find $\boldsymbol{p}$ and $f_{p d f}(\boldsymbol{p})$ based on the given measured data from the device population so if $\boldsymbol{x}=\boldsymbol{x}_{d}$, the outputs distribution of simulated model $\boldsymbol{y}$ will be able to accurately match the distribution of the measured outputs $\boldsymbol{y}_{\boldsymbol{d}}$ from the device population. Simply, if the outputs of the actual device have a Gaussian distribution [50] with certain mean and standard deviation values, the simulated statistical model should also perform the outputs to be the Gaussian distribution with the same mean and standard deviation values. 
As we described in Chapter 2, the conventional techniques for large-signal statistical modeling use the brute force parameter extraction as shown in Fig. 3.1(b). In this general 2-port nonlinear example, the existing large-signal modeling is summarized as the following steps:

Step1: Choosing a suitable large-signal equivalent circuit according to the characteristics of the device population.

Step2: $\quad$ The equivalent circuit parameters $\boldsymbol{a}, \boldsymbol{b}$, and $c$ as in the Fig $3.1 \mathrm{~b}$, may vary with different equivalent circuit models, and they are going to be considered as statistical parameters $p$, which will be used in the statistical modeling.

Step3: Given a device population of selected size $N$, the elements $\boldsymbol{a}, \boldsymbol{b}$, and $\boldsymbol{c}$ for $\boldsymbol{p}$ are extracted from $N$ different sets of DC, bias-dependent Sparameter, or large-signal measurement data correspond for each real device.

Step4: Once the extraction for all devices in the population gets finished, the means $(\mu)$, standard deviations $(\sigma)$, and correlation coefficients $(\rho)$ of $\boldsymbol{a}, \boldsymbol{b}$, and $\boldsymbol{c}$ for $\boldsymbol{p}$ are calculated, which represent the transformation of the statistical information of the population.

Step5: $\quad$ Based on the $\mu, \sigma$, and $\rho$, large-signal statistical model is constructed, and the distribution of the actual devices' performance is modeled.

However, this brute force modeling procedure is still not used in practice because 
the accuracy of the statistical modeling is directly based on the number of the device population or samples [50]. In general, we all know that the more the number of the device population going to be modeled, the more accurate statistical model we can get. Unfortunately, large-signal modeling and analysis is very time consuming even for a single device.

Since the conventional statistical modeling in large-signal is prohibitive because of time consuming, the proposed statistical linear dynamic space mapping model practically minimizes the large-signal measurements and analysis down to one single device and small-signal measurement for other devices. In this way, we can dramatically shrink our modeling time.

The simple illustration still with the same general 2-port example is shown in Fig. 3.2. The nonlinear large-signal nominal model is developed to fully fit a whole set of large-signal measurement data. This nominal model is needed to represent the large-signal behavior of the entire device population. This nominal model contains no statistical parameters. With the assumption that the parameter variations of the given population is usually a small percentage around their nominal values, a simple set of linear dynamic mapping functions is used to approximate the statistical variations in the population. This is because the equivalent circuit parameters are extracted independently from each device and the values for each set of equivalent circuit parameters according to each device is not unique; hence the distribution of each extracted parameter may not always simply be Gaussian. In other words, if the variation of the physical or geometrical parameters is very small, their effect on the distribution of each equivalent circuit parameter might become small, we thus can 
simply treat the distribution to be Gaussian, i.e. the values for each equivalent circuit parameter randomly varies around a certain nominal value; however, if the physical or geometrical parameters is large, we cannot guarantee the variations of the equivalent circuit parameters according to the given device population is still a small percentage around the nominal values, the parameter distribution then will no longer be necessarily Gaussian.

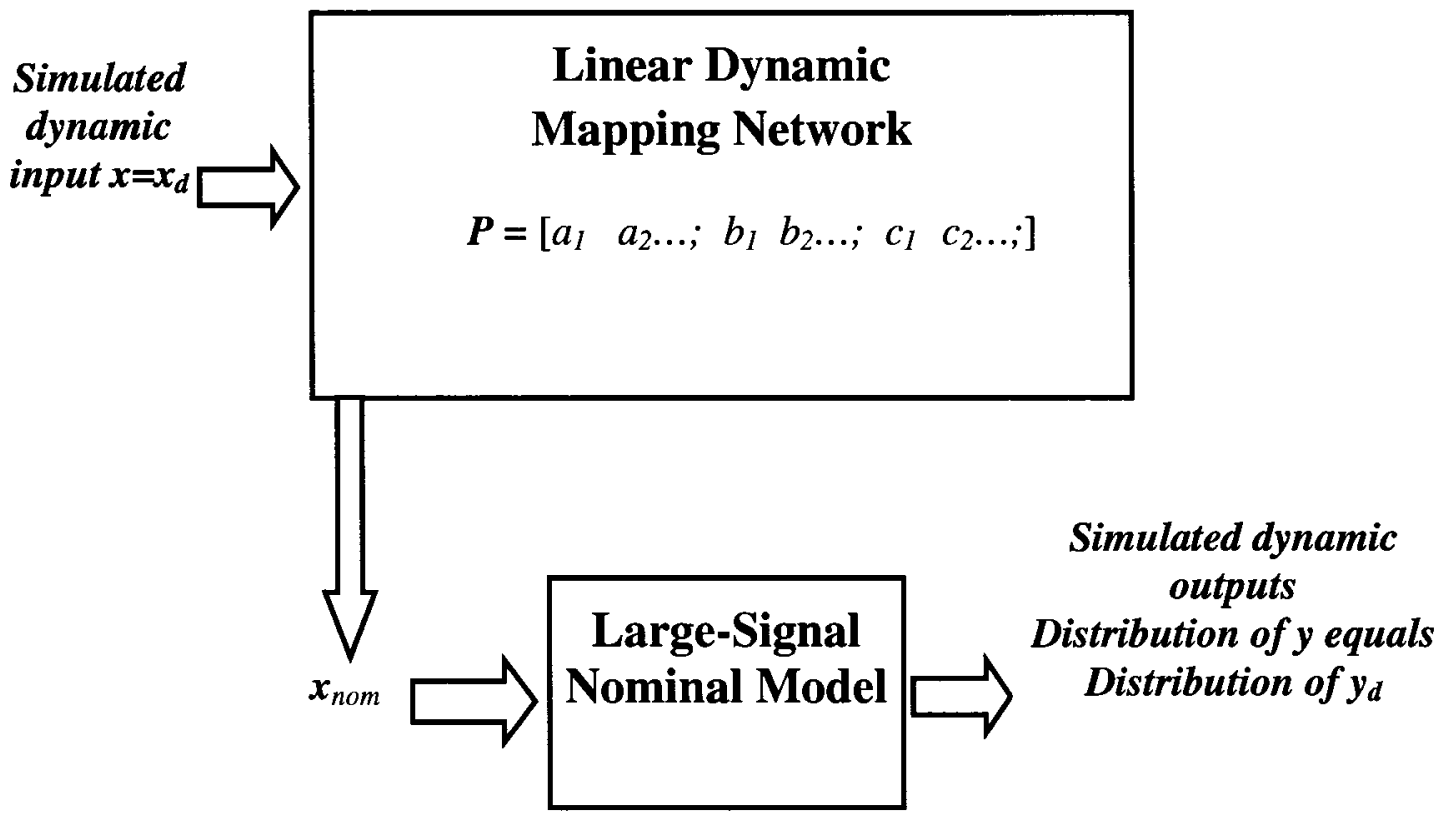

Figure 3.2 Large-signal statistical modeling approaches: statistical spacemapped model with linear dynamic mapping. 
The mapping coefficients $a, b$, and $c$ of the linear dynamic mapping functions are now considered as statistical parameters vector $\phi$, but no longer the equivalent circuit parameters $\boldsymbol{p}$. Similarly as in the conventional techniques, the statistical parameter $\phi$ is extracted from each device in the population from an optimization process based on the measured DC and bias-dependent S-parameter data. The $\mu, \sigma$, and $\rho$ of the statistical mapping parameters are then calculated from the extracted $\phi$ and the model can be used to approximate large-signal statistics. Because the model development is based only on one large-signal measurement (for nominal model) and mostly DC and biasdependent S-parameter measurements (for linear dynamic mapping function), it dramatically reduces the measurement cost while having the ability to reasonably represent large-signal statistical behavior; However, once again, this technique is developed based on the assumption of small variation of the physical or geometrical parameters, thus its accuracy may be limited if the assumption is violated where high dynamic orders are required leading to large number of $\phi$ and the distribution of the extracted mapping parameters may not be the desired Gaussian distribution.

\subsection{Detail in Proposed Linear Dynamic Space}

\section{Mapping Technique}

As we already pointed out, the nominal model is a nonlinear model developed from the large-signal measurement data. It can be an extracted equivalent circuit model [4] or a trained dynamic neural network (DNN) model [45]. This model 
contains no statistical parameters. It will be used as the coarse representation of the large-signal characteristics for the entire device population. In the meantime, the proposed technique uses the Neuro-space mapping concept in [6][7] by replacing the mapping neural network with a linear dynamic mapping to generalize the DC and S-parameter information for other devices.

The detail of the entire linear dynamic space-mapped technique is shown in Fig. 3.3 .

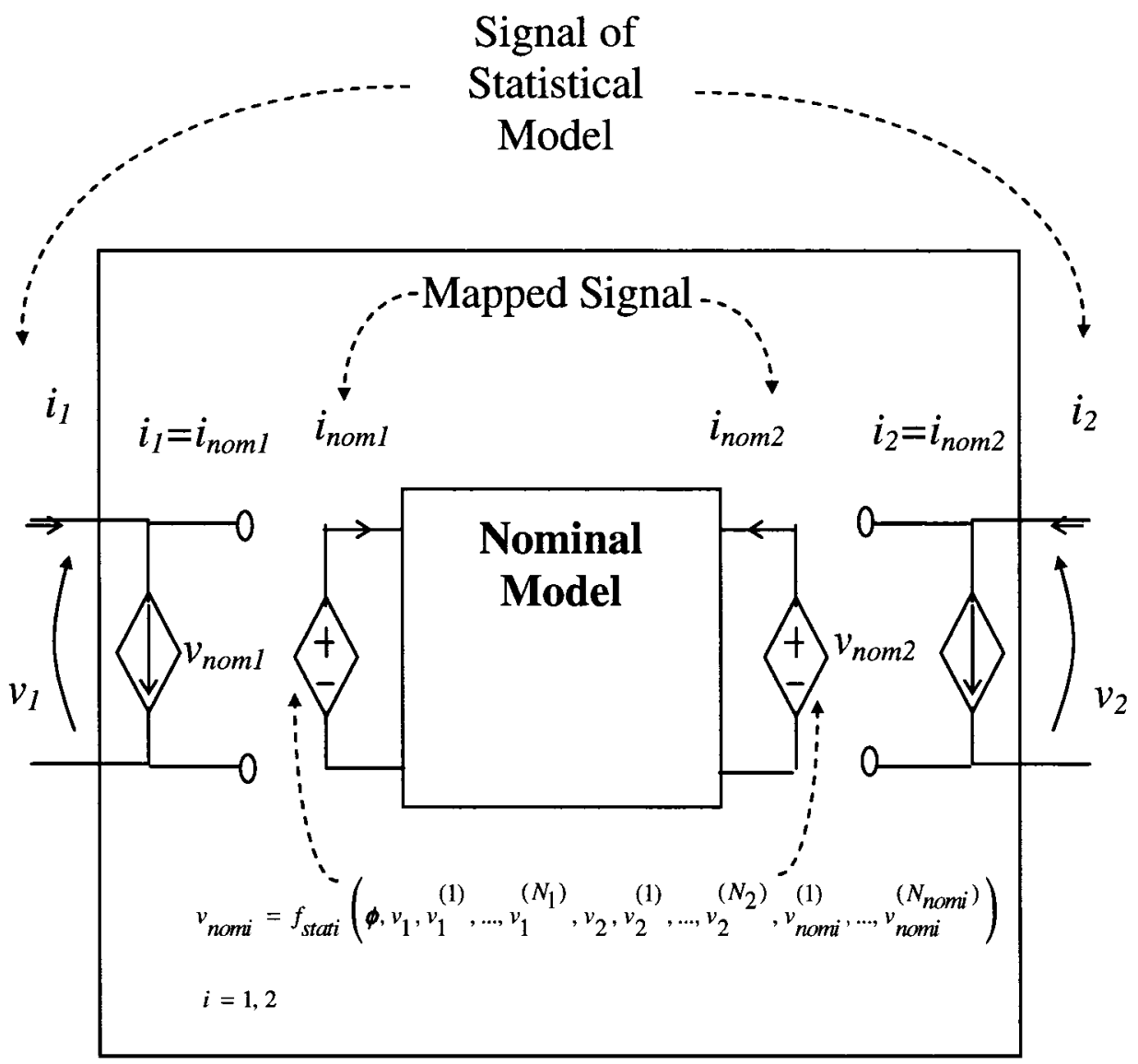

Figure 3.3 Two-port statistical space-mapped models. 
For the illustration purpose, a two-port device is examined. Let the terminal voltage and current signals (mapped signals) of the nominal model be defined as $\boldsymbol{v}_{\text {nom }}=\left[v_{\text {noml }}, v_{\text {nom } 2}\right]^{\mathrm{T}}$ and $\boldsymbol{i}_{\text {nom }}=\left[i_{\text {noml }}, i_{\text {nom } 2}\right]^{\mathrm{T}}$, respectively. Similarly define the terminal voltages and currents of the statistical model (original signals) as $v=\left[v_{l}\right.$, $\left.v_{2}\right]^{\mathrm{T}}$, and $\boldsymbol{i}=\left[i_{1}, i_{2}\right]^{\mathrm{T}}$, respectively. A linear dynamic mapping is implemented as the controlling functions of the voltage controlled voltage sources. Current controlled current sources are used to pass $\boldsymbol{i}_{n o m}$ to $\boldsymbol{i}$ in order to make the statistical model consistent with Kirchhoff's Laws, as seen from the external terminals of the overall model.

The mapping equation implemented in the controlled voltage sources is

$$
\begin{aligned}
v_{\text {nomi }} & =f_{\text {stati }}\left(\phi, v_{1}, v_{1}^{(1)}, \ldots, v_{1}^{\left(N_{1}\right)}, v_{2}, v_{2}^{(1)}, \ldots, v_{2}^{\left(N_{2}\right)}, v_{\text {nomi }}^{(1)}, \ldots, v_{\text {nomi }}^{\left(N_{\text {nomi }}\right)}\right) \\
= & \sum_{k=0}^{N_{1}} a_{i k} \cdot v_{1}^{(k)}+\sum_{k=0}^{N_{2}} b_{i k} \cdot v_{2}^{(k)}+\sum_{k=1}^{N_{\text {nomi }}} c_{i k} \cdot v_{\text {nomi }}^{(k)}+d_{i} \quad i=1,2
\end{aligned}
$$

where $v_{i}^{(k)}$ and $v_{n o m i}^{(k)}(i=1,2)$ are the $k^{\text {th }}$ derivatives of $v_{i}$ and $v_{n o n i}$ with respect to time $t$, respectively. $N_{i}$ and $N_{n o m i}$ are the derivative orders of the voltage signals at port $i(i=1,2)$ of the statistical model and the nominal model, respectively. $\phi$ is a vector of statistical parameters including $a_{i k}\left(k=1,2, \ldots, N_{1}\right), b_{i k}\left(k=1,2, \ldots, N_{2}\right), c_{i k}\left(k=1,2, \ldots, N_{\text {nomi }}\right)$, and $d_{i}$, where $i=1,2$ for all parameters.

For each device in the statistical population, DC and bias-dependent Sparameter data are measured. Parameter extractions of the $a^{\prime}$ s, $b$ 's, $c^{\prime}$ 's, and $d$ 's are 
performed based on the measurement data for each device. Once $\phi$ is extracted from all devices in the population, the means $(\mu)$, standard deviations $(\sigma)$, and the correlation coefficients $(\rho)$ of the statistical parameters $\phi$ will be calculated.

\subsection{Procedures of the Modeling}

To be able to make the proposed technique symmetrical practice and easily to follow, a logical and well-organized procedure is presented. It helps to understand how the modeling could be used in real industry process and makes the technique more clear.

Step 1. Select a nominal device which is a typical rough representative of a selected device population $N$ to be modeled. Perform large-signal measurement on the nominal device. Extract a large-signal nominal model from the large-signal measurement data.

Step 2. Perform load line analysis on the nominal model. Determine the operating frequency range of the nominal model. Select bias points along the load line and frequency points in the operating frequency range for DC and S-parameter measurements.

Step 3. Define the mapping function and derivative orders used in the mapping to minimize the optimization error of DC and small-signal. Combine the nominal model and the mapping as shown in Fig. 3.3. 
Step 4. For devices in the selected device population of size $N$, perform DC and S-parameter measurements at the selected bias and frequency points from Step 2. For each set of data, perform parameter extraction to obtain the mapping parameters $\phi$.

Step5. Perform DC and small-signal optimization error calculation. In other words, the distribution test between the model outputs and device outputs if not accurate enough, go back to step 3 and increase the order. Otherwise, go to step 6.

Step6. From the $N$ sets of extracted parameters, calculate $\mu, \sigma$, and $\rho$. These values represent the statistical properties of the mapping parameters $\phi$. Step7: $\quad$ Based on the calculated values of $\mu, \sigma$, and $\rho$, regenerate another set of mapping parameters $\phi$ of size $M$ used for statistical validation test, which usually larger than $N$. If the hypothesis tests error of DC and S parameters of the new device population $M$ does not meet the statistical test accuracy requirement, go back to step 4 and increase the sample number; else prepare to go to large-signal test.

Step 8. Combine the nominal model and the mapping to form the large-signal statistical model as shown in Fig. 3.3. The final statistical linear dynamic space mapped model can then be used for large-signal statistical design.

In general, once the proper statistical parameters extraction is completed, check all the hypothesis tests fit both the optimization error test and statistical validation test, the statistical linear dynamic space mapped model then is able to reproduce the 
statistical behavior of the entire device population based on the given the $\mu, \sigma$, and correlation matrix $\rho$. Finally, the model could be incorporated into existing circuit simulators for large-signal statistical design and yield estimation. Fig.3.4 shows the flow chart of the entire procedure.

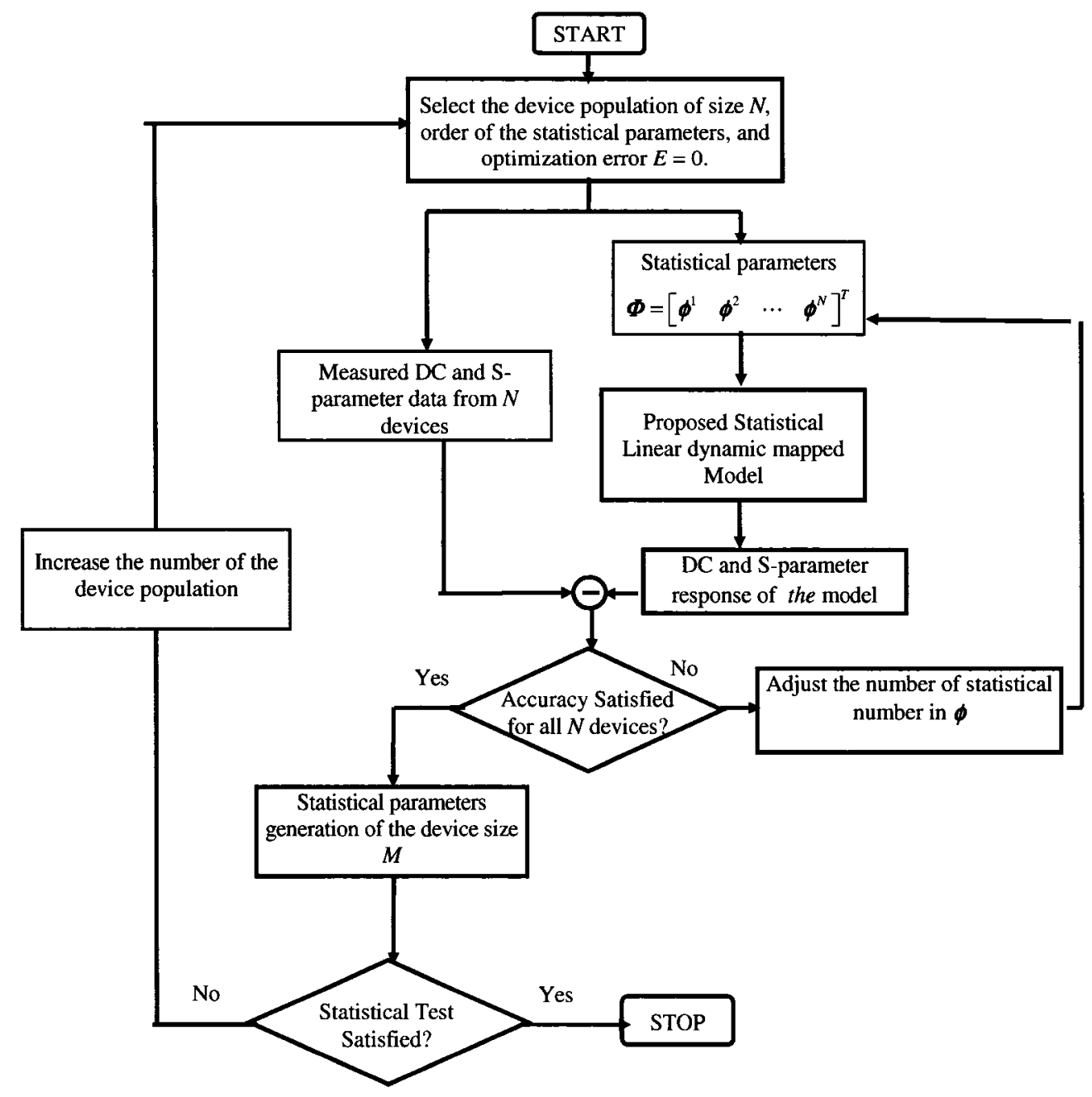

Figure 3.4 Flow chart of linear dynamic space mapping for large-signal statistical modeling. 


\subsection{Objective of the Optimization for Statistical Linear Dynamic Space Mapping Model}

The statistical linear dynamic space mapped model will not be able to accurately represent the true statistics of the device population unless the extracted statistical parameters are properly trained through the optimization from the device population. This is because the later calculated means $(\mu)$, standard deviations $(\sigma)$, and the correlation coefficients $(\rho)$ of $\Phi$ are going to be used to estimate the statistical information for the entire device population. Frankly, the key of this whole statistical modeling process is based on how good the optimization is, this means that the objective of the proposed optimization is to maximally minimize the total difference $E$ of DC and small-signal between the statistical models and actual devices in the population:

$$
\min \left(\frac{1}{2} \sum_{k=1}^{N} \sum_{j=1}^{N_{s}}\left\|y_{\text {sati }}\left(\boldsymbol{\phi}^{k}, v_{j}\right)-\boldsymbol{y}_{j}^{k}\right\|^{2}\right)
$$

where $y\left(\phi^{k}, v_{j}\right)$ is a vector representing the DC or S-parameters responses of the $k^{\text {th }}$ $(k=1,2, \ldots, N)$ statistical linear dynamic model evaluated at the $j^{\text {th }}$ input sample $v_{j}$, and $y_{j}^{k}$ is the corresponding data from the $k^{\text {th }}$ device sample in the given population. $N$ is the number of devices in the statistical population, and $N_{s}$ is the number of bias and frequency combinations for each device. 


\section{Requirements for DC and S-parameter Measurement}

The proposed statistical linear dynamic mapping technique has limited the complete large-signal measurement only to one nominal device, thus dramatically reducing the cost of expensive large-signal measurement for all device samples in the statistical population; however, the rest of the devices are still need to be measured in DC and S-parameters. To further cut down the cost for DC and Sparameter measurement for the rest devices, selection of necessary bias and frequency is very important.

\section{Bias selection}

Choose about 6-12 points along the nominal load line including the quiescent bias. The bias points should be able to cover the overall required region of the nominal device, i.e., $V_{g}$ is selected from the Current-Voltage plot where the drain current $I_{d}$ sits between $\left(I_{d \min }, I_{d \max }\right)$, and $V_{d}$ is selected between $\left(V_{d \min }, V_{d \max }\right)$. Define this bias set as $\boldsymbol{B P}_{\text {nom }}$. Choose 6-12 biases above the load line and 6-12 points below the load line. Define these two bias sets as $\boldsymbol{B} \boldsymbol{P}_{\text {shift. }}$

\section{Frequency selection}

Given center frequency $f_{c}$ and bandwidth $B W$, Choose 10 to 20 frequency points from $f_{\text {lower }}-B W$ to $f_{\text {upper }}+B W$, and another 10 to 20 points from $f_{\text {upper }}+B W$ to $N_{H} \cdot f_{\text {upper }}$, where $f_{\text {lower }}=f_{c}-0.5 \mathrm{BW}$, and $f_{\text {upper }}=f_{c}+0.5 \mathrm{BW} \cdot N_{H}$ is the number of harmonics for large-signal operation. Define $\boldsymbol{F}_{\text {meas }}$ including these frequency points as the measurement frequency set. 


\section{DC measurement}

Perform DC I-V measurement for the nominal device at the bias point $B P \in\left[\begin{array}{ll}\boldsymbol{B P}_{\text {nom }} & \boldsymbol{B} \boldsymbol{P}_{\text {shiff }}\end{array}\right]$. Perform DC I-V measurement for the rest of the devices in the device population $\boldsymbol{D}$ at $B P \in \boldsymbol{B} \boldsymbol{P}_{n m}$.

\section{S-parameter Measurement}

Perform small-signal S-parameter measurement for the nominal device at bias $B \boldsymbol{B} \in\left[\begin{array}{ll}\boldsymbol{B P}_{\text {nom }} & \boldsymbol{B P}_{\text {shif }}\end{array}\right]$ and frequency freq $\in \boldsymbol{F}_{\text {meas }}$. Perform small-signal S-parameter measurement for the rest of devices in the device population $\boldsymbol{D}$ at $B P \in \boldsymbol{B P}_{\text {nom }}$ and freq $\in \boldsymbol{F}_{\text {meas }}$.

\section{Data generation}

To verify the proposed statistical linear dynamic space mapped modeling technique, DC and S-parameter data for two different device populations are measured following the proposed measurement algorithm. One set is for statistical parameter extraction and the other set is used to validate the statistical performance of the proposed model.

\section{Order selection}

Another factor that affects the accuracy of the statistical model is the number of statistical parameters. A natural guess for the number of the statistical parameters of the proposed model is that of the dominant factors in the fabrication process. A 
large number of statistical parameters may results in highly correlated elements in $\phi$. On the other hand, few statistical parameters may not be adequate to accurately represent the statistical effects in the given device population.

\section{Evaluation of the Statistical Linear Dynamic Space Mapped Model}

The equivalence of statistical distribution between two populations of proposed models and devices is generally validated by the hypothesis test [3][52]. Based on the assumption of small variation of the statistical parameters, we feel lucky to have the outputs also fall into Gaussian distribution. Here we formulate simpler average error criteria to check the accuracy of means and variances between model and device populations as

$$
\begin{aligned}
& E_{\mu}=\frac{1}{N_{y} \cdot N_{s}} \cdot \sum_{i=1}^{N_{y}} \sum_{j=1}^{N_{s}}\left|\frac{\mu_{y_{j j}}-\mu_{y_{d_{i j}}}}{\mu_{y_{d_{j j}}}}\right| \\
& E_{\sigma}=\frac{1}{N_{y} \cdot N_{s}} \cdot \sum_{i=1}^{N_{y}} \sum_{j=1}^{N_{s}}\left|\frac{\sigma_{y_{j i}}-\sigma_{y_{d_{i j}}}}{\sigma_{y_{d_{j j}}}}\right|
\end{aligned}
$$

where $E_{\mu}$ and $E_{\sigma}$ represent the average error of $\boldsymbol{\mu}$ and $\sigma$, respectively. $N_{y}$ represents the number of outputs and $N_{s}$ is the number of training samples for each device. $N_{y}$ equals 9 for DC and small-signal design where the outputs include DC and real and imaginary parts of S-parameters, and $N_{y}$ equals $1+2 N_{H}$ for large-signal HB response of up to $N_{H}$ harmonics. $N_{s}$ is the number of bias and frequency combinations for DC 
and small-signal responses, or input power level and RF frequency combinations for large-signal $\mathrm{HB}$ responses. $\mu_{y_{i_{i j}}}\left(\sigma_{y_{i_{i j}}}\right)$ and $\mu_{y_{d_{i j}}}\left(\sigma_{y_{d_{i j}}}\right)$ are the mean (standard deviation) of the $i^{\text {th }}$ output response of $j^{\text {th }}$ sample obtained from the statistical linear model and device populations, respectively.

$E_{\mu}$ and $E_{\sigma}$ are the approximate for measuring the accuracy of the proposed statistical linear model. Small $E_{\mu}$ and $E_{\sigma}$ shows good tendency match in the statistical behavior between the proposed statistical model population and the real device population.

To evaluate the real accuracy of the statistics in the proposed model population, the hypothesis test is introduced; the error function is formulated based on the cumulative probability distribution [2] of the two populations. Let $\boldsymbol{Y}=\left[\begin{array}{llll}Y_{1} & Y_{2} & \cdots & Y_{N}\end{array}\right]^{T}$ be a vector containing $N$ statistical samples of a single output $y$. Define the cumulative probability distribution of $\boldsymbol{Y}$ as

$$
C(y)=\frac{n_{y}}{N}
$$

where $n_{y}$ is the number of data points in $\boldsymbol{Y}$ which are smaller than or equal to $y$. Let $\boldsymbol{Y}_{d}=\left[\begin{array}{llll}Y_{d_{1}} & Y_{d_{2}} & \cdots & Y_{d_{N_{d}}}\end{array}\right]^{T}$ be the output samples of size $N_{d}$ from device population, and similarly $\boldsymbol{Y}_{m}=\left[\begin{array}{llll}Y_{m_{1}} & Y_{m_{2}} & \cdots & Y_{m_{N_{m}}}\end{array}\right]^{T}$ be the output samples of size $N_{m}$ from the model population. The corresponding cumulative probability distributions of these two samples are evaluated by (3.6) as $C_{d}(y)$ and $C_{m}(y)$, respectively. The matching error 
is defined as the absolute value of the area between the two cumulative probability distributions $C_{d}(y)$ and $C_{m}(y)$ :

$$
e_{y}=\int_{-\infty}^{+\infty}\left|C_{d}(y)-C_{m}(y)\right| d y
$$

Equation (3.7) provides the measure of the accuracy of the statistics in the proposed statistical linear model. The output $y$ could be S-parameters for small-signal simulation, or power gain and intermodulation distortion for large-signal harmonic balance (HB) simulation.

\subsection{Summary}

Based on the existing advanced modeling theory, i.e. Neural-SM, linear dynamic space mapping for large-signal statistical modeling of nonlinear devices is proposed. The proposed technique utilizes the concept from the knowledge-based method, Neural-SM technique and replaces the neural network by a simpler set of linear dynamic space mapping functions.

Linear dynamic space mapping technique also extends the application into the large-signal statistical modeling. Instead of using the weights in the neural network as the statistical parameters, the coefficients of the linear dynamic functions are used. The application of this new proposed technique is limited under the assumption that the physical or geometrical variation of the device population 
should be small, thus the variation of DC and S-parameter could be small enough to be modeled as in Gaussian distribution. 


\section{Chapter 4}

\section{Application Examples of Linear Dynamic}

\section{Space Mapping Technique}

\subsection{MESFET Statistical Modeling}

To demonstrate the proposed statistical modeling technique, we first examine the statistical behavior of a nonlinear population of 100 devices, which is represented by an internal MESFET [50] in ADS [53]. For the illustration purpose, five equivalent circuit parameters, $a_{2}, r, C_{g s}, C_{g d}$, and $C_{d s}$, are considered as the physical or geometrical variation effect on these equivalent parameters in the Curtice model and perturbed around the given mean values with certain average standard deviation for these parameters. Specified by the average standard deviations, three modeling cases are performed of $+/-3 \%,+/-5 \%$, and $+/-10 \%$. The nominal model in this example is the MESFET model whose parameters are exactly the mean values shown in table 4.1 below. The average $+/-5 \%$ standard deviation case will be used as the detailed example here. 
Table 4.1

Mean/Nominal Values and Equivalent Parameter Standard Deviation Change for MESFET Example

\begin{tabular}{|c|c|c|c|}
\hline Mean Values & $\begin{array}{l}\text { Average 3\% } \\
\text { Standard } \\
\text { deviations }\end{array}$ & $\begin{array}{l}\text { Average 5\% } \\
\text { Standard } \\
\text { deviations }\end{array}$ & $\begin{array}{l}\text { Average 10\% } \\
\text { Standard } \\
\text { deviations }\end{array}$ \\
\hline$a_{2}: 0.0143117$ & 0.0005 & 0.001 & 0.002 \\
\hline$r: 1.72208$ & 0.025 & 0.05 & 0.10 \\
\hline$C_{g s:} 3.21539 \mathrm{e}-13$ & 0.10 & 0.20 & 0.40 \\
\hline$C_{g d:} 2.32076 \mathrm{e}-13$ & 0.075 & 0.15 & 0.30 \\
\hline$C_{d s:} 2.53698 \mathrm{e}-13$ & 0.075 & 0.15 & 0.30 \\
\hline
\end{tabular}

According to the previous chapter, DC and S-parameter data are generated by $A D S$ simulation at 40 frequencies $(800 \mathrm{MHz} \sim 20 \mathrm{GHz})$ and 11 bias points across the load line. $\mathrm{Vg}$ is in the range of $[-1.4 \mathrm{v}, 0.6 \mathrm{v}]$ and $\mathrm{Vd}$ is in the range of $[0.4 \mathrm{v}, 5.3 \mathrm{v}]$. The linear dynamic order is selected for 0 , which means there is no derivative of $\mathrm{Vg}$ or Vd used. There are total 6 statistical parameters.

Once the extraction of the statistical parameters is done by the optimization, the optimization error is performed by the tests of mean, standard deviation, and hypothesis. Mean and standard deviation tests are used for the rough approximation comparison and also the hypothesis tests, Kolmogorov-Smirnov (K-S) goodness-offit test [2], is used to compare the empirical cumulative distribution function (ECDF) of the two populations, model and device. The errors are $0.49 \%, 41.43 \%$, and $4.16 \%$ respectively, which are considerably small. 
Based on the mean, standard deviation, and correlation, statistical parameters are regenerated again and used in statistical accuracy test, shown in tables 4.2-4.5. The well-constructed statistical linear dynamic model is implemented into $A D S$ [53] for performance test. Figs. 4.1 and 4.2 show the comparison of the mean, standard deviation, and ECDF of S-parameter statistics of 250 Monte-Carlo analyses using the original ADS MESFET and the proposed model. The results from these two figures show that our proposed model has achieved relatively good accuracy. After training, the proposed statistical linear dynamic model can approximate the statistical variation in the DC and small-signal behavior of the device population. The overall statistical model is further tested for 100 Monte-Carlo analyses of twotone harmonic balance (HB) simulation. Comparisons between model solution and original response are shown in Fig. 4.3-4.5 for the third order interception (TOI), power added efficiency (PAE), and power gain. All results show that the largesignal responses from the model and the device population have a good match for mean value and reasonable approximation for standard deviation. This confirms that the proposed statistical linear dynamic space mapping model extracted from DC and small-signal data can exceed its capability for large-signal statistical design and yield estimation.

Table 4.2

Mean of Statistical Parameters Before and After Parameter Regeneration

\begin{tabular}{|l|l|l|l|l|l|l|}
\hline Mean & Para \#1 & Para \#2 & \multicolumn{1}{|l|}{ Para \#3 } & \multicolumn{1}{|l|}{ Para \#4 } & \multicolumn{1}{c|}{ Para \#5 } & \multicolumn{1}{c|}{ Para \#6 } \\
\hline $\begin{array}{l}\text { Extracted } \\
\text { parameters }\end{array}$ & 0.99889 & -0.00017 & -0.00053 & 0.001714 & 1.0042 & -0.00092 \\
\hline $\begin{array}{l}\text { Regenerated } \\
\text { parameters }\end{array}$ & 0.99889 & -0.00017 & -0.00053 & 0.001714 & 1.0042 & -0.00092 \\
\hline
\end{tabular}


Table 4.3

Standard Deviation of Statistical Parameters Before and After Parameter Regeneration

\begin{tabular}{|l|l|l|l|l|l|l|}
\hline $\begin{array}{l}\text { Standard } \\
\text { Deviation }\end{array}$ & Para \#1 & Para \#2 & Para \#3 & Para \#4 & Para \#5 & Para \#6 \\
\hline $\begin{array}{l}\text { Extracted } \\
\text { parameters }\end{array}$ & 0.011471 & 0.002094 & 0.005558 & 0.021472 & 0.046448 & 0.034022 \\
\hline $\begin{array}{l}\text { Regenerated } \\
\text { parameters }\end{array}$ & 0.011286 & 0.00206 & 0.005484 & 0.021142 & 0.046134 & 0.033485 \\
\hline
\end{tabular}

Table 4.4

Correlation Coefficients of Statistical Parameters Before Parameter Regeneration

\begin{tabular}{|r|r|r|r|r|r|}
\hline 1 & & & & & \\
\hline 0.96672 & 1 & & & & \\
\hline 0.28616 & 0.13777 & 1 & & & \\
\hline 0.18675 & 0.4233 & -0.36119 & 1 & & \\
\hline 0.48884 & 0.6481 & -0.23763 & 0.76819 & 1 & \\
\hline-0.46785 & -0.67016 & 0.2091 & -0.95432 & -0.83805 & 1 \\
\hline
\end{tabular}

Table 4.5

Correlation Coefficients of Statistical Parameters After Parameter Regeneration

\begin{tabular}{|r|r|r|r|r|r|}
\hline 1 & & & & & \\
\hline 0.9667 & 1 & & & & \\
\hline 0.28435 & 0.13487 & 1 & & & \\
\hline 0.18887 & 0.42518 & -0.36156 & 1 & & \\
\hline 0.49355 & 0.65209 & -0.252 & 0.76839 & 1 & \\
\hline-0.46879 & -0.6709 & 0.21094 & -0.95465 & -0.83843 & 1 \\
\hline
\end{tabular}



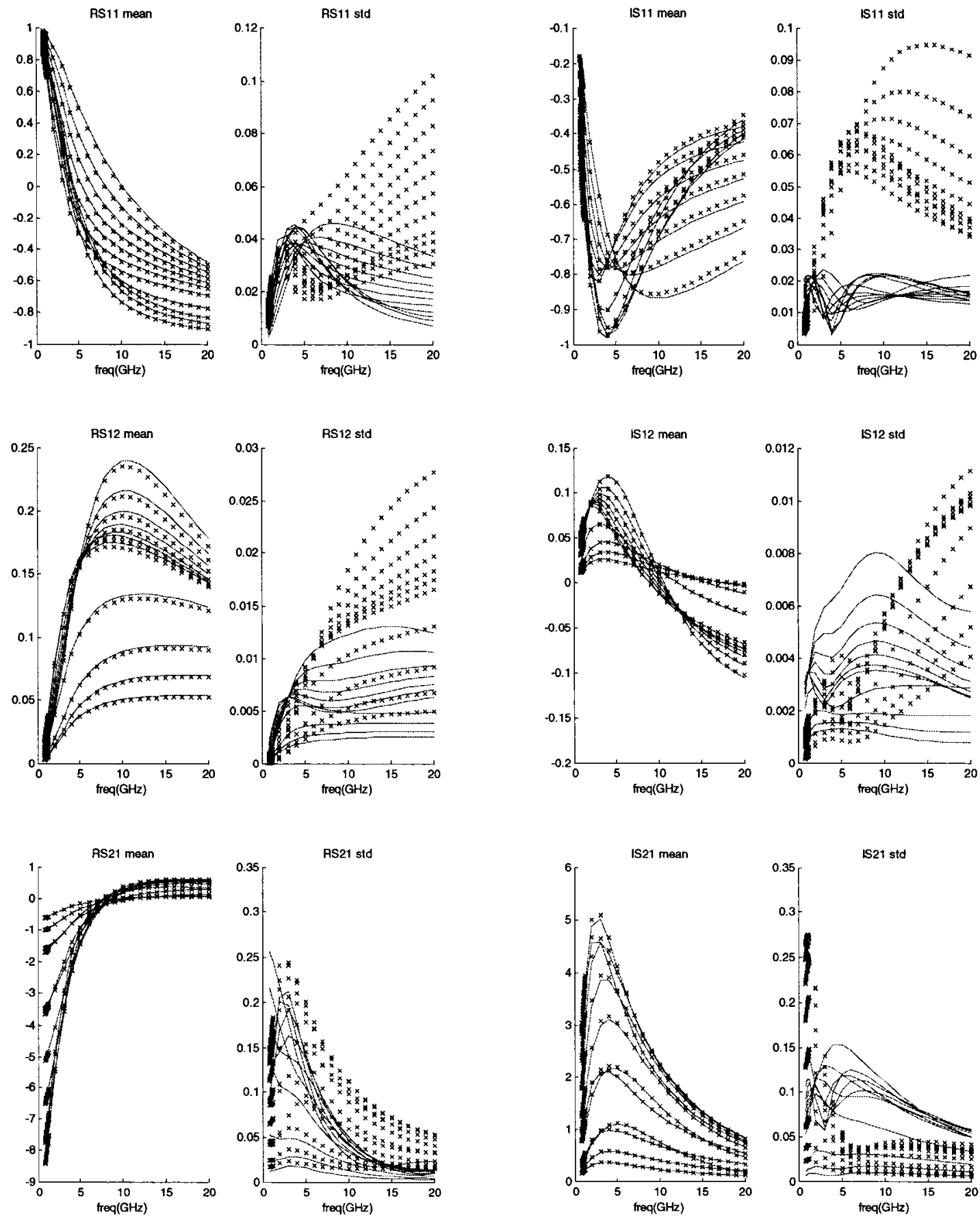

Figure 4.1 Comparison of mean value $(\mu)$ and standard deviation $(\sigma)$ of real and imaginary parts of S-parameters between 250 MESFET devices (-) and 250 proposed statistical linear dynamic space mapping models $(x)$. Error of mean is $0.62 \%$ and error of standard deviation is $67.44 \%$ 

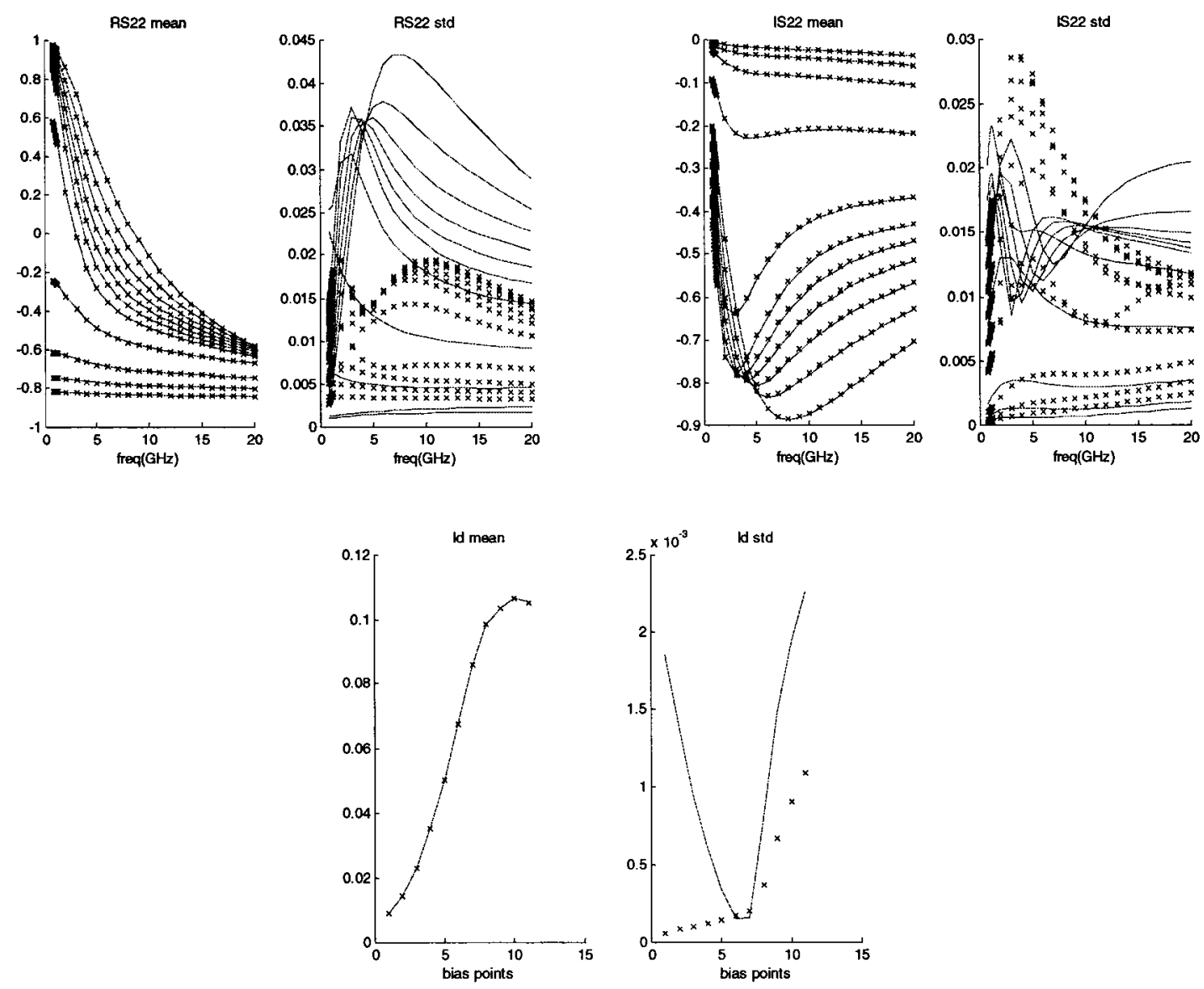

Figure 4.1 (Continued). 


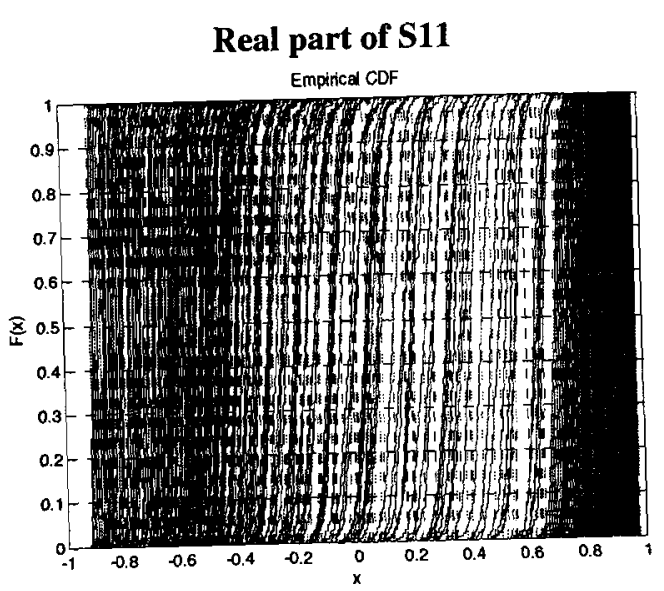

Real part of $\mathrm{S} 12$

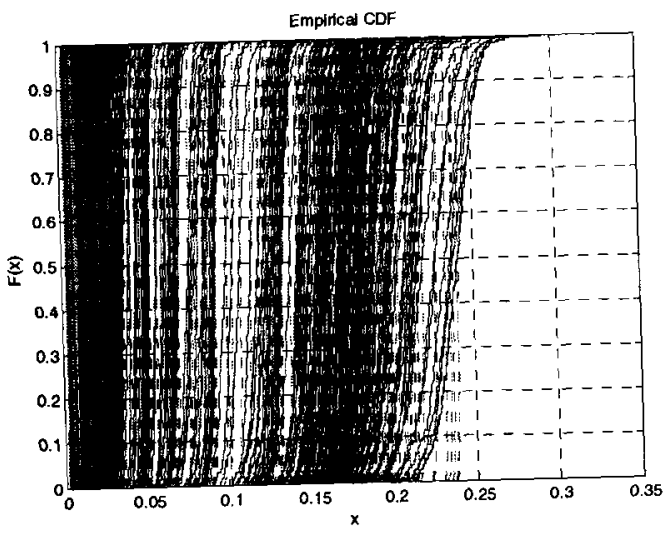

Real part of $S 21$

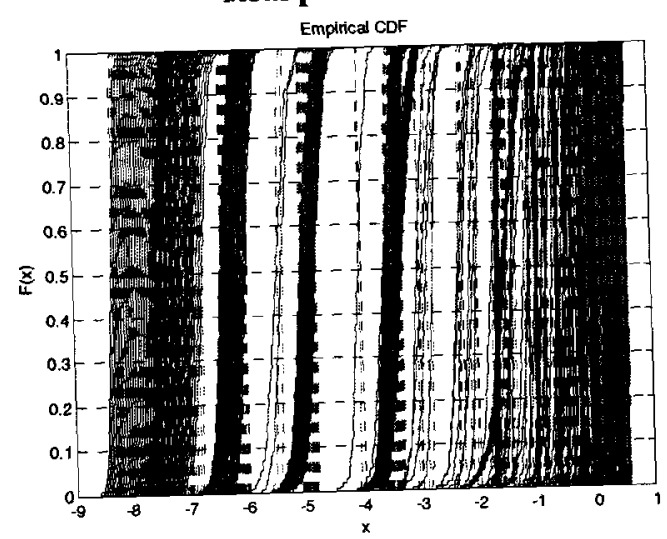

Imaginary part of S11

Empincal CDF

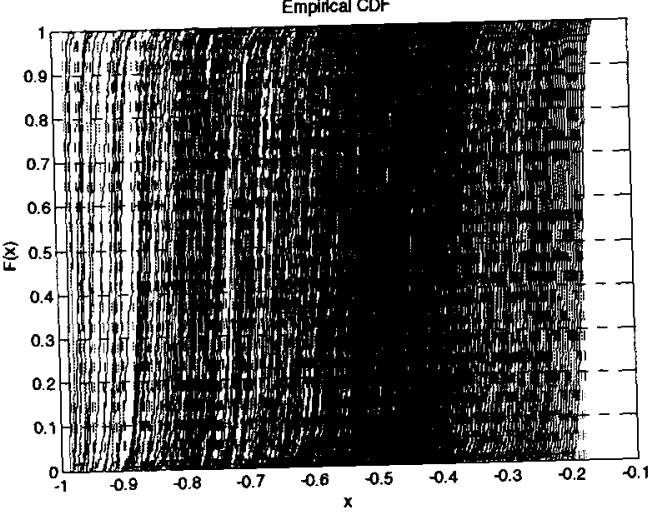

Imaginary part of S12

Empincal CDF

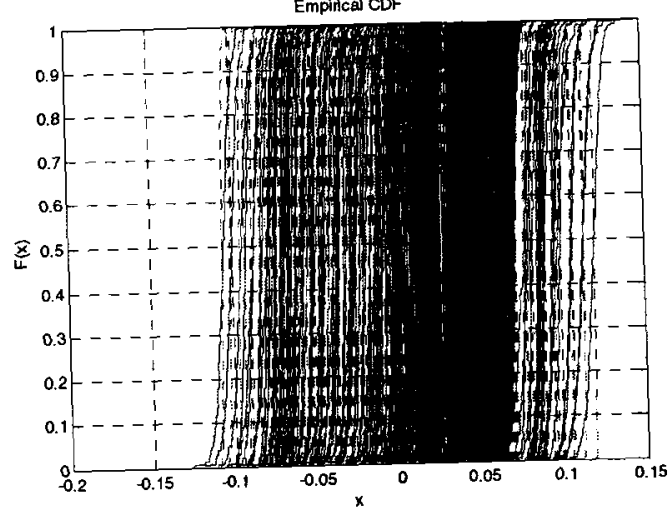

Imaginary part of $S 21$

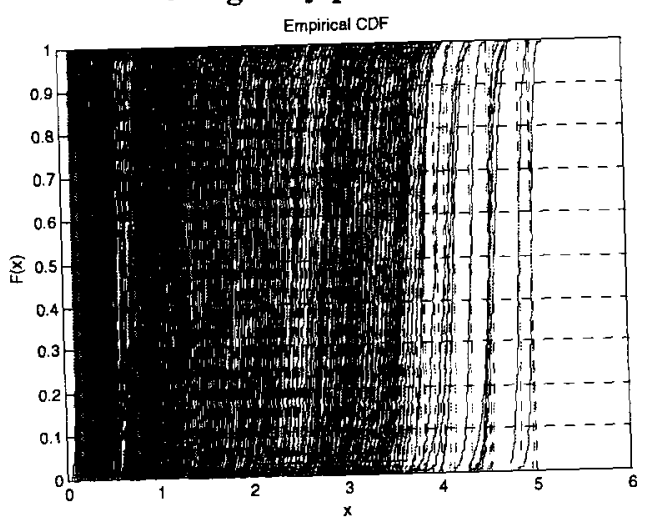

Figure 4.2 Comparison of ECDF of DC and S-parameters in real and imaginary parts between 250 MESFET devices (dash line) and 250 proposed statistical linear dynamic space mapping models (solid line). Overall error is $6.47 \%$. 

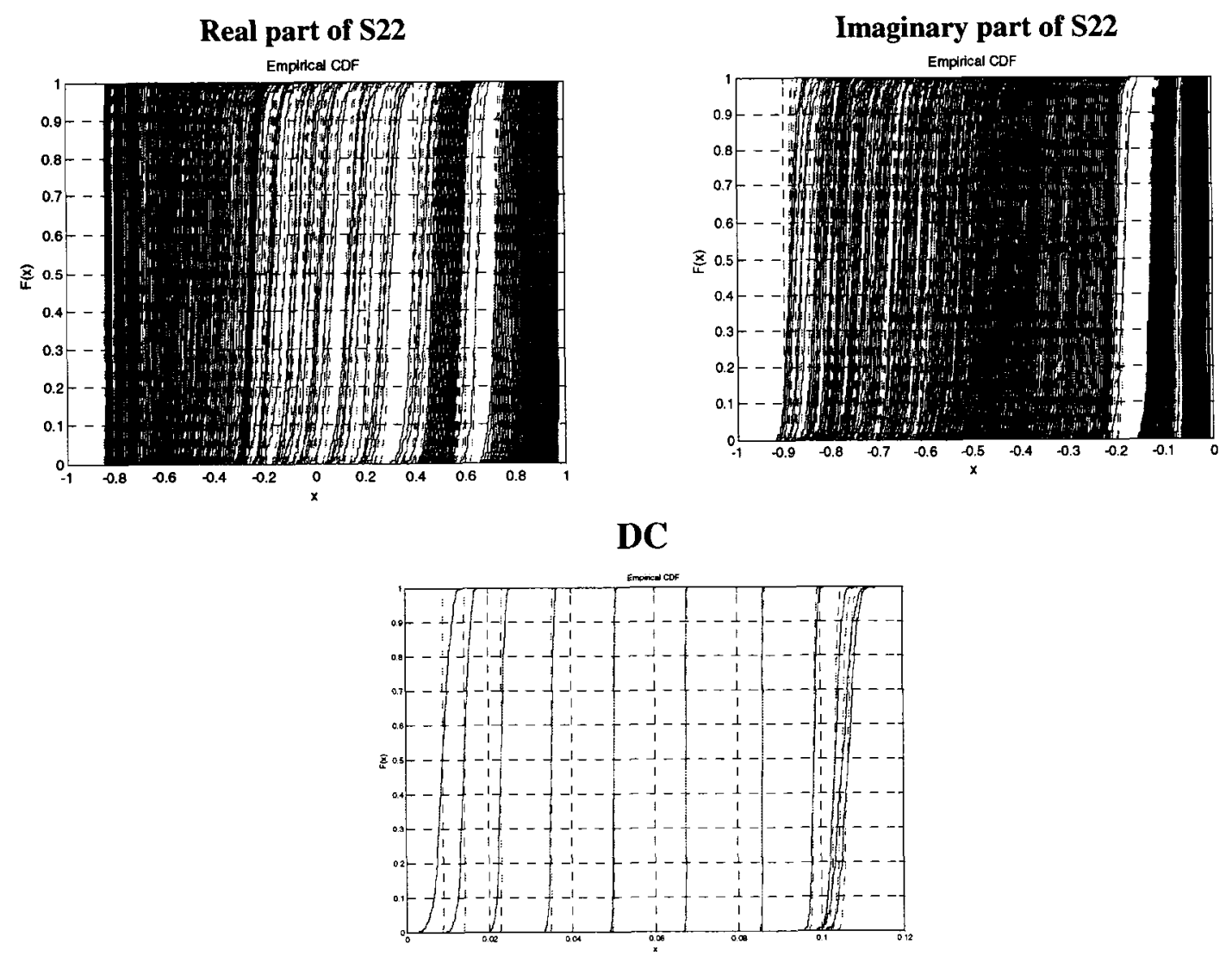

Figure 4.2 (Continued). 

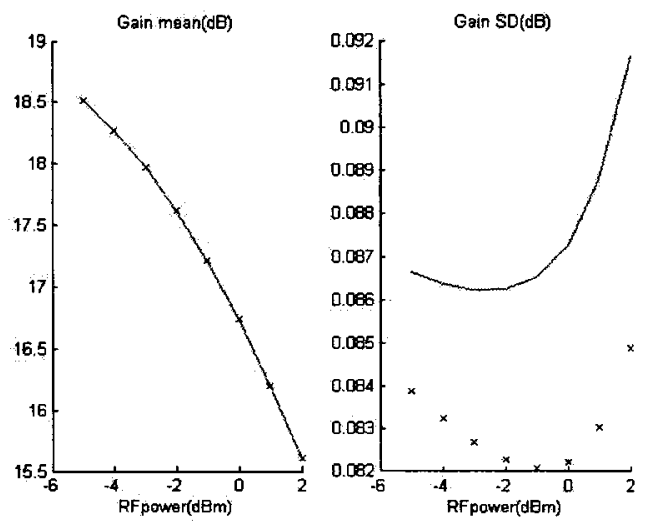

(a)
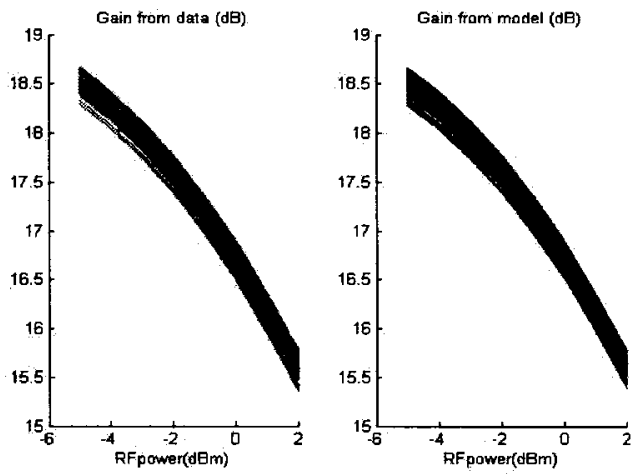

(b)

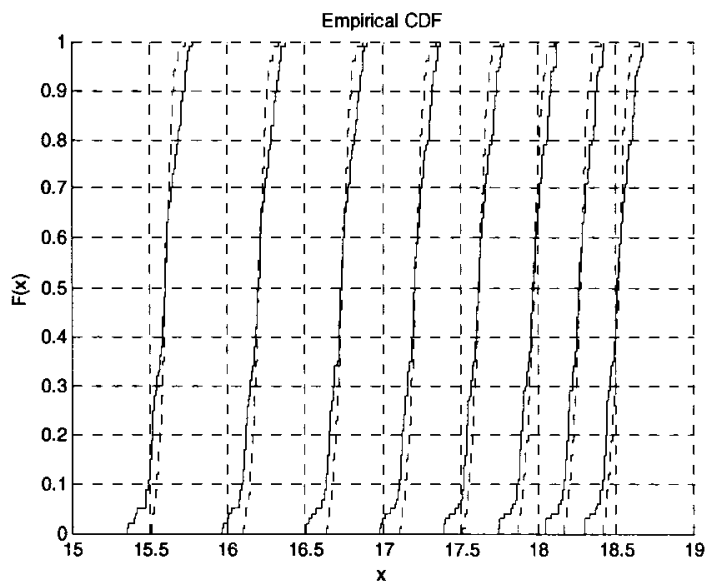

(c)

Figure 4.3 Power Gain Comparison between 100 statistical models and 100 devices: (a) Mean and standard deviation (b) Distribution (c) ECDF - device in dash line and model in solid line. 

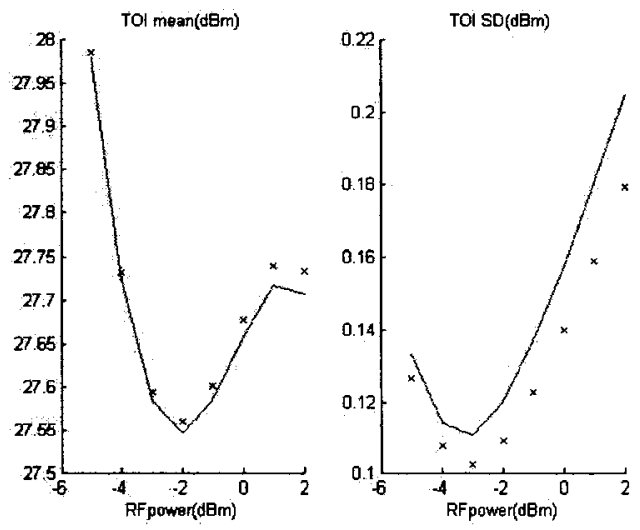

(a)
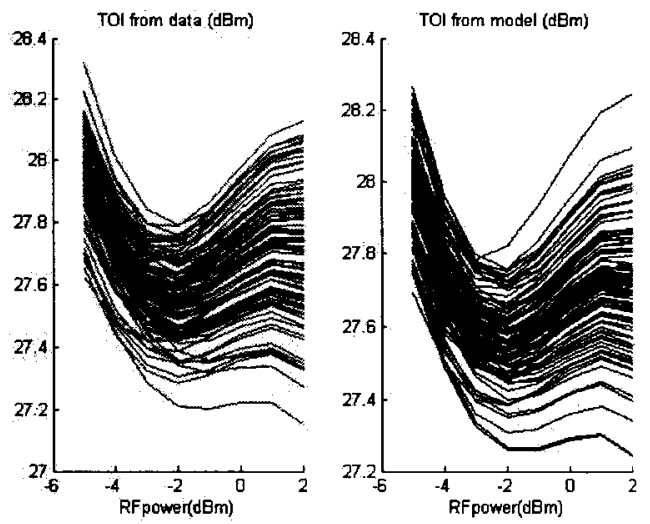

(b)

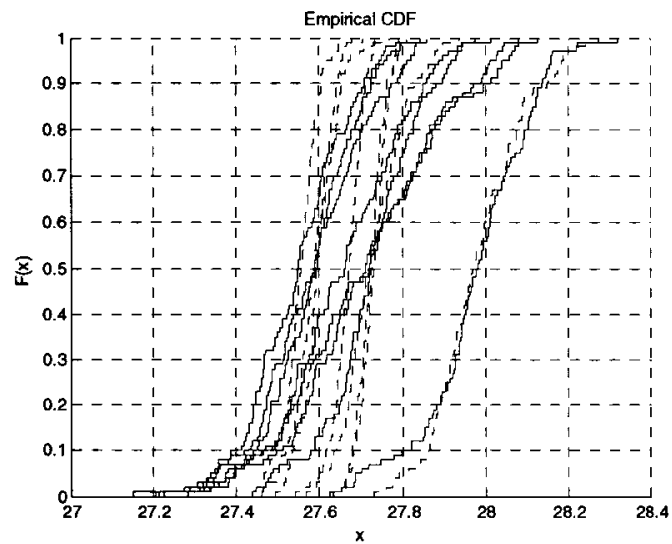

(c)

Figure 4.4 TOI Comparison between 100 statistical models and 100 devices: (a) Mean and standard deviation (b) Distribution (c) ECDF - device in dash line and model in solid line. 

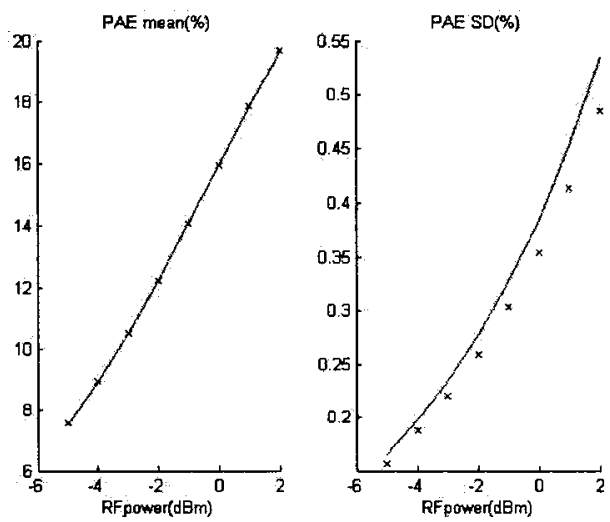

(a)
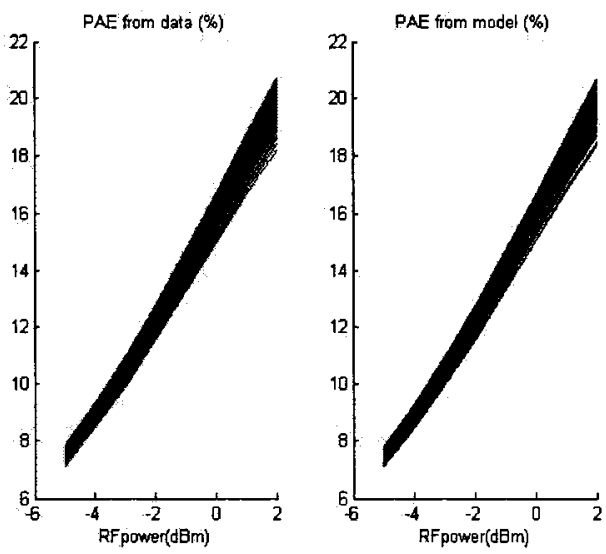

(b)

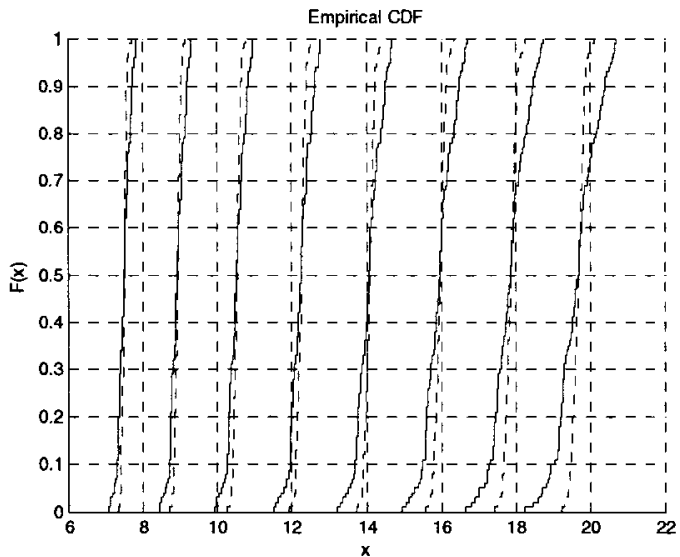

(c)

Figure 4.5 PAE Comparison between 100 statistical models and 100 devices: (a) Mean and standard deviation (b) Distribution (c) ECDF - device in dash line and model in solid line. 
Table 4.6

Comparison Between the Device and the Statistical Linear Dynamic Mapping Model.

\begin{tabular}{|c|c|c|c|c|c|}
\hline \multicolumn{6}{|c|}{ Linear Dynamic Space Mapping Model Testing Results } \\
\hline $\begin{array}{l}\text { Extraction } \\
\text { accuracy }\end{array}$ & $\begin{array}{l}\text { Order } \\
\text { selection }\end{array}$ & Comparison & $\begin{array}{l}\text { Error of } \\
\text { mean } \%\end{array}$ & $\begin{array}{l}\text { Error of } \\
\text { SD \% }\end{array}$ & $\begin{array}{l}\text { Error of } \\
\text { ECDF \% }\end{array}$ \\
\hline \multirow[t]{4}{*}{$3.0 \%$} & \multirow[t]{4}{*}{4 variables } & $\mathrm{DC}$ and $\mathrm{S}$ & 0.25 & 43.24 & 4.61 \\
\hline & & TOI & 0.03 & 16.07 & 8.57 \\
\hline & & PAE & 0.019 & 38.66 & 4.96 \\
\hline & & Gain & 0.12 & 25.97 & 5.19 \\
\hline \multirow[t]{4}{*}{$5.0 \%$} & \multirow[t]{4}{*}{6 variables } & $\mathrm{DC}$ and $\mathrm{S}$ & 0.62 & 67.44 & 6.47 \\
\hline & & TOI & 0.026 & 47.12 & 9.73 \\
\hline & & PAE & 0.034 & 59.23 & 7.27 \\
\hline & & Gain & 0.095 & 62.47 & 6.34 \\
\hline \multirow[t]{4}{*}{$10 \%$} & \multirow[t]{4}{*}{12 variables } & DC and $S$ & 0.84 & 74.57 & 7.24 \\
\hline & & TOI & 0.023 & 49.82 & 12.38 \\
\hline & & PAE & 0.045 & 56.78 & 13.11 \\
\hline & & Gain & 0.078 & 63.04 & 10.54 \\
\hline
\end{tabular}

All results show that the small and large-signal responses from the model and the device population have a good match in mean, standard deviation, and ECDF comparisons. From the table 4.6, we can conclude that, when the device variation is small, the proposed technique is suitable for the statistical modeling. As the device variation increases, the proposed technique tends to have difficulties to handle; such as the PAE comparison in $10 \%$ case has the error about $13.11 \%$, which is considerably a little bit large. Overall, this example confirms that the proposed statistical linear statistical space mapping model is capable of the statistical modeling of large-signal. 


\subsection{HEMT Statistical Modeling}

This example models a High Electron Mobility Device (HEMT) process variation induced effects on electrical characteristics. The HEMT device is setup in a physics-based device simulator Synopsys Medici [54] as shown in Fig. 4.6. A large-signal nominal model [55] is extracted using Agilent IC-CAP model extraction tool [56] by performing device level simulation in Medici. Ten geometrical and physical parameters in the HEMT device are chosen as statistical parameters with $+/-3 \%,+/-5 \%$, and $+/-10 \%$ Gaussian, independent variation about their nominal values listed in Table 4.7. The average $+/-3 \%$ standard deviation case will be used as the detailed example here since this HEMT model has more nonlinear properties and harder to model compared to the MESFET example.

Following the same procedures as in MESFET example, DC and S-parameter data are generated by $A D S$ simulation at 34 frequencies $(9.8 \mathrm{GHz} \sim 50.5 \mathrm{GHz})$ and 10 bias points across the load line. $\mathrm{Vg}$ is in the range of $[0 \mathrm{v}, 0.9 \mathrm{v}]$ and $\mathrm{Vd}$ is in the range of $[2.5 \mathrm{v}, 5.15 \mathrm{v}]$. The linear dynamic order is selected for 1 , which means the first derivatives of both $\mathrm{Vg}$ and $\mathrm{Vd}$ used. There are totally 10 statistical parameters.

Again, performed by the tests of mean, standard deviation, and hypothesis, the optimization errors are $0.56 \%, 49.73 \%$, and $4.13 \%$ respectively, which are very small.

Still based on the calculated mean, standard deviation, and correlation, regenerated statistical parameters again and used in statistical accuracy test, shown in tables 4.8-4.11. The well-constructed statistical linear dynamic model is implemented into $A D S$ [53] for performance test. Figs. 4.7 and 4.8 show the 
comparison of the mean, standard deviation, and ECDF of S-parameter statistics of 250 Monte-Carlo analyses between the statistical model and the HEMT devices. The results from these two figures show that the validation test has achieved relatively good accuracy. After constructing, the proposed statistical linear dynamic model can approximate the statistical variation in the DC and small-signal behavior of the device population. The overall statistical model is further tested for 100 MonteCarlo analyses of one-tone harmonic balance (HB) simulation. Comparisons between model solution and original response are shown in Fig. 4.9-4.11 for the fundamental harmonic, second harmonic, and third harmonic.

All results show that the large-signal responses from the model and the device population have a good match in terms of mean value, standard deviation, and reasonable approximation in ECDF. This example once more confirms that the proposed statistical linear dynamic space mapping model extracted from DC and small-signal data is capability for large-signal statistical design and yield estimation. 


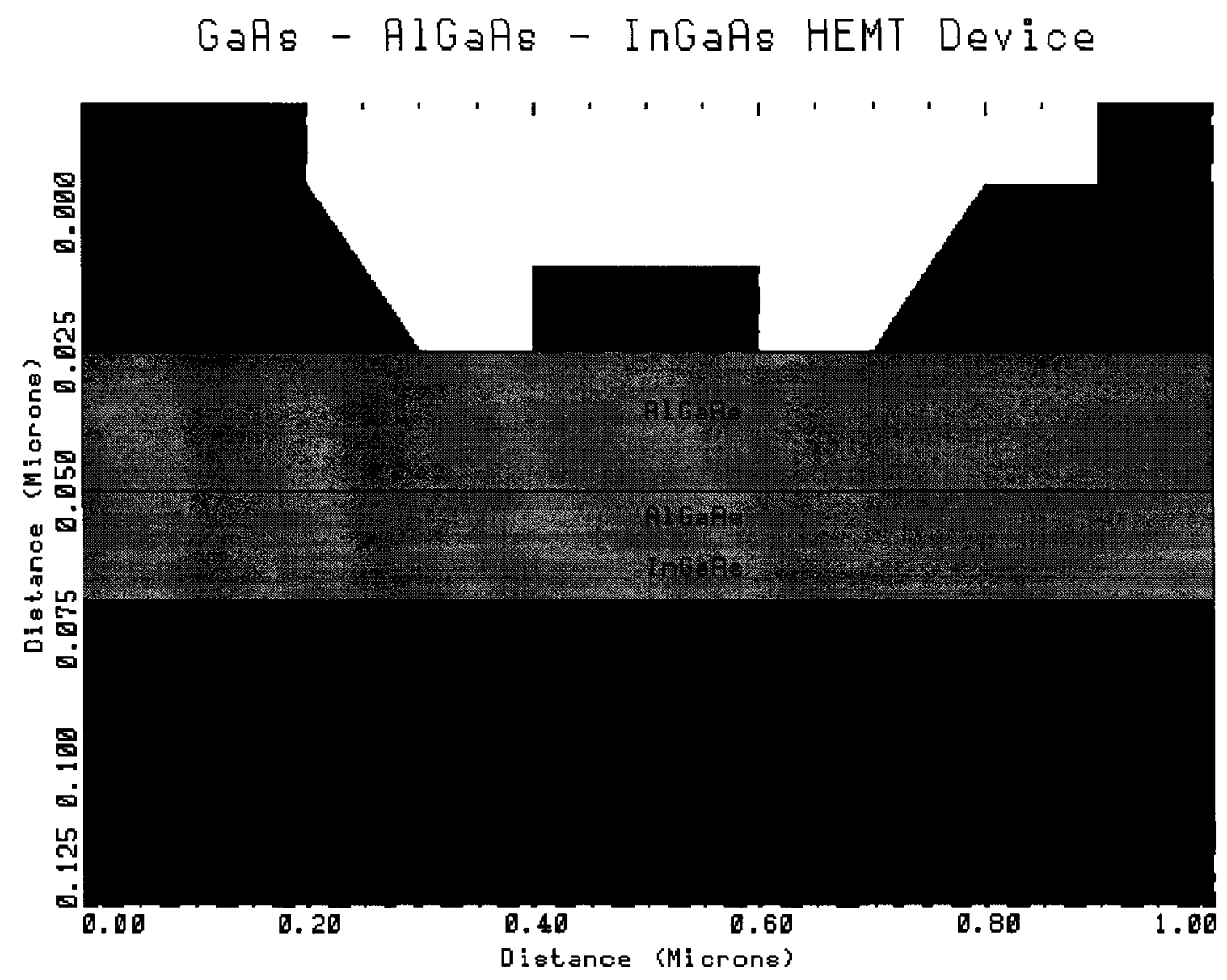

Figure 4.6 HEMT structure in Medici.

Table 4.7

Mean Values and Physical/Geometrical Parameters Change for HEMT Example

\begin{tabular}{llc}
\hline \hline Parameter Name & & MEAN $(\mu)$ \\
\hline GATE LENGTH (UM) & & 0.4 \\
Gate Width (um) & & 100 \\
& AlGaAs Donor Layer & 0.025 \\
Thickness (um) & AlGaAs Spacer Layer & 0.01 \\
& InGaAs Channel Layer & 0.01 \\
& GaAs Substrate & 0.045 \\
& InGaAs Channel Layer & $1 \mathrm{E} 2$ \\
Doping Density & AlGaAs Donor Layer & $1 \mathrm{E} 18$ \\
$\left(1 / \mathrm{m}^{3}\right)$ & Source N+ & $2 \mathrm{E} 20$ \\
& Drain N+ & $2 \mathrm{E} 20$ \\
\hline \hline
\end{tabular}


Table 4.8

Mean of Statistical Parameters Before and After Parameter Regeneration

\begin{tabular}{|l|l|l|l|l|l|l|l|l|l|l|}
\hline Mean & $\begin{array}{l}\text { Para \#1 } \\
(\mathrm{E}-1)\end{array}$ & $\begin{array}{l}\text { Para \#2 } \\
(\mathrm{E}-5)\end{array}$ & $\begin{array}{l}\text { Para \#3 } \\
(\mathrm{E}-4)\end{array}$ & $\begin{array}{l}\text { Para \#4 } \\
(\mathrm{E}-5)\end{array}$ & $\begin{array}{l}\text { Para \#5 } \\
(\mathrm{E}-3)\end{array}$ & $\begin{array}{l}\text { Para \#6 } \\
(\mathrm{E}-2)\end{array}$ & $\begin{array}{l}\text { Para \#7 } \\
(\mathrm{E}-5)\end{array}$ & $\begin{array}{l}\text { Para \#8 } \\
(\mathrm{E}-1)\end{array}$ & $\begin{array}{l}\text { Para \#9 } \\
(\mathrm{E}-5)\end{array}$ & $\begin{array}{l}\text { Para \#10 } \\
(\mathrm{E}-2)\end{array}$ \\
\hline $\begin{array}{l}\text { Extracted } \\
\text { parameters }\end{array}$ & 9.936 & 1.253 & -1.457 & 1.123 & 1.266 & -1.218 & -6.952 & 9.762 & 4.189 & 4.666 \\
\hline $\begin{array}{l}\text { Regenerated } \\
\text { parameters }\end{array}$ & 9.936 & 1.253 & -1.457 & 1.123 & 1.266 & -1.218 & -6.952 & 9.762 & 4.189 & 4.666 \\
\hline
\end{tabular}

Table 4.9

Standard Deviation of Statistical Parameters Before and After Parameter Regeneration

\begin{tabular}{|l|l|l|l|l|l|l|l|l|l|l|}
\hline $\begin{array}{l}\text { Standard } \\
\text { Deviation }\end{array}$ & $\begin{array}{l}\text { Para \#1 } \\
(\mathrm{E}-2)\end{array}$ & $\begin{array}{l}\text { Para \#2 } \\
(\mathrm{E}-4)\end{array}$ & $\begin{array}{l}\text { Para \#3 } \\
(\mathrm{E}-3)\end{array}$ & $\begin{array}{l}\text { Para \#4 } \\
(\mathrm{E}-5)\end{array}$ & $\begin{array}{l}\text { Para \#5 } \\
(\mathrm{E}-2)\end{array}$ & $\begin{array}{l}\text { Para \#6 } \\
(\mathrm{E}-1)\end{array}$ & $\begin{array}{l}\text { Para \#7 } \\
(\mathrm{E}-3)\end{array}$ & $\begin{array}{l}\text { Para \#8 } \\
(\mathrm{E}-2)\end{array}$ & $\begin{array}{l}\text { Para \#9 } \\
(\mathrm{E}-4)\end{array}$ & $\begin{array}{l}\text { Para \#10 } \\
(\mathrm{E}-1)\end{array}$ \\
\hline $\begin{array}{l}\text { Extracted } \\
\text { parameters }\end{array}$ & 3.846 & 1.573 & 2.207 & 5.534 & 1.780 & 1.394 & 1.034 & 9.698 & 2.217 & 1.066 \\
\hline $\begin{array}{l}\text { Regenerated } \\
\text { parameters }\end{array}$ & 3.785 & 1.536 & 2.182 & 5.470 & 1.758 & 1.376 & 1.012 & 9.547 & 2.193 & 1.054 \\
\hline
\end{tabular}

Table 4.10

Correlation Coefficients of Statistical Parameters Before Parameter Regeneration

\begin{tabular}{|c|c|c|c|c|c|c|c|c|c|}
\hline 1 & & & & & & & & & \\
\hline-0.83566 & 1 & & & & & & & & \\
\hline 0.704772 & -0.94877 & 1 & & & & & & & \\
\hline-0.53732 & 0.830112 & -0.81606 & 1 & & & & & & \\
\hline-0.74137 & 0.969035 & -0.99548 & 0.838087 & 1 & & & & & \\
\hline 0.896973 & -0.97614 & 0.921807 & -0.77027 & -0.94076 & 1 & & & & \\
\hline-0.74229 & 0.953047 & -0.94086 & 0.745189 & 0.9581 & -0.91837 & 1 & & & \\
\hline 0.662534 & -0.87558 & 0.853923 & -0.95209 & -0.87727 & 0.832094 & -0.77404 & 1 & & \\
\hline-0.73151 & 0.950091 & -0.91593 & 0.95614 & 0.939989 & -0.9087 & 0.878842 & -0.95391 & 1 & \\
\hline-0.33973 & 0.382737 & -0.27795 & 0.671879 & 0.315776 & -0.38285 & 0.174515 & -0.70892 & 0.546873 & 1 \\
\hline
\end{tabular}

Table 4.11

Correlation Coefficients of Statistical Parameters After Parameter Regeneration

\begin{tabular}{|r|r|r|r|r|r|r|r|r|r|}
\hline 1 & & & & & & & & \\
\hline-0.83277 & 1 & & & & & & & & \\
\hline 0.707183 & -0.95058 & 1 & & & & & & & \\
\hline-0.54081 & 0.8342 & -0.81927 & 1 & & & & & & \\
\hline-0.74272 & 0.970706 & -0.99542 & 0.841371 & 1 & & & & & \\
\hline 0.893927 & -0.97627 & 0.925958 & -0.77759 & -0.94423 & 1 & & & & \\
\hline-0.73558 & 0.951375 & -0.93826 & 0.748195 & 0.956191 & -0.91598 & 1 & & & \\
\hline 0.67581 & -0.87925 & 0.852601 & -0.9496 & -0.87641 & 0.840558 & -0.7704 & & & \\
\hline-0.72982 & 0.951227 & -0.91784 & 0.957549 & 0.941666 & -0.91131 & 0.879 & -0.95364 & & \\
\hline-0.35954 & 0.380199 & -0.2724 & 0.658426 & 0.31012 & -0.3888 & 0.162736 & -0.70555 & 0.539022 & \\
\hline
\end{tabular}



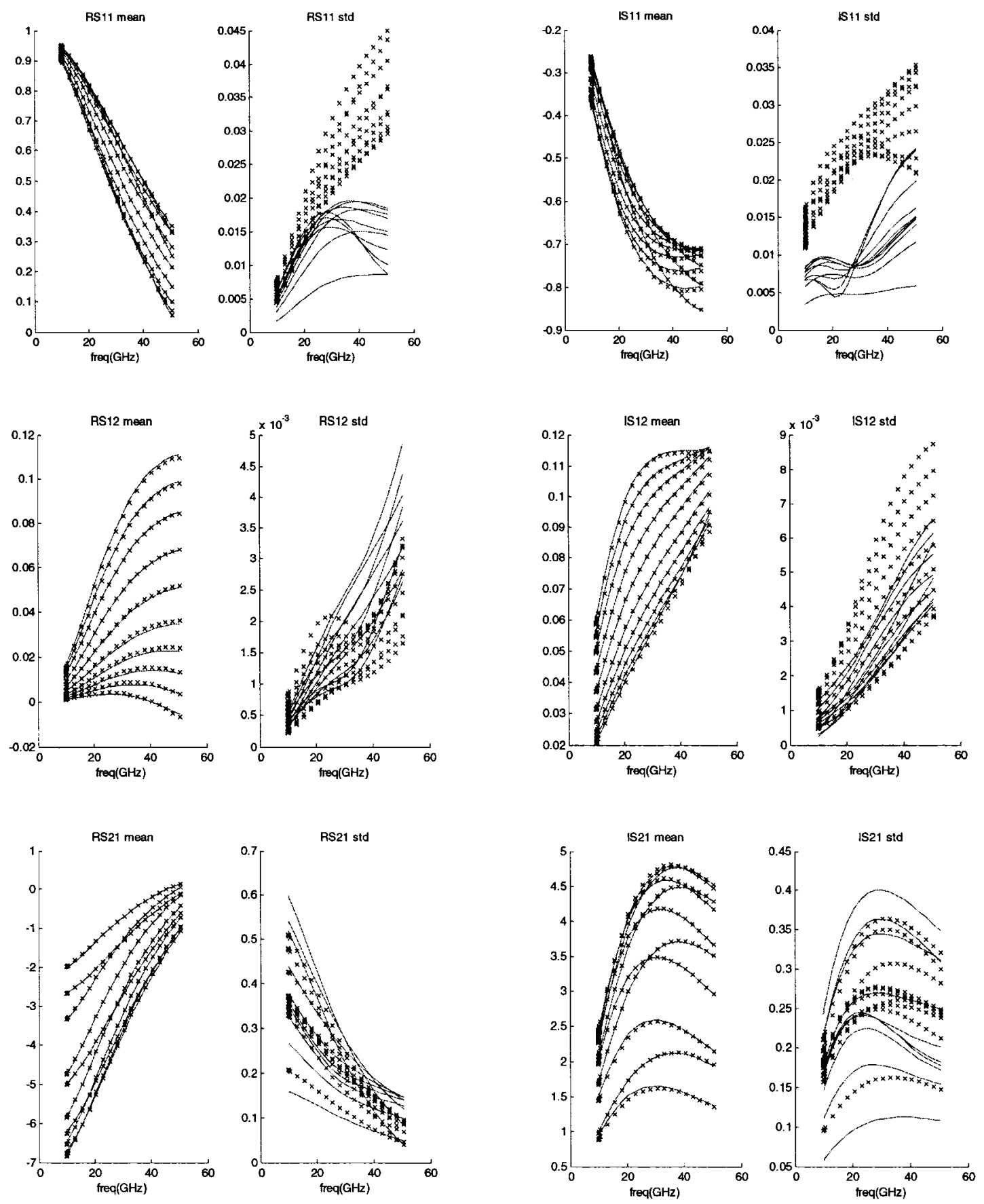

Figure 4.7 Comparison of mean value $(\mu)$ and standard deviation $(\sigma)$ of real and imaginary parts of S-parameters between 250 MESFET devices (-) and 250 proposed statistical linear dynamic space mapping models $(x)$. Error of mean is $1.64 \%$ and error of standard deviation is $\mathbf{5 1 . 0 2 \%}$ 

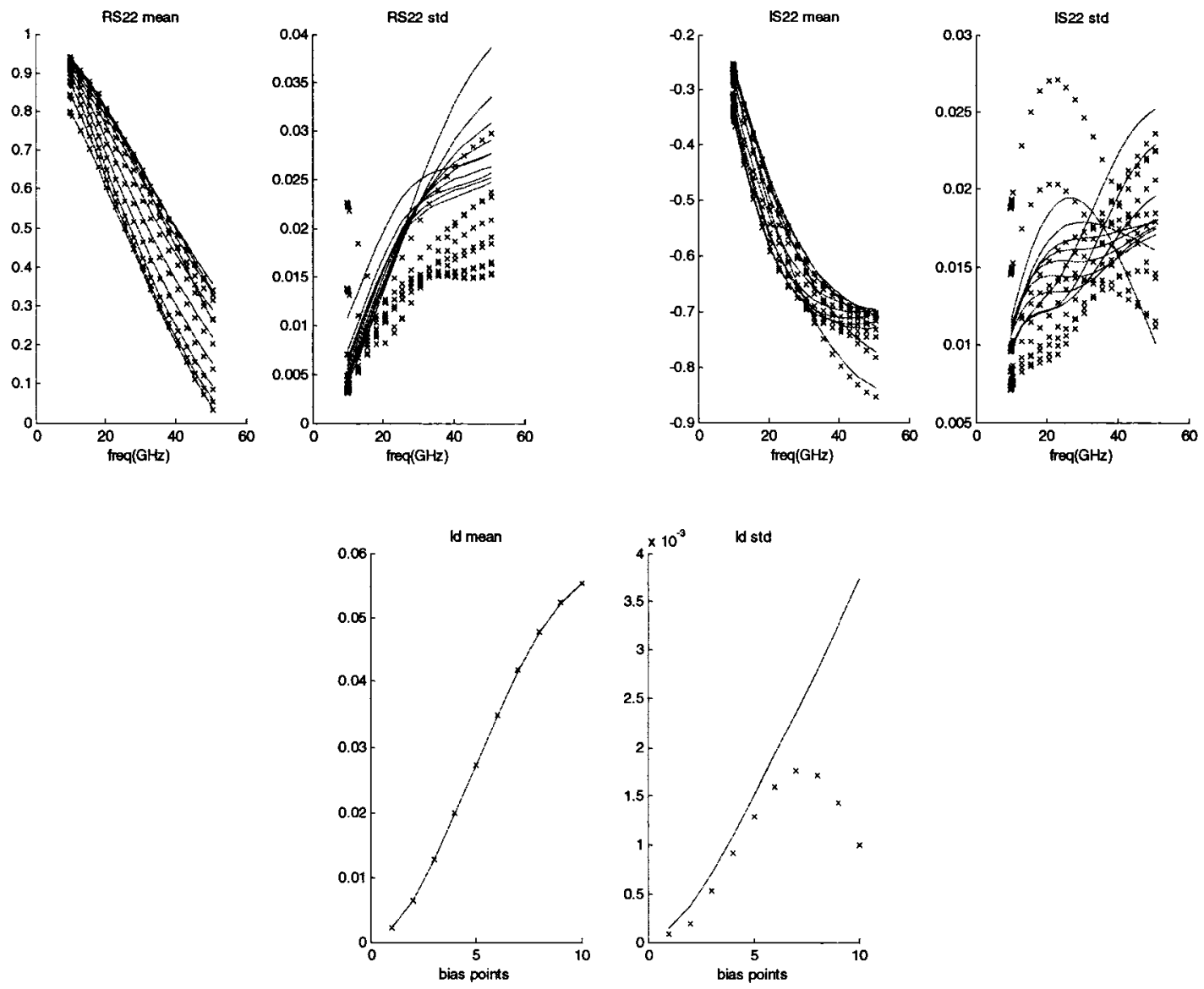

Figure 4.7 (continued). 


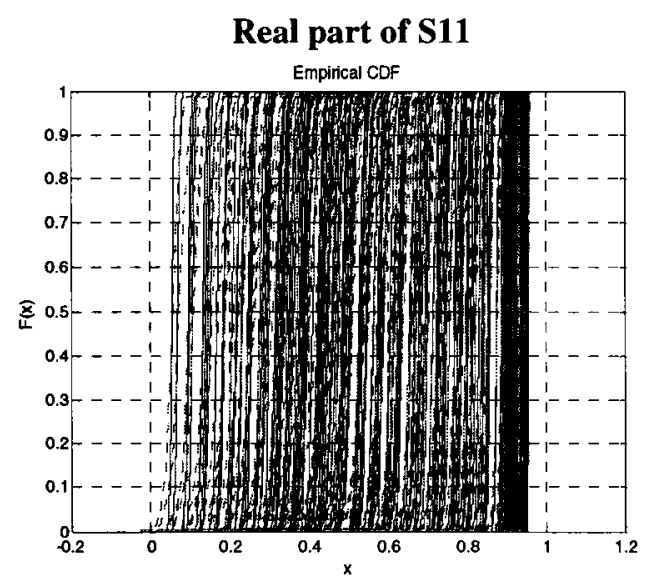

Real part of S12

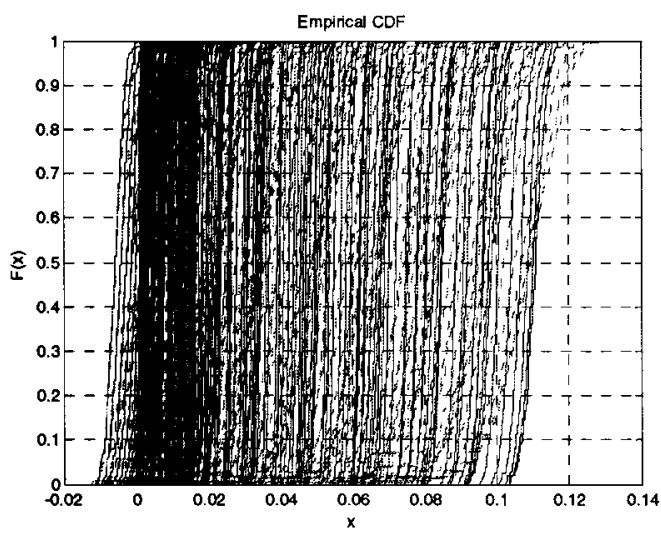

Real part of S21

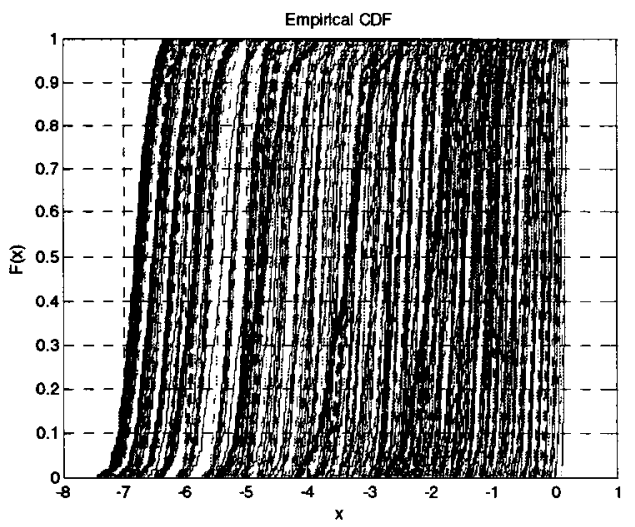

Imaginary part of S11

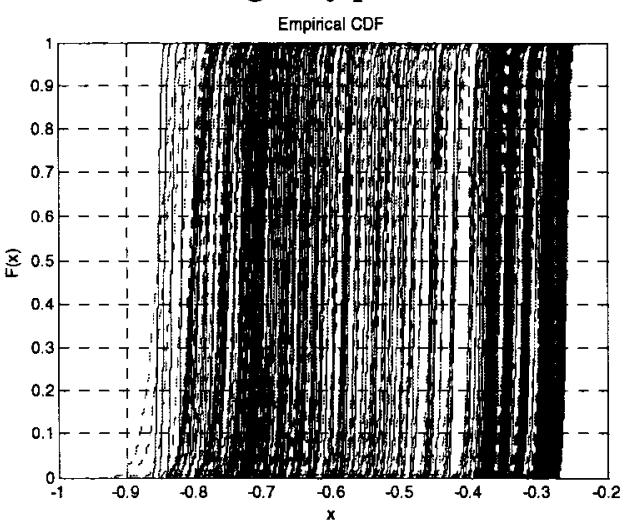

Imaginary part of S12

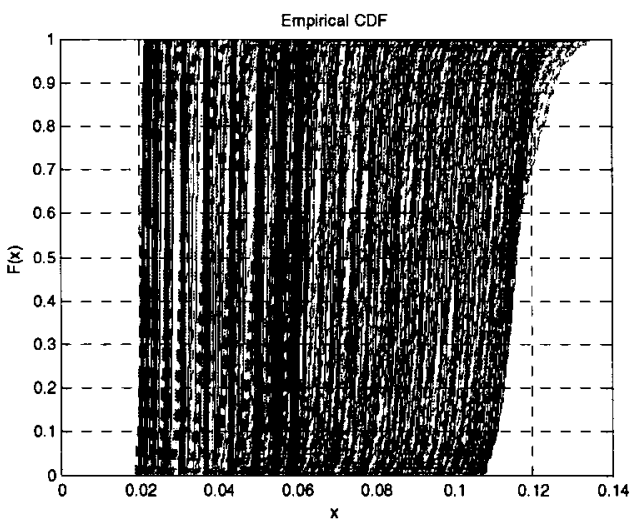

Imaginary part of $S 21$

Empirical CDF

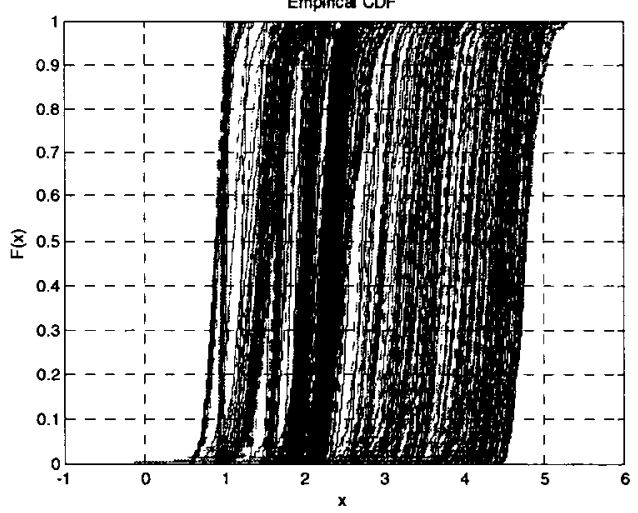

Figure 4.8 Comparison of ECDF of DC and S-parameters in real and imaginary parts between 250 MESFET devices (dash line) and 250 proposed statistical linear dynamic space mapping models (solid line). Overall error is $6.60 \%$. 


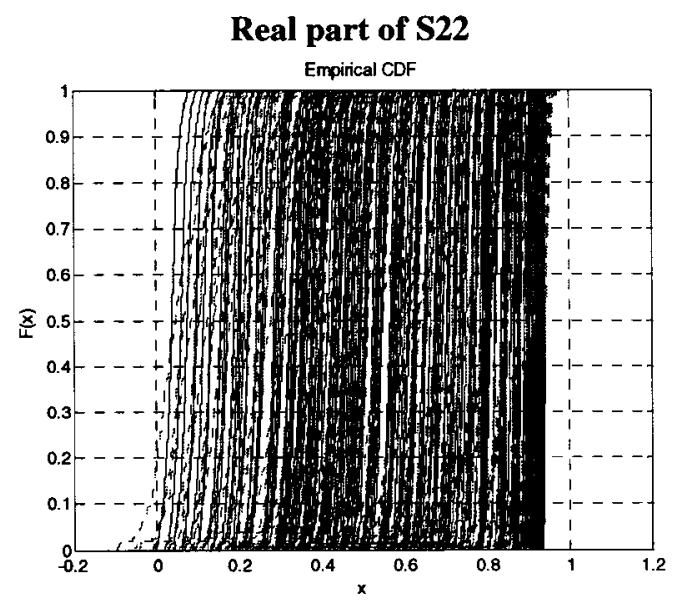

\section{Imaginary part of S22}
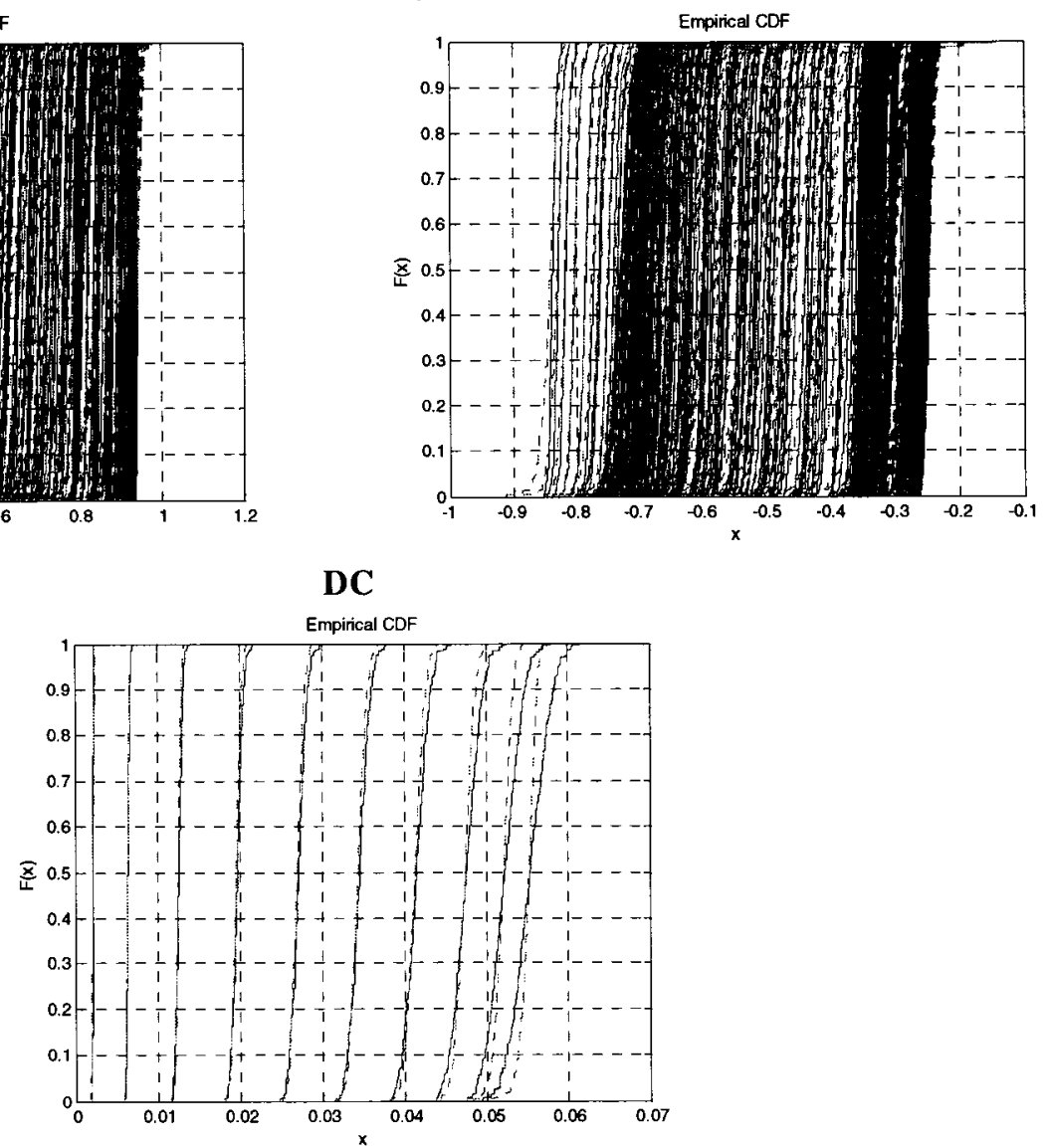

Figure 4.8 (Continued). 

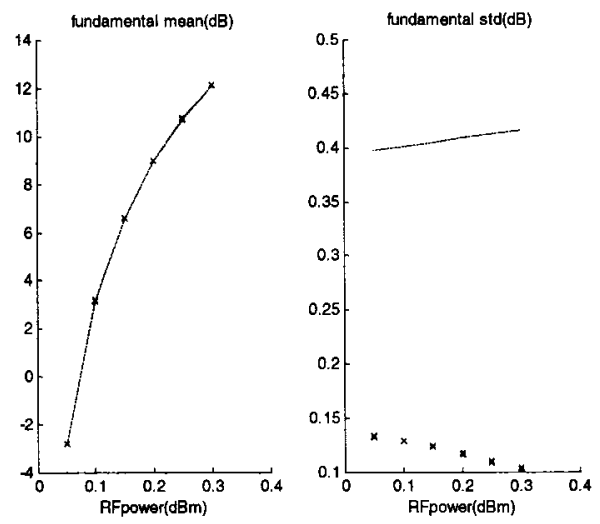

(a)
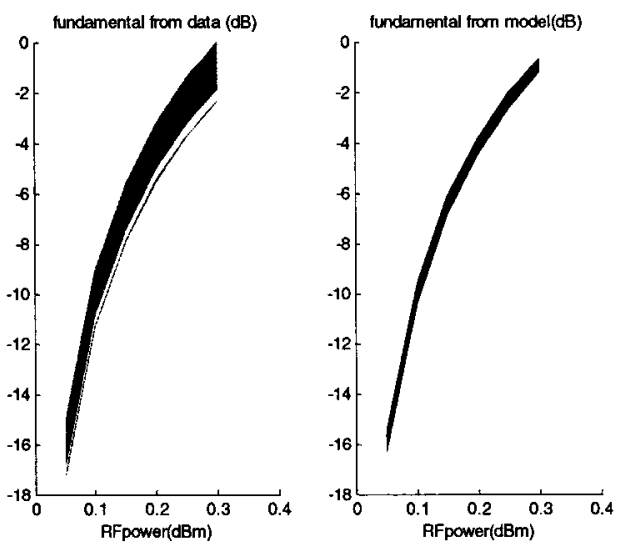

(b)

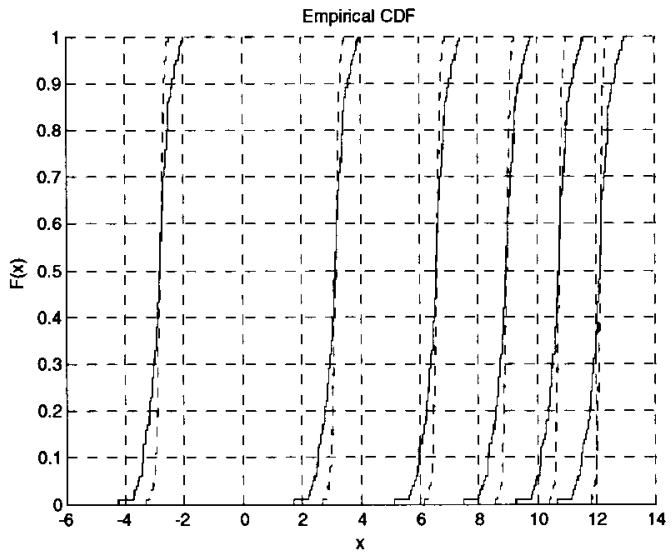

(c)

Figure 4.9 Fundamental harmonic comparisons between 100 statistical models and 100 devices: (a) Mean and standard deviation device in (-) and model in (x). (b) Distribution (c) ECDF - device in dash line and model in solid line. 

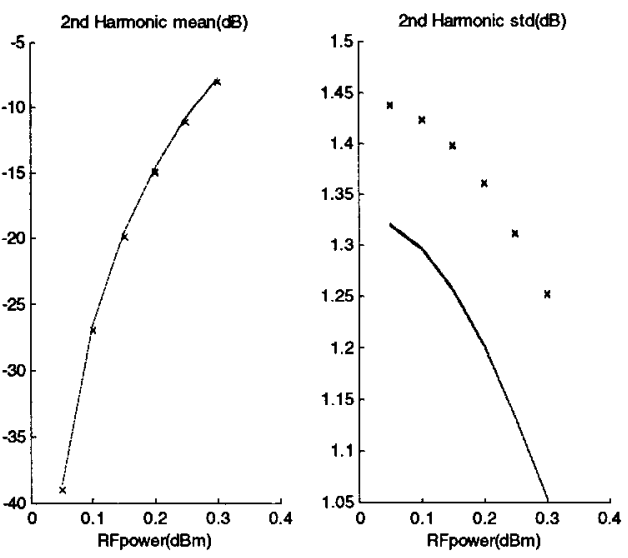

(a)
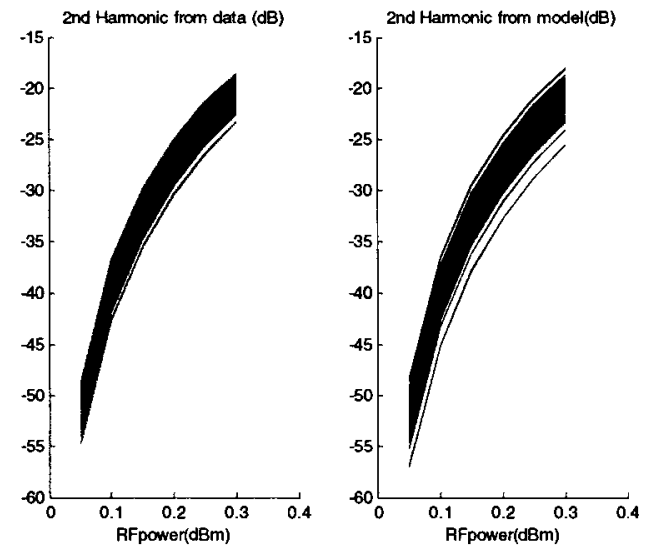

(b)

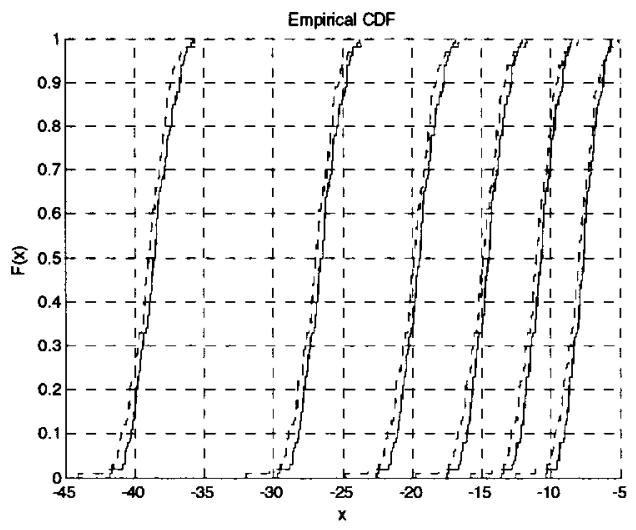

(c)

Figure 4.10 Second harmonic comparisons between 100 statistical models and 100 devices: (a) Mean and standard deviation device in (-) and model in (x). (b)

Distribution (c) ECDF - device in dash line and model in solid line. 

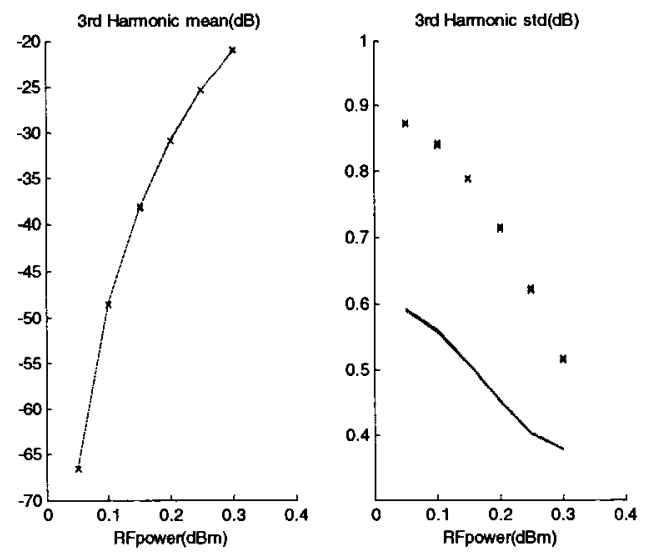

(a)
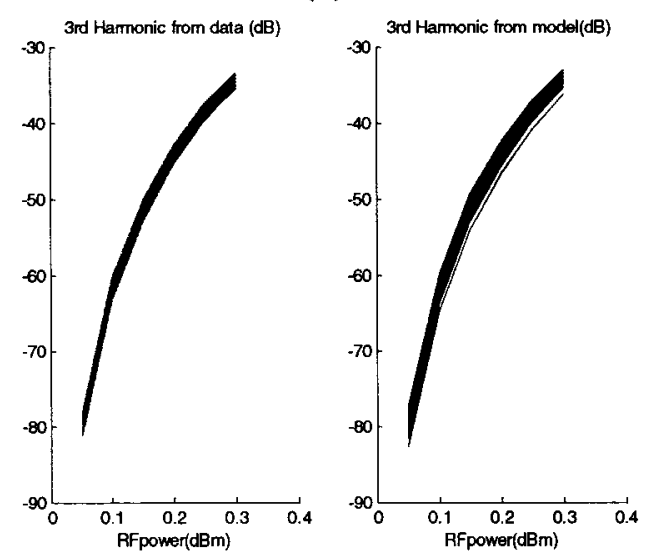

(b)

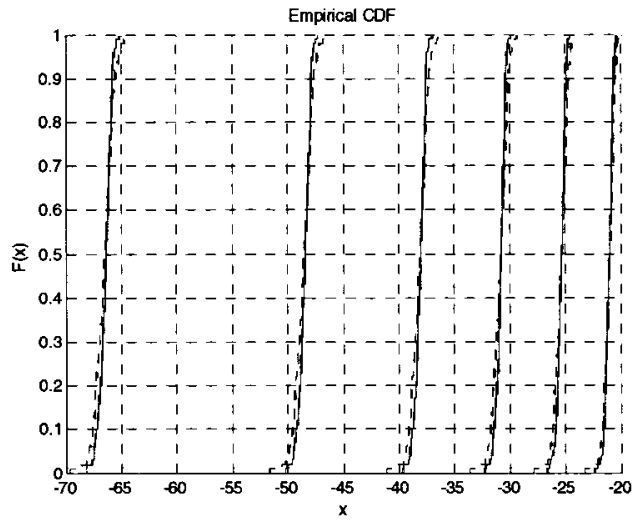

(c)

Figure 4.11 Third harmonic comparisons between 100 statistical models and 100 devices: (a) Mean and standard deviation device in (-) and model in (x). (b) Distribution (c) ECDF - device in dash line and model in solid line. 
Table 4.12

Comparison Between the Device Data and the Statistical Linear Dynamic Mapping Model.

\begin{tabular}{|l|l|l|l|l|l|}
\hline \multicolumn{5}{|c|}{ Linear Dynamic Space Mapping Model Testing Results } \\
\hline $\begin{array}{l}\text { Extraction } \\
\text { accuracy }\end{array}$ & $\begin{array}{l}\text { Order } \\
\text { selection }\end{array}$ & Comparison & $\begin{array}{l}\text { Error of } \\
\text { mean \% }\end{array}$ & $\begin{array}{l}\text { Error of } \\
\text { SD \% }\end{array}$ & $\begin{array}{l}\text { Error of } \\
\text { ECDF \% }\end{array}$ \\
\hline \multirow{3}{*}{$3.0 \%$} & \multirow{2}{*}{10 variables } & DC and S & 1.54 & 47.07 & 4.83 \\
\cline { 3 - 6 } & & HB & 1.60 & 26.11 & 5.48 \\
\hline \multirow{2}{*}{$5.0 \%$} & \multirow{2}{*}{18 variables } & DC and S & 1.64 & 51.02 & 6.60 \\
\cline { 3 - 6 } & & HB & 1.68 & 32.56 & 7.27 \\
\hline \multirow{2}{*}{$10.0 \%$} & \multirow{2}{*}{18 variables } & DC and S & 10.56 & 122.55 & 10.96 \\
\cline { 3 - 6 } & & HB & 27.36 & 220.24 & 25.71 \\
\hline
\end{tabular}

Because the nonlinear property of this HEMT model, it is harder to be modeled. When the device variation is increased to $10 \%$, the proposed technique is no longer able to handle the modeling case since the optimization error is huge. We may try to increase the order, i.e. add more statistical variables; however, it will take longer time to do the optimization and also it is hard to do the analysis of the statistical parameters. This is also because too many parameters may contain redundant ones and too much freedom for them may make the statistical parameters have huge variations, which will lead to a wrong distribution of the statistical parameters.

Although, this example did not show perfect matches in all the three variation cases in Table 4.12, we still see that when the device variation is small enough, the proposed technique is able to do the statistical modeling in large-signal. One harmonic comparison to conventional technique using 100 devices is also performed with the 5\% case, and it used 49 equivalent circuit parameters to achieve ECDF error of $42.13 \%$, which is much larger than $7.27 \%$. This also concludes that the proposed technique is better than the conventional technique by using less statistical parameters. 


\subsection{Statistical Model Used in Amplifier Simulation}

To further demonstrate the proposed technique, we examine the statistical behavior of a population of 50 devices represented by an internal MESFET [5] in ADS. The ADS device parameters are perturbed around given mean values by specified standard deviations. The nominal model in this example is the MESFET model whose parameters are exactly the mean values. The statistical parameters in the space mapping network are extracted from DC and bias-dependent S-parameters of each device in the population for a reasonable extraction accuracy of $1 \%$ error. Each set of DC and S-parameter data is generated at 150 bias points and 20 frequencies. The derivative orders, i.e., $N_{i}$ and $N_{\text {nomi }}(i=1,2)$, used in this example are all equal to one. After parameter extraction, $\mu, \sigma$, and $\rho$ of the parameters $\phi$ are calculated as shown in Tables 4.13 and 4.14. To test the result, the overall statistical model including the nominal model and the statistical space mapping network is then used for large-signal Monte-Carlo analysis with 100 devices. The same analysis is also done to the original MESFET device. The comparisons of the output power and the output current for all the 100 devices are given in Figs. 4.12 and 4.13, showing that the proposed model can catch the large-signal statistical properties of the device. 

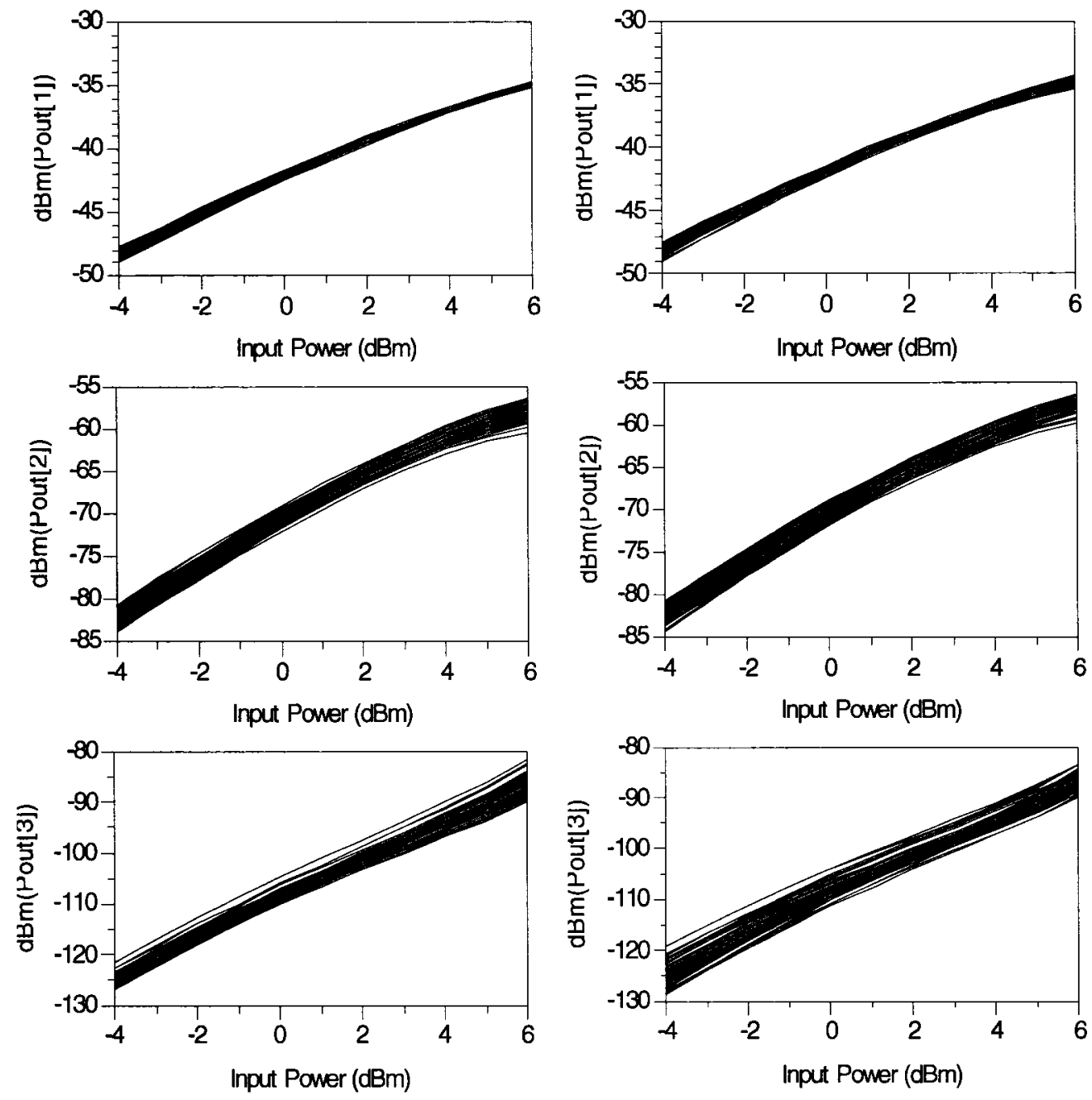

Figure.4.12. Example of output power (fundamental to third harmonics) vs. input power of Monte-Carlo simulations with 100 devices using (a) original ADS MESFET and (b) proposed statistical space-mapped model. 


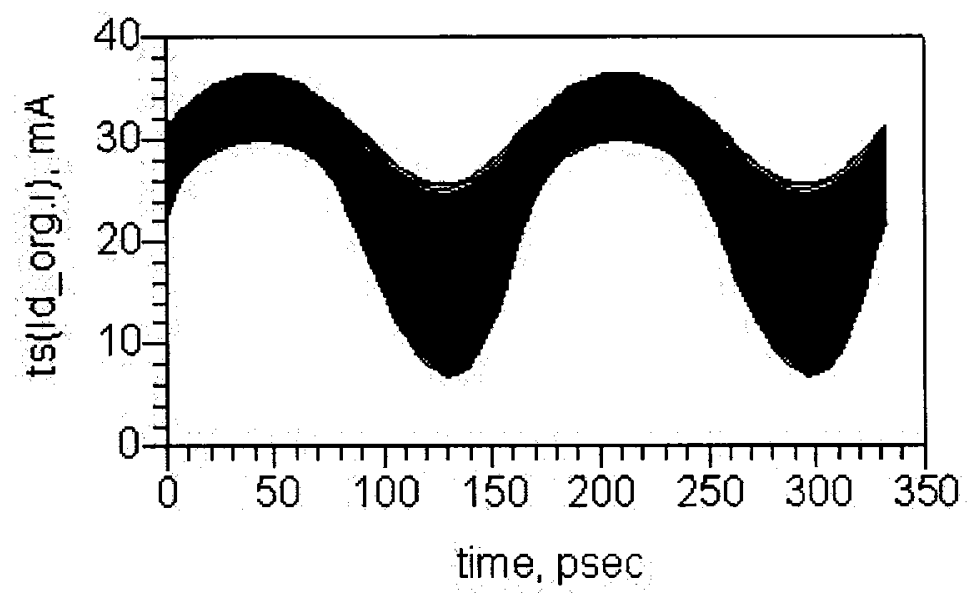

(a)

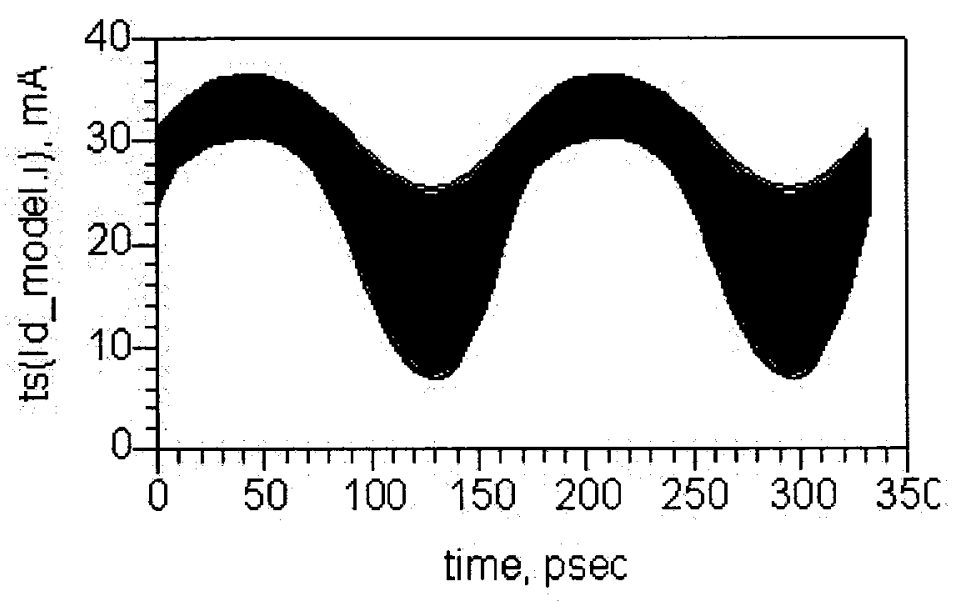

(b)

Figure. 4.13. Example of output current of Monte-Carlo simulations with 100 devices using (a) original ADS MESFET and (b) proposed statistical space-mapped model. 
TABLE 4.13

Means and Standard Deviation of the Statistical Space Mapping Parameters

\begin{tabular}{|c|c|c|}
\hline Parameter $(\phi)$ & Mean $(\mu)$ & Standard Deviation $(\sigma)$ \\
\hline$a_{10}$ & 1.004 & $1.943 \mathrm{e}-2$ \\
\hline$a_{11}$ & $4.940 \mathrm{e}-4$ & $5.106 \mathrm{e}-3$ \\
\hline$b_{10}$ & $-2.968 \mathrm{e}-3$ & $4.921 \mathrm{e}-3$ \\
\hline$c_{11}$ & $-4.912 \mathrm{e}-4$ & $5.095 \mathrm{e}-3$ \\
\hline$d_{l}$ & $4.866 \mathrm{e}-4$ & $5.657 \mathrm{e}-2$ \\
\hline$a_{20}$ & $-2.178 \mathrm{e}-2$ & $1.349 \mathrm{e}-2$ \\
\hline$a_{21}$ & $2.135 \mathrm{e}-3$ & $6.054 \mathrm{e}-3$ \\
\hline$b_{20}$ & 1.059 & $7.872 \mathrm{e}-2$ \\
\hline$b_{21}$ & $-4.315 e-2$ & $8.455 e-2$ \\
\hline$c_{21}$ & $3.838 \mathrm{e}-2$ & $7.181 \mathrm{e}-2$ \\
\hline$d_{2}$ & $-4.912 \mathrm{e}-4$ & $3.297 \mathrm{e}-2$ \\
\hline
\end{tabular}


TABLE 4.14

Correlation Coefficients of the Statistical Space Mapping Parameters

\begin{tabular}{|l|lllllllllll|}
\hline \multicolumn{10}{|c|}{ Correlation Coefficients $(\rho)$} \\
\hline$\phi$ & $a_{10}$ & $a_{11}$ & $b_{10}$ & $c_{11}$ & $d_{1}$ & $a_{20}$ & $a_{21}$ & $b_{20}$ & $b_{21}$ & $c_{21}$ & $d_{2}$ \\
\hline$a_{10}$ & 1.00 & 0 & & & & & & & \\
\hline$a_{11}$ & -0.22 & 1.00 & & & & & & \\
\hline$b_{10}$ & -0.39 & -0.29 & 1.00 & & & & & & \\
\hline$c_{11}$ & -0.30 & -0.86 & 0.48 & 1.00 & & & & & \\
\hline$d_{1}$ & -0.20 & 0.21 & 0.16 & -0.09 & 1.00 & & & & \\
\hline$a_{20}$ & -0.60 & 0.12 & 0.61 & 0.18 & -0.07 & 1.00 & & & \\
\hline$a_{21}$ & -0.18 & 0.26 & -0.43 & -0.14 & -0.41 & 0.09 & 1.00 & & \\
\hline$b_{20}$ & 0.95 & -0.21 & -0.44 & -0.28 & -0.28 & -0.67 & -0.11 & 1.00 & & \\
\hline$b_{21}$ & -0.39 & 0.02 & -0.05 & 0.16 & -0.01 & 0.23 & -0.01 & -0.36 & 1.00 & \\
\hline$c_{21}$ & -0.10 & 0.09 & 0.28 & -0.02 & 0.15 & 0.06 & 0.08 & -0.13 & -0.87 & 1.00 \\
\hline$d_{2}$ & -0.39 & 0.03 & 0.38 & 0.17 & 0.14 & 0.71 & 0.02 & -0.62 & -0.02 & 0.25 & 1.00 \\
\hline
\end{tabular}


To continue to demonstrate the capability of this technique, we use the statistical space-mapped model from this example into a three-stage amplifier simulation as shown in Fig. 4.14. After performing 1000 Monte-Carlo analyses to two amplifier circuits: one uses the original MESFET device in ADS; another uses our proposed statistical model, the yield results of amplifier gain are very close, which are $73.6 \%$ and $68.9 \%$, respectively.

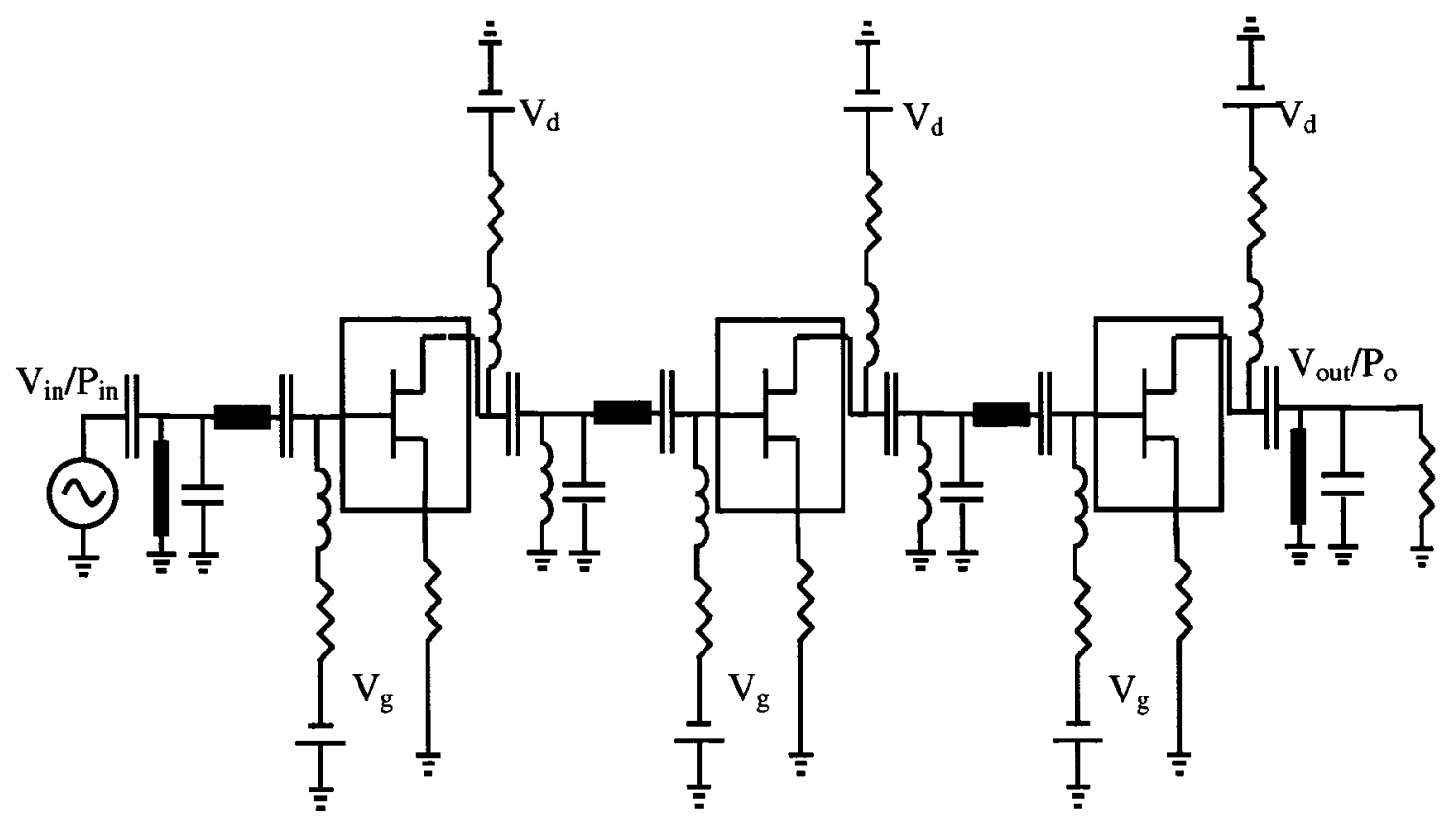

Figure. 4.14 Three-stage amplifier circuit. 


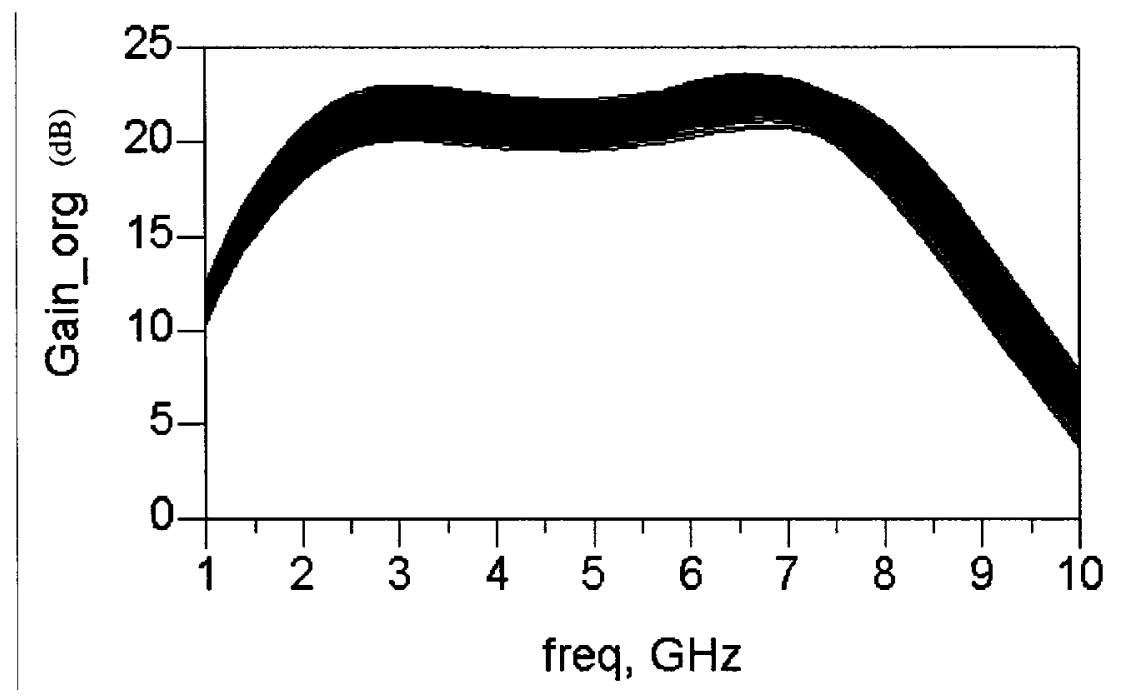

(a)

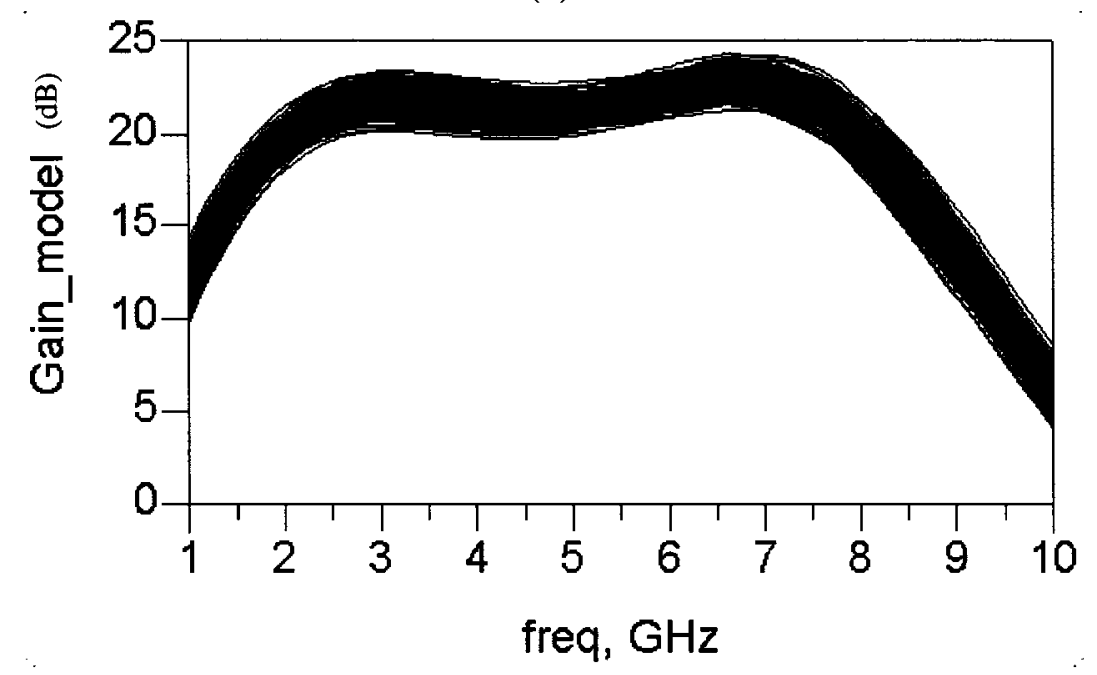

(b)

Figure. 4.15. Gain comparison of 1000 amplifier circuits using (a) original ADS MESFET and (b) proposed statistical space-mapped model. The distribution of the amplifier responses using proposed statistical space-mapped model matches that of the original ADS results well, confirming the proposed method. 
The primary goal of statistical modeling is to provide accurate models for yield simulation. Yield is commonly approximated by the primitive Monte Carlo (PMC) method [15]. The advantage of the PMC analysis is that the method is completely general, with no assumptions regarding circuit complexity or the complexity of the input parameter statistics. The accuracy of the PMC yield estimate, however, for a given confidence is a function of the number of trials used to form that estimate. According to the first and second example, the standard deviation errors are high, but a main reason that will make the errors acceptable. The reason is referred to yield simulation, in other words, it exams how many good results will appear in a certain range. For example, in a population sample of 100 , if the desired mean is 0 and standard deviation is 1 , and the modeled mean is 0.001 and standard deviation is 1.4. This means they have almost perfect match in mean comparison and $40 \%$ error in standard deviation comparison; however, there might be only three samples fall far away from the mean and cause the large variation in the model, when the model is used in yield simulation, these three bad samples will not have large effect on the whole simulation. Fig. 4.15 shows the comparison of the gain of the amplifier circuits in yield simulation and there is only about $5 \%$ difference. This shows that the proposed statistical space-mapped model can be used for statistical design of high-level circuit. 


\section{Chapter 5}

\section{Conclusions and Future Research}

A new approach of large-signal statistical modeling technique has been presented. This technique combines a large-signal nominal model and a set of linear dynamic space mapping functions to characterize the statistical variations around the nominal performance. The nominal model is an accurate large-signal model extracted from a complete large-signal measurement. The mapping functions are used to compensate the difference between device samples and the nominal model, taking statistical inputs extracted from DC and bias-dependent S-parameter data of all device samples. The examples of MESFET, HEMT, and the final yield simulation have shown that the technique is able to accurately catch the large-signal statistical behavior of a device population. The proposed technique can provide accurate large-signal statistical model while reducing the development cost by using only set of large-signal measurement. Thus it is useful for microwave circuit design involving highly repetitive computations such as design optimization, statistical design, and yield optimization.

As a future direction, we will continue to expand the knowledge-based nonlinear device modeling approach into the large-signal statistical modeling and we could still use 
neural networks to represent the variation around the nominal model. Instead of using the mapping neural network weights or linear dynamic coefficients as the statistical parameters, we can use a set of free statistical variables to represent the process variations as the inputs to a mapping neural network. In addition, because the new development in neural networks training algorithm, we now could reduce the training time by using the parallel automatic model generation [57], which means we can now reduce the model development time for the statistical parameter extraction.

After all, the proposed technique for statistical modeling in large-signal has avoided the trial-and-error based manual extraction of large-signal models, and it has also achieved the statistical modeling accuracy by using less statistical parameters comparing to the conventional technique. 


\section{Bibliography}

[1] J. W. Bandler, R. M. Biernacki, S. H. Chen, J. F. Loman, M. L. Renault, and Q. J. Zhang, "Combined discrete/normal statistical modeling of microwave devices," Proc. IEEE $19^{\text {th }}$ Eur. Microwave Conf., London, U.K., pp. 205-210, September 1989.

[2] J. W. Bandler, R. M. Biernacki, Q. Cai, and S. H. Chen, "A novel approach to statistical modeling using cumulative probability distribution fitting," IEEE MTT-S Int. Microwave Symp. Dig., pp. 385-388, 1994.

[3] J. F. Swidzinski, and K. Chang, "Nonlinear statistical modeling and yield estimation technique for use in Monte Carlo simulations", IEEE Trans. Microwave Theory and Tech., vol. 48, no. 12, pp. 2316-2324, Decemeber 2000.

[4] A. D. Martino, P. Marietti, M. Olivieri, P. Tommasino, and A. Trifiletti, "Statistical nonlinear model of MESFET and HEMT devices", IEE Proc. on Circuits Devices Syst., vol. 150, no. 2, pp. 95-103, April 2003.

[5] W. Stiebler, F. Rose, and J. Selin, "Nonlinear statistical modeling of large-signal device behavior", IEEE MTT-S Int. Microwave Symp. Dig., Phoenix, AZ, pp. 20712074, May 2001.

[6] L. Zhang, J. J. Xu, M.C.E. Yagoub, R. T. Ding, and Q. J. Zhang, "Efficient analytical formulation and sensitivity analysis of neuro-space mapping technique for nonlinear microwave device modeling", IEEE Trans. Microwave Theory and Tech., vol. 53, no. 9, pp. 2752-2767, September 2005. 
[7] L. Zhang, K. Bo, Q. J. Zhang, and J. Wood, "Statistical space mapping approach for large-signal nonlinear device modeling," in Proc. IEEE $36^{\text {th }}$ Eur. Microw. Conf., Manchester, U.K., Sep. 2006, pp. 676-679.

[8] H. Statz, P. Newman, I.W. Smith, R.A. Pucel, and H.A. Haus, "GaAs FET device and circuit simulation in SPICE", IEEE Trans. Electron Devices, vol. 34, no. 2, pp. 160-169, February 1987.

[9] H. Zaabab, Q.J Zhang and M.S. Nakhla, "A neural network approach to circuit optimization and statistical design," IEEE Trans. Microwave Theory Tech., vol. 43, pp. 1349-1358, 1995

[10] J.W. Bandler, R.M. Biernacki, Q. Cai, S. H. Chen, S. Ye and Q. J. Zhang, “Integrated physics-oriented statistical modeling, simulation and optimization", IEEE Trans. Microwave Theory Tech., vol. 40, pp. 1374-1400, 1992.

[11] F. Wang and Q.J. Zhang, "Knowledge-based neural models for microwave design", IEEE Trans. Microwave Theory Tech., vol. 45, pp. 2333-2343, 1997.

[12] R. Biernacki, J.W. Bandler, J. Song, and Q.J. Zhang, "Efficient quadratic approximation for statistical design", IEEE Trans. Circuit Syst., vol. 36, pp. 1449$1454,1989$.

[13] P. Meijer, "Fast and smooth highly nonlinear multidimensional table models for device modeling", IEEE Trans. Circuit Syst., vol. 37, pp. 335-346, 1990.

[14] Q.J. Zhang, F. Wang, and M.S. Nakhla, "Optimization of high-speed VLSI interconnects: A review", Int. J. of Microwave and Millimeter-Wave CAD, vol. 7, pp. 83-107, 1997. 
[15] A. Veluswami, M.S. Nakhla, and Q.J. Zhang, "The application of neural networks to EM-based simulation and optimization of interconnects in high-speed VLSI circuits", IEEE Trans. Microwave Theory Tech., vol. 45, pp. 712-723, 1997.

[16] F. Wang and Q.J. Zhang, "Knowledge-based neural models for microwave design", IEEE Trans. Microwave Theory Tech., vol. 45, pp. 2333-2343, 1997.

[17] P.M. Watson and K.C. Gupta, "EM-ANN models for microstrip vias and interconnects in multilayer circuits," IEEE Trans. Microwave Theory Tech., vol. 44, pp. 2495-2503, 1996.

[18] G.L. Creech, B.J. Paul, C.D. Lesniak, T.J. Jenkins, and M.C. Calcatera "Artificial neural networks for fast and accurate EM-CAD of microwave circuits," IEEE Trans. Microwave Theory Tech., vol. 45, pp. 794-802, 1997.

[19] P.M. Watson and K.C. Gupta, "Design and optimization of CPW circuits using EMANN models for CPW components", IEEE Trans. Microwave Theory Tech., vol. 45, pp. 2515-2523, 1997.

[20] P. Burrascano, M. Mongiardo, C. Fancelli, and M. Mongiardo, "A neural network model for CAD and optimization of microwave filters", IEEE Int. Microwave Symp., (Baltimore, MD), pp. 13-16, 1998.

[21] Y. H. Fang, M.C.E. Yagoub, F. Wang and Q.J. Zhang, "A new macromodeling approach for nonlinear microwave circuits based on recurrent neural networks", IEEE Trans. Microwave Theory Tech., vol. 48, pp. 2335-2344, 2000.

[22] C. Christodoulou, A.E. Zooghby, and M. Georgiopoulos, " Neural network processing for adaptive array antennas" IEEE-APS Int. Symp., (Orlando, FL), pp.2584-2587, 1999. 
[23] X. Ding, J.J. Xu, M.C.E. Yagoub, Q.J. Zhang, "A new modeling approach for embedded passives exploiting state space formulation", $32^{\text {th }}$ European Microwave Conf. (EuMC 2002), (Milan, Italy), 2002.

[24] Q.J. Zhang, M.C.E. Yagoub, X. Ding, D. Goulette, R. Sheffield, and H. Feyzbakhsh, "Fast and accurate modeling of embedded passives in multi-layer printed circuits using neural network approach", Elect. Components \& Tech. Conf., (San Diego, CA), pp. 700-703, 2002.

[25] Y. Harhouss, J. Rousset, H. Chéhadé, E. Ngoya, D. Barataud and J.P. Teyssier, "The use of artificial neural networks in nonlinear microwave devices and circuits modeling: an application to telecommunication system design", Int. J. RF and Microwave CAE, Special Issue on Applications of ANN to RF and Microwave Design, vol. 9, pp. 198-215, 1999.

[26] V.B. Litovski, J.I. Radjenovic, Z.M. Mrcarica, and S.L. Milenkovic, "MOS transistor modeling using neural network," Elect. Lett., vol. 28, pp. 1766-1768, 1992.

[27] V.K. Devabhaktuni, C. Xi, and Q.J. Zhang, "A neural network approach to the modeling of heterojunction bipolar transistors from S-parameter data," Proc. $28^{\text {th }}$ European Microwave Conf., (Amsterdam, Netherlands), Oct. 1998, pp. 306-311.

[28] K. Shirakawa, M. Shimizu, N. Okubo and Y. Daido, "Structural determination of multilayered large signal neural network HEMT model”, IEEE Trans. Microwave Theory Tech., vol. 46, pp. 1367-1375, 1998. 
[29] J.W. Bandler, M.A. Ismail, J.E. Rayas-Sánchez, and Q.J. Zhang, "Neuromodeling of microwave circuits exploiting space mapping technology," IEEE Trans. Microwave Theory Tech., vol. 47, pp. 2417-2427, 1999.

[30] P.M. Watson, C. Cho, and K.C. Gupta, "Electromagnetic-artificial neural network model for synthesis of physical dimensions for multilayer asymmetric coupled transmission structures", Int. J. RF and Microwave CAE, Special Issue on Applications of ANN to RF and Microwave Design, vol. 9, pp. 175-186, 1999.

[31] M. Vai and S. Prasad, "Neural networks in microwave circuit design - beyond black box models", Int. J. RF and Microwave CAE, Special Issue on Applications of ANN to RF and Microwave Design, vol. 9, pp. 187-197, 1999.

[32] X. Ding, B. Chattaraj, M.C.E. Yagoub, V.K. Devabhaktuni, Q.J. Zhang, "EM based statistical design of microwave circuits using neural models", Int. Symp. on Microwave and Optical Technology (ISMOT 2001), (Montreal, Quebec), pp.421426, 2001. (Invited Paper).

[33] Q.J. Zhang and K.C. Gupta, Neural Networks for RF and Microwave Design, Norwood, MA: Artech House, 2000.

[34] C. Cybenko, "Approximation by superpositions of a sigmoidal function," Math. Control Signals Systems, vol. 2, pp. 303-314, 1989.

[35] K. Hornik, M. Stinchombe, and H. White, "Multilayer feedforward networks are universal approximators," Neural Networks, vol. 2, pp. 359-366, 1989.

[36] R. Reed, "Pruning algorithms - a survey," IEEE Trans. Neural Networks, vol. 4, pp. 740-747, September 1993. 
[37] V.K. Devabhaktuni, M.C.E. Yagoub and Q.J. Zhang, "A robust algorithm for automatic development of neural network models for microwave applications," IEEE Trans. Microwave Theory Tech., vol. 49, pp. 2282-2291.

[38] J. de Villiers and E. Barnard, "Backpropagation neural nets with one and two hidden layers," IEEE Trans. Neural Networks, vol. 4, pp. 136-141, 1992.

[39] Q. Li and R. W. Dutton, "Numerical small-signal AC modelling of deep-level-trap related frequency dependent output conductance and capacitance for GaAs MESFET's on semi-insulating substrates", IEEE Trans. Electron Devices, vol. 38, pp. 1285-1288, 1991.

[40] M. A. Khatibzadeh and R. J. Trew, "A large-signal analytical model for the GaAs MESFET”, IEEE Trans Microwave Theory Tech., vol. 36, pp. 231-238, 1988.

[41] C.M. Snowden and R. R. Pantoja, "Quasi two-dimensional MESFET simulation for CAD”, IEEE Trans. Electron Devices, vol. 36, pp. 1564-1574, 1989.

[42] V. Devabhaktuni, M.C.E. Yagoub, Y. Fang, J.J. Xu and Q.J. Zhang, "Neural networks for microwave modeling: model development issues and nonlinear techniques," Int. J. RF and Microwave CAE, vol. 11, pp. 4-21, 2001. (invited review paper).

[43] F. Wang and Q.J. Zhang, "Knowledge-based neural models for microwave design", IEEE Trans. Microwave Theory Tech., vol. 45, pp. 2333-2343, 1997.

[44] S. Goasguen, S.M. Hammadi, and S.M. El-Ghazaly, "A global modeling approach using artificial neural network", IEEE MTT-S Int. Microwave Symp. Digest, (Anaheim, CA), pp.153-156, 1999. 
[45] J.J Xu, M.C.E. Yagoub, and Q.J. Zhang, "Neural based dynamic modeling of nonlinear microwave circuits" IEEE MTT-S Int. Microwave Symp. Digest, (Seattle, WA), pp. 1101-1104, 2002.

[46] J.W. Bandler, M.A. Ismail, J.E. Rayas-Sanchez, and Q.J. Zhang, "Neural inverse space mapping EM optimization," IEEE MTT-S International Microwave Symp. Digest, (Phoenix, AZ), vol. 2, pp. 1007-1010, 2001.

[47] V.K. Devabhaktuni, B. Chattaraj, M. Yagoub and Q.J. Zhang, “Advanced microwave modeling framework exploiting automatic model generation, knowledge based neural networks and space mapping," IEEE MTT-S International Microwave Symp. Digest, (Seattle, WA), pp. 1097-1100, 2002.

[48] J.J Xu, M.C.E. Yagoub, and Q.J. Zhang, "Exact adjoint sensitivity for neural based microwave modeling and design," IEEE MTT-S Int. Microwave Symp. Digest, (Phoenix, AZ), pp. 1015-1018, 2001.

[49] A. D. Martino, P. Marietti, M. Olivieri, P. Tommasino, and A. Trifiletti, "Statistical nonlinear model of MESFET and HEMT devices", IEE Proc. on Circuits Devices Syst., vol. 150, no. 2, pp. 95-103, April 2003.

[50] W. R. Curtice, "GaAs MESFET modeling and nonlinear CAD", IEEE Trans. Microw. Theory Tech., vol. 36, no. 2, pp. 220-230, Feb. 1988.

[51] M. S. Nakhla and J. Vlach, "A piecewise harmonic balance technique for determination of periodic response of nonlinear systems", IEEE Trans. Circuits and Syst., vol. 23, no. 2, pp. 85-91, Feb. 
[52] Y. Cao, R. T. Ding, and Q. J. Zhang, "State-space dynamic neural network technique for high-speed IC applications: modeling and stability analysis," IEEE Trans. Microw. Theory Tech., vol. 54, no. 6, pp. 2398-2409, June 2006

[53] ADS ver. 2006A, Agilent Technologies, Palo Alto, CA, 2006.

[54] Medici ver. Z-2007.03-0, Synopsys., Mountain View, CA, 2007

[55] Agilent Angelov (Chalmers) Nonlinear GaAsFET Model, ADS2006A Manual, "Nonlinear Devices”, Chapter 3.

[56] IC-CAP ver. 2006B, Agilent Technologies, Palo Alto, CA, 2006.

[57] L Zhang, Y. Cao, and Q.J. Zhang, " Parallel Automatic Model Generation Technique for Microwave Modeling,"," IEEE MTT-S Int. Microwave Symp. Dig., Honolulu, Hawaii, June 2007. 